\title{
Gradings on the Albert algebra and on $\mathfrak{f}_{4}$
}

\section{Cristina Draper Fontanals and Cándido Martín González}

\begin{abstract}
We study group gradings on the Albert algebra and on the exceptional simple Lie algebra $\mathfrak{f}_{4}$ over algebraically closed fields of characteristic zero. The immediate precedent of this work is [13] where we described (up to equivalence) all the gradings on the exceptional simple Lie algebra $\mathfrak{g}_{2}$. In the cases of the Albert algebra and $\mathfrak{f}_{4}$, we look for the nontoral gradings finding that there are only eight nontoral nonequivalent gradings on the Albert algebra (three of them being fine) and nine on $\mathfrak{f}_{4}$ (also three of them fine).
\end{abstract}

\section{Introduction}

The interest on gradings on simple Lie and Jordan algebras has been remarkable in the last years. The gradings of finite dimensional simple Lie algebras, ruling out $\mathfrak{a}_{l}, \mathfrak{d}_{4}$ and the exceptional cases, are described in [7]. The gradings on simple Jordan algebras of type $H_{n}(F)$ and $H_{n}(Q)$ are given in the same reference, for an algebraically closed field $F$ of characteristic zero and a quaternion algebra $Q$. In that work, the authors use their previous results in [9] about gradings of associative algebras $M_{n}(F)$. In [6] all gradings on the simple Jordan algebras of Clifford type have been described. The fine gradings on $\mathfrak{a}_{l}$ have been determined in [21] solving the related problem of finding maximal abelian groups of diagonalizable automorphisms of the algebras (not only in $\operatorname{GL}(n, \mathbb{C})$ but also in $\mathrm{O}(n, \mathbb{C})$ for $n \neq 8$ and $\operatorname{SP}(2 n, \mathbb{C})$ ). General notions about Lie gradings are considered in [37], and the real case is treated in [22]. Notice that all the mentioned works make use of techniques related to the associative case. The first studies of gradings on exceptional

2000 Mathematics Subject Classification: Primary: 17B25; Secondary: 17C40.

Keywords: Exceptional Lie algebra, grading, algebraic group, Weyl group. 
Lie algebras are [13] and [8], which describe the group gradings on $\mathfrak{g}_{2}$. To continue the study of exceptional Lie and Jordan algebras, we tackle in this paper the task of describing nontoral group gradings on $\mathfrak{f}_{4}$ and on the Albert algebra. The notion of group grading is closely related to that of commuting set of semisimple automorphisms (or equivalently abelian subgroup of semisimple automorphisms) of the algebra. But $F_{4}$, the automorphism group of the Albert algebra $J$, is isomorphic to the automorphism group of the Lie algebra $\mathfrak{f}_{4}=\operatorname{Der}(J)$. So automorphism information can be transferred from one to the other context. That is the reason to study both algebras jointly.

We have ruled out the study of toral gradings on $\mathfrak{f}_{4}$ (and then on $J$ ) because of the overwhelming proliferation of nonequivalent cases, and the fact that, their determination (though tedious) follows from mechanical coarsenings of the Cartan grading. On the contrary, the nontoral gradings on a simple Lie algebra are not compatible with the root system. So they could lead us to new ways of looking at the algebra. This is specially true in the case of fine gradings, a fact that explains the activity around this subject (see, for instance, [23] or [35]). In other papers of Lie gradings ([36]), the aim is to study the Lie gradings without the restriction of a grading group, but we have preferred not to adopt this approach because of [16]. It is still an open question the existence of a grading group on any finite dimensional graded simple Lie algebra over an algebraically closed field of characteristic zero.

The techniques used to search the gradings have been of very different nature. It could be said that this is a multidisciplinary field nowadays. There is a first tool, very intuitive, which is the usage of models of the algebra. Although it provides most of the existing gradings (actually all of them), this fact is not easy to prove without a more powerful tool. This is why we exploit the benefit of working inside the normalizer of a maximal torus of the automorphism group. This turns out to be quite technical and less intuitive (some computer aided arguments have been essential). However this approach allows to confirm with full precision all the hypothesis about how many nontoral gradings appear, the relation among them and other aspects.

A summary of the contents of the work follows in the next paragraphs.

Section 2 presents some preliminaries, and compiles basic facts on gradings and related topics. In Subsection 2.1 we introduce some terminology of algebraic groups needed for our approach. In 2.2 we devote some attention to recall the most known model of the Albert algebra, $J=H_{3}(C)$ for a Cayley algebra $C$, described in [39]. Each model of a given algebra has its 
own advantages (and drawbacks) to present particular gradings, thus in 2.4 Tits construction of the Albert algebra is also recalled. In 2.3 we consider the notion of toral grading. In the Lie algebras case, a grading is toral when its homogeneous components are sum of root spaces. In general a grading is toral if it is produced by a set of automorphisms contained in a torus of the automorphism group. At this point we fix a maximal torus of $F_{4}$, and characterize the torality of a grading in different terms.

In Section 3 we start inducing gradings on $J$ from gradings on the related Cayley algebra $C$ such that $J=H_{3}(C)$. This arises from the well-known possibility of extending automorphisms of $C$ to elements in $F_{4}\left(G_{2} \subset F_{4}\right)$. It turns out that the unique (up to equivalence) nontoral grading of $\mathfrak{g}_{2}$ induces a nontoral grading on $\mathfrak{f}_{4}$ (a $\mathbb{Z}_{2}^{3}$-grading). Thus we are led to the birth of our first nontoral grading on $J$. This grading will induce a numerous family of gradings whose relatives come from a mixing process described in Subsection 3.3. Previously, we consider all the gradings of the subalgebra $H_{3}(F)$, where $F$ stands for the ground field. But automorphisms of $H_{3}(F)$ can also be nicely extended to automorphisms of $J$. And curiously all of these commute with automorphisms of $J$ coming from $C$. Thus by crossing the nontoral $\mathbb{Z}_{2}^{3}$-grading on $J$ coming from $C$ with all the gradings detected in $H_{3}(F)$ we obtain a family of six nontoral gradings on $J$ described in 3.3. But, as it is pointed out at the end of such subsection, one of these six nontoral gradings admits a proper nontoral coarsening. Thus we are led to a set of seven nonequivalent nontoral gradings on $J$.

Unfortunately we are not done with this set of gradings. To detect the remaining nontoral gradings on $J$ we need to invoke some other model of $J$ different from the usual $H_{3}(C)$. But at this point, Tits construction comes in our help to provide a $\mathbb{Z}_{3}^{3}$-grading with comes from the natural embedding of automorphisms of the algebra $M_{3}(F)$ into $F_{4}$. The origin of this $\mathbb{Z}_{3}^{3}$ grading is a known nontoral $\mathbb{Z}_{3}^{2}$-grading of $M_{3}(F)$ which can be lifted and finally refined to the nontoral $\mathbb{Z}_{3}^{3}$-grading on $J$. In this way we get a set of eight pairwise nonequivalent nontoral gradings. Furthermore, any nontoral grading is equivalent to some of these. This is one of our main results presented at the end of Section 3 (though the proof will have to be postponed to the final sections of the work).

In Section 4 we focus on $\mathfrak{f}_{4}$. How can we present a concrete grading on a Lie algebra? One of the ways in which a grading on an algebra can be given is to provide the set of automorphisms inducing the grading. Specially because the automorphisms can be given in terms of toral elements and the Weyl group. This happens because of the relevant fact that the quasitorus inducing the grading is always contained in the normalizer of a maximal torus. Consequently, our first task is to fix a particular representation of 
its Weyl group $\mathcal{W}$ and provide a set of representatives of conjugacy classes in $\mathcal{W}$. Next we present in Subsection 4.1 a maximal torus of $\mathfrak{F}_{4}:=\operatorname{aut}\left(\mathfrak{f}_{4}\right)$ nicely related to the maximal torus previously presented in $F_{4}:=\operatorname{aut}(J)$. Also the action of $\mathcal{W}$ on the maximal torus is described.

Section 5 is intended to provide the main results on quasitori which will allow the classification of fine and nontoral gradings. We introduce a family of quasitori $A(j, t) \subset \mathfrak{F}_{4}$ and immediately proceed with the study of those which are nontoral. Theorem 4 describes the maximal quasitori of $\mathfrak{F}_{4}$ up to conjugacy which in particular yields the classification of fine gradings on $\mathfrak{f}_{4}$ up to equivalence (Corollary 4). Finally in Subsection 5.2 we get a more detailed classification up to conjugacy of nontoral quasitori of $\mathfrak{F}_{4}$. This result contained in Theorem 5 might be of independent interest. As a byproduct, we get an exhaustive set of representatives of 9 equivalence classes of nontoral gradings on $\mathfrak{f}_{4}$.

In Section 6 we revisit the gradings on the Albert algebra to provide a proof that, up to equivalence, the nontoral gradings on this algebra are the eight ones given in Theorem 3.

Having given the quasitori which induce all the nontoral gradings on $\mathfrak{f}_{4}$ we look more closely at the fine gradings in Section 7 . There, we describe those gradings in a twofold way. First we give the homogeneous components of each grading in a previously fixed basis of the algebra. This description depends on computational methods (simultaneous diagonalization algorithms). In spite of their computational nature, these descriptions may be interesting for applications in which explicit calculations are needed. The second description procedure for the gradings is the exhibition of models, which make the grading appear in a natural setting with no appeal to a particular basis. For instance, the fine $\mathbb{Z}_{3}^{3}$-grading on $\mathfrak{f}_{4}$ comes from a $\mathbb{Z}_{3}^{3}$-grading in $\mathfrak{e}_{6}$ which can be described in a very convenient way by using the known model based in the $\mathbb{Z}_{3}$-grading

$$
\mathfrak{e}_{6}=\operatorname{sl}\left(X_{1}\right) \oplus \operatorname{sl}\left(X_{2}\right) \oplus \operatorname{sl}\left(X_{3}\right) \oplus X_{1} \otimes X_{2} \otimes X_{3} \oplus X_{1}^{*} \otimes X_{2}^{*} \otimes X_{3}^{*}
$$

with zero homogeneous component three copies of the algebra of type $\mathfrak{a}_{2}$ $\left[1\right.$, p. 85]. The fine $\mathbb{Z}_{2}^{5}$-grading may be seen directly in $\mathfrak{f}_{4}=\operatorname{Der}(J)$ but there is also an easy way to see it in the Tits unified construction for the exceptional Lie algebras (see, for instance, [39, p. 122]). The same applies to the $\mathbb{Z}_{2}^{3} \times \mathbb{Z}$-fine grading. Finally, in 7.4 we justify why this philosophy can be valid for describing most of the gradings on a simple Lie algebra. To illustrate how this works, we describe the $\mathbb{Z}_{2}^{2} \times \mathbb{Z}_{4}$ and $\mathbb{Z}_{2}^{2} \times \mathbb{Z}_{8}$-nontoral gradings on $\mathfrak{f}_{4}$, and also the $\mathbb{Z}_{2}^{3} \times \mathbb{Z}_{8}$-fine grading equivalent to the $\mathbb{Z}_{2}^{3} \times \mathbb{Z}$ one, by using the model of $\mathfrak{f}_{4}$ described in [12], which is based in an initial $\mathbb{Z}_{4}$-grading. 


\section{Preliminary definitions and results}

\subsection{Group gradings}

Our aim is the study of group gradings on certain nonassociative algebras over fields. If $V$ is such an algebra and $G$ is an abelian group, we shall say that the decomposition

$$
V=\bigoplus_{g \in G} V_{g}
$$

is a $G$-grading whenever for all $g, h \in G, V_{g} V_{h} \subset V_{g h}$.

In this work we shall use some notions borrowed from the theory of algebraic groups. Since we only need linear algebraic groups, all concepts must be understood in that context. Notice that the group of automorphisms of the algebra $V$ is an algebraic linear group. The ground field $F$ will be supposed to be algebraically closed and of characteristic zero throughout this work. There is a deep relationship between gradings on $V$ and quasitori of the group of automorphisms aut $(V)$, according to [34, §3, p. 104]. Following this reference, a commutative algebraic group whose identity component is an algebraic torus is called an algebraic quasitorus. An algebraic linear group is a quasitorus if and only if there is a basis relative to which the elements of the quasitorus are simultaneously diagonalizable. If $S$ is a finitely generated abelian group, then its group of characters $\mathfrak{X}(S)=\operatorname{hom}\left(S, F^{\times}\right)$is a quasitorus and reciprocally, the group of characters of a quasitorus turns out to be a finitely generated abelian group.

If $V=\oplus_{g \in G} V_{g}$ is a $G$-grading, the map $\psi: \mathfrak{X}(G) \rightarrow \operatorname{aut}(V)$ mapping each $\alpha \in \mathfrak{X}(G)$ to the automorphism $\psi_{\alpha}: V \rightarrow V$ given by $V_{g} \ni x \mapsto \psi_{\alpha}(x):=$ $\alpha(g) x$ is a group homomorphism. In particular $\psi(\mathfrak{X}(G))$ is a quasitorus. And conversely, if $Q$ is a quasitorus and $\psi: Q \rightarrow$ aut $(V)$ is a homomorphism, $\psi(Q)$ is formed by semisimple automorphisms ([26, p. 99]) and we have a $\mathfrak{X}(Q)$-grading $V=\oplus_{g \in \mathfrak{X}(Q)} V_{g}$ given by

$$
V_{g}=\{x \in V \mid \psi(q)(x)=g(q) x \forall q \in Q\} .
$$

When we speak in this paper about a $G$-grading $V=\oplus_{g \in G} V_{g}$, we mean that $G$ is generated by the set $\left\{g \in G \mid V_{g} \neq 0\right\}$, called the support of the grading and denoted by $\operatorname{Supp}(G)$. In terms of algebraic groups, this is equivalent to the fact that the homomorphism $\psi: Q \rightarrow$ $\operatorname{aut}(V)$ is injective. Let us see it. If $G$ is generated by the support and $\psi\left(q_{0}\right)=\operatorname{id}_{V}$ for some $q_{0} \in Q, x=\psi\left(q_{0}\right) x=g\left(q_{0}\right) x$ for any $x \in V_{g}$, so $g\left(q_{0}\right)=1$ for any $g$ in the support. But since $G$ is generated by the support, we have $g\left(q_{0}\right)=1$ for any $g \in G$ and this implies $q_{0}=1$. 
Reciprocally if $\psi$ is a monomorphism, the subgroup $S$ of $G$ generated by the support is $S=\mathfrak{X}\left(Q^{\prime}\right)$ for some quasitori $Q^{\prime}$. The inclusion $i: S \rightarrow G$ induces by duality an epimorphism $\pi: Q \rightarrow Q^{\prime}$. The grading induced by $S$ comes from a homomorphism $\psi^{\prime}: Q^{\prime} \rightarrow$ aut $(V)$ mak-

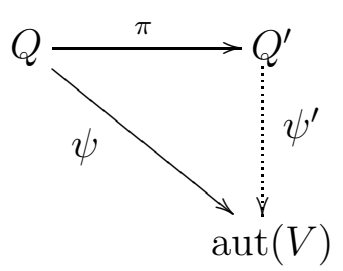
ing commutative the diagram on the right. Since $\psi$ is a monomorphism, so is $\pi$. Hence $\pi$ is an isomorphism and by duality the same can be said about $i$. Thus $S=G$.

We say that two gradings $V=\oplus_{g \in G} X_{g}=\oplus_{g^{\prime} \in G^{\prime}} Y_{g^{\prime}}$ are isomorphic if there is $f \in \operatorname{aut}(V)$ and $\alpha: G \rightarrow G^{\prime}$ a group isomorphism such that $f\left(X_{g}\right)=$ $Y_{\alpha(g)}$ for any $g \in G$. So, if $\psi: \mathfrak{X}(G) \rightarrow$ aut $(V)$ and $\psi^{\prime}: \mathfrak{X}\left(G^{\prime}\right) \rightarrow \operatorname{aut}(V)$ are the corresponding homomorphisms, and we take $\alpha^{*}: \mathfrak{X}\left(G^{\prime}\right) \rightarrow \mathfrak{X}(G), \alpha^{*}(\beta)=$ $\beta \alpha$, and $\operatorname{Ad}(f): \operatorname{aut}(V) \rightarrow \operatorname{aut}(V)$ given by $\operatorname{Ad}(f)(\rho)=f \rho f^{-1}$, the previous condition is equivalent to the commutativity $\operatorname{Ad}(f) \psi^{\prime}=\psi \alpha^{*}$.

We say that two gradings $V=\oplus_{g \in G} X_{g}=\oplus_{g^{\prime} \in G^{\prime}} Y_{g^{\prime}}$ are equivalent if the sets of homogeneous subspaces are the same up to isomorphism, that is, there are an automorphism $f \in \operatorname{aut}(V)$ and a bijection between the supports $\alpha: \operatorname{Supp}(G) \rightarrow \operatorname{Supp}\left(G^{\prime}\right)$ such that $f\left(X_{g}\right)=Y_{\alpha(g)}$ for any $g \in \operatorname{Supp}(G)$. Our objective is to classify gradings up to equivalence. A convenient invariant for equivalence is that of type. Suppose we have a grading on a finite dimensional algebra, then for each positive integer $i$ we will denote (following [24]) by $h_{i}$ the number of homogeneous components of dimension $i$. In this case we shall say that the grading is of type $\left(h_{1}, h_{2}, \ldots, h_{l}\right)$, for $l$ the greatest index such that $h_{l} \neq 0$. Of course the number $\sum_{i} i h_{i}$ agrees with the dimension of the algebra.

Another key notion is that of a coarsening of a given grading. Thus consider an $F$-algebra $V$, a $G$-grading $V=\oplus_{g \in G} X_{g}$ and an $H$-grading $V=$ $\oplus_{h \in H} Y_{h}$. We shall say that the $H$-grading is a coarsening of the $G$-grading if and only if each nonzero homogeneous component $Y_{h}$ with $h \in H$ is a direct sum of some homogeneous components $X_{g}$. In this case we shall also say that the $G$-grading is a refinement of the $H$-grading. Notice that there is not a relationship between $G$ and $H$, but if $\psi: \mathfrak{X}(G) \rightarrow \operatorname{aut}(V)$ and $\psi^{\prime}: \mathfrak{X}(H) \rightarrow \operatorname{aut}(V)$ are the homomorphisms producing the above gradings, any automorphism in $\psi^{\prime}(\mathfrak{X}(H))$ acts as a scalar multiple of the identity in $X_{g}$ and hence, all of them commute with $\psi(\mathfrak{X}(G))$.

The concept of universal grading group is fundamental to obtain the coarsenings of a given grading. Though this notion is given in the context of simple Lie algebras in [13, Section 2.2], it can be translated to more general settings. Consider as before a nonassociative $F$-algebra $V$, but with a grading $V=\oplus_{i \in I} V_{i}$ which is not supposed to be a group grading, that is, 
each $V_{i}$ is nonzero and for any $i, j \in I$ there is $k \in I$ such that $V_{i} V_{j} \subset V_{k}$. Define the group $G_{I}:=\mathbb{Z}(I) / M$ where $\mathbb{Z}(I)$ is the free $\mathbb{Z}$-module generated by $I$ and $M$ the $\mathbb{Z}$-submodule generated by the elements $i+j-k$ such that $0 \neq V_{i} V_{j} \subset V_{k}$. We shall denote by $\bar{x}$ the equivalence class of $x \in \mathbb{Z}(I)$ in the quotient $G_{I}$. We get a $G_{I^{-}}$grading on $V$ given by $V=\oplus_{\bar{i} \in G_{I}} V_{\bar{i}}^{\prime}$ where $V_{\bar{i}}^{\prime}=\sum_{\bar{j}=\bar{i}} V_{j}$. In general this new grading is not equivalent to the original one. For instance we could have $G_{I}=0$, in which case the new grading is the trivial one. But if the original grading of $V$ is equivalent to an abelian group grading, then it is easily proved that the canonical map $I \rightarrow G_{I}$ given by $i \mapsto \bar{i}$ is injective and the new grading is equivalent to the original one. Moreover any coarsening of this grading comes from a group epimorphism as in [13, Proposition 2(2), p.90]. More precisely, if $V=\oplus_{g \in G} X_{g}$ is a $G$-grading and $V=\oplus_{h \in H} Y_{h}$ is a coarsening, then if $G$ is the universal grading group, there is an epimorphism $p: G \rightarrow H$ such that $Y_{h}=\oplus_{p(g)=h} X_{g}$. It is this universal property which suggests the term universal when applied to $G_{I}$. Getting back to our context, (abelian) group gradings, we can characterize the universal grading group in terms of linear algebraic groups. If $\psi: Q \rightarrow$ aut $(V)$ is a grading with $G=\mathfrak{X}(Q)$, then $G$ is the universal grading group if and only if for any coarsening $\psi^{\prime}: Q^{\prime} \rightarrow \operatorname{aut}(V)$, there is a monomorphism $i: Q^{\prime} \rightarrow Q$ such that $\psi^{\prime}=\psi i$. This is easily proved applying duality to the above universal property of $G$. From another viewpoint, $G$ is the universal group of a grading if and only if $\mathfrak{X}(G)$ is a maximal element in the set of quasitori of aut $(V)$ producing exactly the same grading.

A group grading is fine if its unique refinement is the given grading. If $G$ is the universal group of a grading $\psi: \mathfrak{X}(G) \rightarrow$ aut $(V)$, the grading is fine if and only if $\psi(\mathfrak{X}(G))$ is a maximal abelian subgroup of semisimple elements, which is usually called a $M A D$ ("maximal abelian diagonalizable") in papers about fine gradings, like [20]. Besides, each MAD $Q \subset \operatorname{aut}(V)$ produces a $\mathfrak{X}(Q)$-fine grading on $V$ such that $\mathfrak{X}(Q)$ is the universal group of this grading. The converse is true in the sense just mentioned but notice that there are fine $G$-gradings such that $\psi(\mathfrak{X}(G))$ is not a MAD, for instance the $\mathbb{Z}_{2}^{3} \times \mathbb{Z}_{8}$-grading on $\mathfrak{f}_{4}$ described in 7.4 or the $\mathbb{Z}$-grading on $\mathfrak{g}_{2}$ described in [13, Theorem 2,(4)]. In particular from the above, the number of fine gradings on $V$ up to equivalence is the same than the number of MAD's of aut $(V)$ up to conjugation (and less than the number of fine gradings up to isomorphism, in general).

Other notations which will be used along this paper are the following. For a linear algebraic group $G$ and a subset $S \subset G$, the centralizer of $S$ in $G$ will be denoted by $\mathfrak{C}_{G}(S)$. Analogously, by $\mathfrak{N}_{G}(S)$ we shall mean the normalizer of $S$ in $G$. 


\subsection{About the Albert algebra and $\mathfrak{f}_{4}$}

Consider the Cayley $F$-algebra $C$, which under our hypothesis on the ground field must be isomorphic to Zorn's vector matrix algebra. Take the standard involution $x \mapsto \bar{x}$, the norm $n: C \rightarrow F$ given by $n(x):=x \bar{x}$ and the trace $\operatorname{tr}: C \rightarrow F$ defined as $\operatorname{tr}(x):=x+\bar{x}$. Recall that the polar form of $n$ is the symmetric bilinear form $b_{n}: C \times C \rightarrow F$ such that $b_{n}(x, y):=\frac{1}{2}(n(x+y)-$ $n(x)-n(y))$. The Albert algebra

$$
J=H_{3}(C)=\left\{x=\left(x_{i j}\right) \in M_{3}(C) \mid x_{i j}=\overline{x_{j i}}\right\}
$$

is the exceptional reduced Jordan algebra, that is, the set of matrices of the form

$$
\left(\begin{array}{lll}
\alpha_{1} & o_{3} & \overline{o_{2}} \\
\overline{o_{3}} & \alpha_{2} & o_{1} \\
o_{2} & \overline{o_{1}} & \alpha_{3}
\end{array}\right)
$$

where $\alpha_{i} \in F$ and $o_{i} \in C(i=1,2,3)$. Since our base field is of characteristic zero, we can shelter on the linear theory of Jordan algebras and the product in $J$ may be defined as $x \cdot y:=\frac{1}{2}(x y+y x)$, where juxtaposition stands for the usual matrix product. This simple Jordan algebra is exceptional in the sense that it is not a subalgebra of the symmetrization of any associative algebra. It will be convenient to introduce some notations for further reference. Thus the element in $J$ obtained in (2.1) for $\alpha_{i}=1$ and $\alpha_{j}=o_{k}=0$ for $j \neq i$ and any $k$ will be denoted by $E_{i}$, so that $1:=\sum_{1}^{3} E_{i}$ is the unit of $J$. And for any $a \in C$ we define by $a^{(i)}$ the element in $J$ obtained making $o_{i}=a$ and $o_{j}=\alpha_{k}=0$ for $j \neq i$ and any $k$, in (2.1). The multiplication table of the commutative algebra $J$ may be summarized in the following relations:

$$
\begin{array}{lll}
E_{i}^{2}=E_{i}, & E_{i} \cdot a^{(i)}=0, & a^{(i)} \cdot b^{(i)}=b_{n}(a, b)\left(E_{j}+E_{k}\right), \\
E_{i} \cdot E_{j}=0, & E_{i} \cdot a^{(j)}=\frac{1}{2} a^{(j)}, & a^{(i)} \cdot b^{(j)}=\frac{1}{2}(\bar{b} \bar{a})^{(k)},
\end{array}
$$

where $(i, j, k)$ is any cyclic permutation of $(1,2,3)$ and $a, b \in C$. Following Schafer $\left(\left[39,(4.41)\right.\right.$, p. 109]), any $x \in J$ satisfies a cubic equation $x^{3}-$ $\operatorname{Tr}(x) x^{2}+\mathrm{Q}(x) x-\mathrm{N}(x) 1=0$ where $\operatorname{Tr}(x), \mathrm{Q}(x), \mathrm{N}(x) \in F$. Moreover, the inversibility of $x$ (in Jordan context) is equivalent to the fact that $N(x) \neq 0$.

For further reference we fix first $\left(e_{1}, e_{2}, u_{1}, u_{2}, u_{3}, v_{1}, v_{2}, v_{3}\right)$ the standard basis of the Cayley algebra $C$, defined for instance in [13, Section 3], given by the relations

$$
\begin{array}{lll}
e_{1} u_{j}=u_{j}=u_{j} e_{2}, & u_{i} u_{j}=v_{k}=-u_{j} u_{i}, & u_{i} v_{i}=e_{1}, \\
e_{2} v_{j}=v_{j}=v_{j} e_{1}, & -v_{i} v_{j}=u_{k}=v_{j} v_{i}, & v_{i} u_{i}=e_{2},
\end{array}
$$

where $e_{1}$ and $e_{2}$ are orthogonal idempotents, again $(i, j, k)$ is any cyclic permutation of $(1,2,3)$, and the remaining relations are null. Thus we can fix 
our standard basis of the Albert algebra:

$$
\begin{gathered}
B=\left(E_{1}, E_{2}, E_{3}, e_{1}^{(3)}, e_{2}^{(3)}, u_{1}^{(3)}, u_{2}^{(3)}, u_{3}^{(3)}, v_{1}^{(3)}, v_{2}^{(3)}, v_{3}^{(3)}, e_{2}^{(2)}, e_{1}^{(2)},-u_{1}^{(2)},-u_{2}^{(2)},\right. \\
\left.-u_{3}^{(2)},-v_{1}^{(2)},-v_{2}^{(2)},-v_{3}^{(2)}, e_{1}^{(1)}, e_{2}^{(1)}, u_{1}^{(1)}, u_{2}^{(1)}, u_{3}^{(1)}, v_{1}^{(1)}, v_{2}^{(1)}, v_{3}^{(1)}\right) .
\end{gathered}
$$

Let us define now the group $F_{4}:=\operatorname{aut}(J)$ and its Lie algebra $\mathfrak{f}_{4}=\operatorname{Der}(J)$. Recall (see for instance [27, p. 285]) that the automorphism group $F_{4}$ and the automorphism group $\mathfrak{F}_{4}:=\operatorname{aut}\left(\mathfrak{f}_{4}\right)$ are isomorphic via the map Ad: $F_{4} \rightarrow \mathfrak{F}_{4}$ such that $\operatorname{Ad}(f) d:=f d f^{-1}$ for any $f \in F_{4}$ and $d \in \mathfrak{f}_{4}$. This isomorphism of algebraic groups provides a tool for translating gradings from the Albert algebra to $\mathfrak{f}_{4}$ and conversely. However, unfortunately this translating tool does not preserve equivalence. This phenomenon is similar to the one explained in [13, Section 4] in the similar context produced by the analogue group isomorphism Ad: $G_{2}=\operatorname{aut}(C) \rightarrow \operatorname{aut}\left(\mathfrak{g}_{2}\right)=\operatorname{aut}(\operatorname{Der}(C))$.

\subsection{Maximal torus of $F_{4}$}

Let us fix certain maximal torus of $F_{4}$. We use the standard basis of $J$ above defined. Define now the maximal torus $\mathfrak{T}_{0}$ of $F_{4}$ whose elements are the automorphisms of $J$ which are diagonal relative to $B$. This is isomorphic to $\left(F^{\times}\right)^{4}$ and it is easy to see that the matrix of any such automorphism relative to $B$ is

$$
\begin{array}{r}
\operatorname{diag}\left(1,1,1, \alpha, \frac{1}{\alpha}, \beta, \gamma, \frac{\delta^{2}}{\alpha \beta \gamma}, \frac{1}{\beta}, \frac{1}{\gamma}, \frac{\alpha \beta \gamma}{\delta^{2}}, \delta, \frac{1}{\delta}, \frac{\alpha \beta}{\delta}, \frac{\alpha \gamma}{\delta}, \frac{\delta}{\beta \gamma}, \frac{\delta}{\alpha \beta}, \frac{\delta}{\alpha \gamma}, \frac{\beta \gamma}{\delta},\right. \\
\left.\frac{\delta}{\alpha}, \frac{\alpha}{\delta}, \frac{\beta}{\delta}, \frac{\gamma}{\delta}, \frac{\delta}{\alpha \beta \gamma}, \frac{\delta}{\beta}, \frac{\delta}{\gamma}, \frac{\alpha \beta \gamma}{\delta}\right)
\end{array}
$$

for some $\alpha, \beta, \gamma, \delta \in F^{\times}$. Define now $t_{\alpha, \beta, \gamma, \delta}$ as the automorphism in $\mathfrak{T}_{0}$ whose matrix relative to $B$ is just the above one.

Consider a grading of an algebra $A$ given by a group homomorphism $\rho: \mathfrak{X}(G) \rightarrow \operatorname{aut}(A)$. This grading is said to be toral if $\rho(\mathfrak{X}(G))$ is contained in some torus of the algebraic group aut $(A)$.

Notice that by means of the above isomorphism Ad: $F_{4} \rightarrow \mathfrak{F}_{4}$, a grading on $J$ is toral if and only if it is applied to a toral grading on $\mathfrak{f}_{4}$. This does not imply that the number of nontoral gradings (up to equivalence) on $J$ and on $\mathfrak{f}_{4}$ is necessarily the same, because the mechanism of translating gradings does not preserve equivalence.

An useful characterization of the torality of a grading on $J$ is the following:

Proposition 1. A grading on the Albert algebra $J$ is toral if and only if the elements of the standard basis of $J$ (or any conjugated basis) are homogeneous. 
Proof. Consider a toral grading induced by automorphisms $\left\{t_{i}\right\}_{i \in I}$ all of them contained in the previous maximal torus $\mathfrak{T}_{0}$ of $J$ (which supposes no restriction because any other maximal tori is conjugated to $\left.\mathfrak{T}_{0}\right)$. Then obviously the simultaneous diagonalization of $J$ relative to the family $\left\{t_{i}\right\}_{i \in I}$ provides the original grading and the elements in the standard basis are homogeneous. Conversely, if this holds, then any element in this basis is an eigenvector of any of the grading automorphisms. Thus these automorphisms are in the maximal torus specified before and the grading is toral.

In particular, the proof of this proposition implies that if a grading on $J$ is toral, then there are three orthogonal idempotents contained in the zero component (because up to conjugacy, $\left\{E_{1}, E_{2}, E_{3}\right\}$ is contained in such component). Another way of checking the torality is to look at the rank of the zero part of the induced grading on $\mathfrak{f}_{4}$, it will be toral in case this rank is 4 (see [13, Subsection 2.4]).

By using this set of idempotents, we can give more information about the general form of any semisimple automorphism of $J$, because they are the building blocks of the grading sets. First notice that there is a group monomorphism $D_{4} \rightarrow F_{4}$. Indeed, if

$$
U \in \mathrm{O}(C, n)=\left\{g \in \mathrm{GL}(C) \mid b_{n}(x, y)=b_{n}(g(x), g(y)) \forall x, y \in C\right\},
$$

there are $U^{\prime}, U^{\prime \prime} \in \mathrm{O}(C, n)$ such that $U(x y)=U^{\prime}(x) U^{\prime \prime}(y)$ for any $x, y \in C$. This is called global triality principle in [11, Th.3, p.90]. Let $\Psi_{U}: J \rightarrow J$ given by

$$
\Psi_{U}\left(E_{i}\right)=E_{i}, \Psi_{U}\left(x^{(i)}\right)=\left(f_{i}(x)\right)^{(i)} \text { for } f_{1}(x)=\overline{U(\bar{x})}, f_{2}=U^{\prime}, f_{3}=U^{\prime \prime} .
$$

It is easy to check that $\Psi_{U}$ is an automorphism of $J$ fixing each idempotent. Conversely, if $\psi$ is an automorphism of $J$ fixing each idempotent, there exists $U \in \mathrm{O}(C, n)$ such that $\psi=\Psi_{U}$. Moreover, any semisimple automorphism in $F_{4}$ is toral, since $F_{4}$ is a connected group, so that up to conjugacy it is contained in $\mathfrak{T}_{0}$, fixes each $E_{i}$ and is of the form $\Psi_{U}$ for some $U \in \mathrm{O}(C, n)$.

Besides, there are two outstanding automorphisms which deserve some consideration. The first one $\theta: J \rightarrow J$ applies $E_{i} \mapsto E_{i+1}$ and $x^{(i)} \mapsto x^{(i+1)}$ cyclically. The second one $\vartheta: J \rightarrow J$ is given by

$$
E_{1} \leftrightarrow E_{2}, \quad E_{3} \mapsto E_{3}, \quad x^{(1)} \leftrightarrow \bar{x}^{(2)}, \quad x^{(3)} \mapsto \bar{x}^{(3)} .
$$

These automorphisms fix the set $\left\{E_{1}, E_{2}, E_{3}\right\}$ and are of order three and two respectively. Our previous argument shows that any semisimple element in $F_{4}$ fixing the set $\left\{E_{1}, E_{2}, E_{3}\right\}$ is a composition of one automorphism in $\left\{\right.$ id, $\left.\theta, \theta^{2}, \vartheta, \vartheta \theta, \vartheta \theta^{2}\right\}$ with one in $\left\{\Psi_{U} \mid U \in \mathrm{O}(C, n)\right\}$. 


\subsection{Tits construction of the Albert algebras}

There is another way in which the Albert algebra can be constructed. Let us start with the $F$-algebra $A=M_{3}(F)$ and denote by $\operatorname{Tr}_{A}, \mathrm{Q}_{A}, \mathrm{~N}_{A}: A \rightarrow F$ the coefficients of the generic minimal polynomial such that

$$
x^{3}-\operatorname{Tr}_{A}(x) x^{2}+\mathrm{Q}_{A}(x) x-\mathrm{N}_{A}(x) 1=0 \quad \text { for all } x \in A .
$$

Recall that if $x=\left(x_{i j}\right) \in A$ then $\operatorname{Tr}_{A}(x)=\sum_{1}^{3} x_{i i}$,

$$
\mathrm{Q}_{A}(x)=-x_{12} x_{21}+x_{11} x_{22}-x_{13} x_{31}-x_{23} x_{32}+x_{11} x_{33}+x_{22} x_{33},
$$

and $\mathrm{N}_{A}(x)=\operatorname{det}(x)$. Define also the quadratic map $\sharp: A \rightarrow A$ given by

$$
x^{\sharp}:=x^{2}-\operatorname{Tr}_{A}(x) x+Q_{A}(x) 1 .
$$

For any $x, y \in A$ denote

$$
x \times y:=(x+y)^{\sharp}-x^{\sharp}-y^{\sharp},
$$

and

$$
x^{*}:=\frac{1}{2} x \times 1=\frac{1}{2} \operatorname{Tr}_{A}(x) 1-\frac{1}{2} x .
$$

Finally consider the Jordan algebra $A^{+}$whose underlying vector space agrees with that of $A$ but whose product is $x \cdot y=\frac{1}{2}(x y+y x)$. Next, define in $A^{3}:=A \times A \times A$ the product

$$
\begin{aligned}
& \quad\left(a_{1}, b_{1}, c_{1}\right)\left(a_{2}, b_{2}, c_{2}\right):= \\
& \left(a_{1} \cdot a_{2}+\left(b_{1} c_{2}\right)^{*}+\left(b_{2} c_{1}\right)^{*}, a_{1}^{*} b_{2}+a_{2}^{*} b_{1}+\frac{1}{2}\left(c_{1} \times c_{2}\right), c_{2} a_{1}^{*}+c_{1} a_{2}^{*}+\frac{1}{2}\left(b_{1} \times b_{2}\right)\right) .
\end{aligned}
$$

Then $A^{3}$ with this product is isomorphic to $J=H_{3}(C)$. This is the so called Tits construction of the Albert algebra. This allows us to identify $J$ with the algebra $A^{3}$ in the rest of this section. For further reference we shall recall that the norm $N$ (module the identification of $J$ with the Tits construction) is given by

$$
\mathrm{N}(a, b, c)=\mathrm{N}_{A}(a)+\mathrm{N}_{A}(b)+\mathrm{N}_{A}(c)-\operatorname{Tr}_{A}(a b c)
$$

for any $a, b, c \in A$ (see [30, p. 525]).

One of the relevant facts on Tits construction from our viewpoint is that it allows to embed aut $(A)$ in $F_{4}$ via the map aut $(A) \rightarrow F_{4}$ given by $f \mapsto f^{\bullet}$ where $f^{\bullet}: J \rightarrow J$ is the automorphism such that $f^{\bullet}(x, y, z):=$ $(f(x), f(y), f(z))$. As a further consequence we will be able to get gradings on $J$ coming from gradings in the associative algebra $A$ via this monomorphism of algebraic groups. 


\section{Inducing gradings on the Albert algebra}

Some remarkable subalgebras of $J=H_{3}(C)$ induce gradings on $J$ by means of a particular embedding of its automorphism group in $F_{4}$. For instance, the automorphism group of $H_{3}(F)$ can be considered as a subgroup of $F_{4}$ providing thus a source of gradings on $J$ : those whose grading automorphisms come from automorphisms of $H_{3}(F)$. More generally, if $V$ is an algebra such that $\operatorname{aut}(V)$ is a subgroup of $F_{4}$, then any grading in $V$ will also induce a grading on $J$. We shall see that this happens for instance for the octonion $F$-algebra. It turns out that this idea for inducing gradings on $J$ provides a great number of the gradings existing on $J$.

\subsection{Gradings from octonions}

Let $C$ be the Cayley algebra. We consider as in the previous section the standard basis of $C$. We shall also need the maximal torus of $G_{2}:=\operatorname{aut}(C)$ given by the automorphisms $t_{\alpha, \beta}$ whose matrix relative to the standard basis is

$$
\operatorname{diag}\left(1,1, \alpha, \beta,(\alpha \beta)^{-1}, \alpha^{-1}, \beta^{-1}, \alpha \beta\right) .
$$

It has been first proved in [15], and then in [13, Subsection 3.3], that up to equivalence the unique nontoral grading on $C$ is the $\mathbb{Z}_{2}^{3}$-grading whose order-two grading automorphisms are $\left\{t_{1,-1}, t_{-1,1}, f_{0}\right\}$ where $f_{0}$ is the automorphism whose matrix relative to the standard basis is

$$
\left(\begin{array}{cccccccc}
0 & 1 & 0 & 0 & 0 & 0 & 0 & 0 \\
1 & 0 & 0 & 0 & 0 & 0 & 0 & 0 \\
0 & 0 & 0 & 0 & 0 & 1 & 0 & 0 \\
0 & 0 & 0 & 0 & 0 & 0 & 1 & 0 \\
0 & 0 & 0 & 0 & 0 & 0 & 0 & -1 \\
0 & 0 & 1 & 0 & 0 & 0 & 0 & 0 \\
0 & 0 & 0 & 1 & 0 & 0 & 0 & 0 \\
0 & 0 & 0 & 0 & -1 & 0 & 0 & 0
\end{array}\right) .
$$

The construction $J=H_{3}(C)$ of the Albert algebra is particularly interesting for extending derivations and automorphisms from $C$ to $J$. More precisely if $f \in \operatorname{aut}(C)$ is an automorphism of $C$ then we can construct the automorphism $\hat{f}$ of $J$ fixing the idempotents $E_{i}$ and such that $\hat{f}\left(o^{(i)}\right):=f(o)^{(i)}$ for $i=1,2,3$ and any $o \in C$. This provides a monomorphism of algebraic groups $i: G_{2} \rightarrow F_{4}$ such that $f \mapsto \hat{f}$. By differentiating at 1 we get a monomorphism of Lie algebras $\operatorname{d} i(1): \operatorname{Der}(C)=\mathfrak{g}_{2} \rightarrow \operatorname{Der}(J)=\mathfrak{f}_{4}$ mapping each derivation $d \in \operatorname{Der}(C)$ to the derivation $\hat{d} \in \operatorname{Der}(J)$ annihilating the idempotents and making $\hat{d}\left(o^{(i)}\right)=d(o)^{(i)}$, for $i=1,2,3$. This, of course, has an immediate application to gradings: any grading on $C$ induces a 
grading on $J$. Indeed, a $G$-grading on $C$ comes from an algebraic group homomorphism $\rho: \mathfrak{X}(G) \rightarrow \operatorname{aut}(C)$, therefore $i \rho: \mathfrak{X}(G) \rightarrow \operatorname{aut}(J)$ provides a $G$-grading on the Albert algebra. This device gives a first source of gradings on $J$, namely, all those coming from gradings on $C$. Since $i$ maps tori of aut $(C)$ to tori of aut $(J)$, toral gradings on $C$ induce toral gradings on $J$. The unique nontoral grading on $C$ up to equivalence provides a $\mathbb{Z}_{2}^{3}$-grading on $J$ induced by the automorphisms $\left\{\widehat{t_{1,-1}}, \widehat{t_{-1,1}}, \widehat{f_{0}}\right\}$ whose homogeneous spaces are

$$
\begin{aligned}
J_{1,1,1} & =\left\langle E_{1}, E_{2}, E_{3}, 1^{(3)}, 1^{(2)}, 1^{(1)}\right\rangle, \\
J_{1,1,-1} & =\left\langle\left(-e_{1}+e_{2}\right)^{(3)},\left(-e_{1}+e_{2}\right)^{(2)},\left(-e_{1}+e_{2}\right)^{(1)}\right\rangle, \\
J_{1,-1,1} & =\left\langle\left(u_{2}+v_{2}\right)^{(3)},\left(u_{2}+v_{2}\right)^{(2)},\left(u_{2}+v_{2}\right)^{(1)}\right\rangle, \\
J_{-1,1,1} & =\left\langle\left(u_{1}+v_{1}\right)^{(3)},\left(u_{1}+v_{1}\right)^{(2)},\left(u_{1}+v_{1}\right)^{(1)}\right\rangle, \\
J_{1,-1,-1} & =\left\langle\left(-u_{2}+v_{2}\right)^{(3)},\left(-u_{2}+v_{2}\right)^{(2)},\left(-u_{2}+v_{2}\right)^{(1)}\right\rangle, \\
J_{-1,1,-1} & =\left\langle\left(-u_{1}+v_{1}\right)^{(3)},\left(-u_{1}+v_{1}\right)^{(2)},\left(-u_{1}+v_{1}\right)^{(1)}\right\rangle, \\
J_{-1,-1,1} & =\left\langle\left(-u_{3}+v_{3}\right)^{(3)},\left(-u_{3}+v_{3}\right)^{(2)},\left(-u_{3}+v_{3}\right)^{(1)}\right\rangle, \\
J_{-1,-1,-1} & =\left\langle\left(u_{3}+v_{3}\right)^{(3)},\left(u_{3}+v_{3}\right)^{(2)},\left(u_{3}+v_{3}\right)^{(1)}\right\rangle .
\end{aligned}
$$

For this grading we have $h_{i}=0$ except $h_{3}=7, h_{6}=1$. Thus the grading is of type $(0,0,7,0,0,1)$. It is easy to prove that the subalgebra of $\mathfrak{f}_{4}$ whose elements are those $d \in \mathfrak{f}_{4}$ such that $\left[d, \widehat{t_{1,-1}}\right]=\left[d, \widehat{t_{-1,1}}\right]=\left[d, \widehat{f_{0}}\right]=0$ is threedimensional. Thus, the grading on $\mathfrak{f}_{4}$ induced by $\left\{\operatorname{Ad}\left(\widehat{t_{1,-1}}\right), \operatorname{Ad}\left(\widehat{t_{-1,1}}\right), \operatorname{Ad}\left(\widehat{f_{0}}\right)\right\}$ is nontoral since its zero homogeneous component has dimension 3 , hence its rank is less than $4=\operatorname{rank}\left(\mathfrak{f}_{4}\right)$. We summarize the results in this subsection in the following:

Proposition 2. The unique nontoral grading on $J$ coming from a grading on $C$ is the above grading (3.1) up to equivalence.

\subsection{Gradings on $H_{3}(F)$}

It is known from [28, p. 184-185] that any automorphism of $H_{3}(F)$ is of the form $\operatorname{In}(p): x \mapsto p x p^{-1}$ where $p$ can be taken in the group $\mathrm{SO}(3):=\mathrm{SO}(3, F)$ consisting of those $3 \times 3$ matrices $x$ with entries in $F$ such that $x x^{t}=1$ and $\operatorname{det}(x)=1$ (the notation $x^{t}$ meaning the transpose of the matrix $x$ ). Thus we have an algebraic group isomorphism $\mathrm{In}: \mathrm{SO}(3) \rightarrow \operatorname{aut}\left(H_{3}(F)\right)=: G$. Now consider the algebra $M_{3}(C)$ with the usual matrix product. This contains, as a subalgebra, $M_{3}(F)$ and for any $x, y, z \in M_{3}(C)$ we have $(x y) z=x(y z)$ if any of $x, y, z$ belongs to $M_{3}(F)$. Since $H_{3}(F)$ is a (Jordan) subalgebra of $J=H_{3}(C)$, for any $p \in \mathrm{SO}(3)$ the map $\operatorname{In}(p): J \rightarrow J$ such that $x \mapsto p x p^{-1}$ (products in $M_{3}(C)$ ) is an automorphism of $J$. Thus we have an algebraic group monomorphism In: $\mathrm{SO}(3) \rightarrow F_{4}$ and thus a monomorphism $\mathrm{SO}(3) \cong$ $G \rightarrow F_{4}=\operatorname{aut}(J)$ so that we can extend automorphisms from $H_{3}(F)$ to $J$ 
(in fact this monomorphism maps $\operatorname{In}(p)$ seen as an element in $G$ to $\operatorname{In}(p)$ as element in $F_{4}$ ). So we have constructed a tool for translating gradings from $H_{3}(F)$ to $J$. This suggests the convenience of describing group gradings on $H_{3}(F)$. The work [7] contains an exhaustive and deep description of gradings on some simple Jordan and Lie algebras, in particular the gradings on $H_{n}(F)$ could be obtained along the line of this work. But in our particular setting it is worth to find this description by using elementary algebraic group theory. This will provide a comfortable landscape for testing geometric tools and will provide a more self contained exposition.

It is well known that a maximal torus $P$ of $\mathrm{SO}(3)$ is given by the matrices of the form

$$
p_{\alpha, \beta}:=\left(\begin{array}{ccc}
1 & 0 & 0 \\
0 & \alpha & \beta \\
0 & -\beta & \alpha
\end{array}\right)
$$

with $\alpha, \beta \in F$ such that $\alpha^{2}+\beta^{2}=1$. Then denote by $\tau_{\alpha, \beta}:=\operatorname{In}\left(p_{\alpha, \beta}\right)$ the corresponding element in $G=\operatorname{aut}\left(H_{3}(F)\right)$. The set of all $\tau_{\alpha, \beta}$ is a maximal torus $\mathcal{T}$ of $G$ and the set of eigenvalues of $\tau_{\alpha, \beta}$ is $S_{\alpha, \beta}=\left\{1, z, z^{-1}, z^{2}, z^{-2}\right\}$ for $z:=\alpha+i \beta$ (where $i^{2}=-1$ ). Assuming $\left|S_{\alpha, \beta}\right|=5$, we find for $\tau_{\alpha, \beta}$ the following eigenspaces

$$
\begin{array}{ll}
H_{3}(F)_{1}=\left\langle E_{1}, E_{2}+E_{3}\right\rangle, & H_{3}(F)_{z}=\left\langle-i^{(3)}+1^{(2)}\right\rangle, \\
H_{3}(F)_{z^{-1}}=\left\langle i^{(3)}+1^{(2)}\right\rangle, & H_{3}(F)_{z^{2}}=\left\langle-i E_{2}+i E_{3}+1^{(1)}\right\rangle, \\
H_{3}(F)_{z^{-2}}=\left\langle i E_{2}-i E_{3}+1^{(1)}\right\rangle, &
\end{array}
$$

where the subindex indicates the eigenvalue of $\tau_{\alpha, \beta}$. This gives a $\mathbb{Z}$-grading of $H_{3}(F)$, with $n$-th component $H_{3}(F)_{z^{n}}$. This is toral and fine (as it is produced by the whole torus $\mathcal{T})$. Any other toral grading of $H_{3}(F)$ is a coarsening of this.

For $\left|S_{\alpha, \beta}\right|<5$ we have the following excluding possibilities:

- $1=z$ which gives the trivial grading.

- $1=z^{2}$ which excluding the previous case implies $z=-1$. This is the $\mathbb{Z}_{2}$-grading induced by the involutive automorphism $\tau_{-1,0}$ and it is given by

$$
H_{3}(F)_{1}=\left\langle E_{1}, E_{2}, E_{3}, 1^{(1)}\right\rangle, \quad H_{3}(F)_{-1}=\left\langle 1^{(2)}, 1^{(3)}\right\rangle .
$$

- $z=z^{-2}$ implying $z^{3}=1$. Ruling out previous cases, this induces a $\mathbb{Z}_{3}$-grading coming for instance from $\tau_{-1 / 2, \sqrt{3} / 2}$. The grading is

$$
\begin{aligned}
H_{3}(F)_{1} & =\left\langle E_{1}, E_{2}+E_{3}\right\rangle, \\
H_{3}(F)_{z} & =\left\langle 1^{(2)}-i^{(3)}, i E_{2}-i E_{3}+1^{(1)}\right\rangle, \\
H_{3}(F)_{z^{2}} & =\left\langle 1^{(2)}+i^{(3)},-i E_{2}+i E_{3}+1^{(1)}\right\rangle .
\end{aligned}
$$


- $z^{2}=z^{-2}$ implying $z^{4}=1$. This gives a $\mathbb{Z}_{4}$-grading coming from $\tau_{0,1}$. The grading is

$$
\begin{array}{ll}
H_{3}(F)_{1}=\left\langle E_{1}, E_{2}+E_{3}\right\rangle, & H_{3}(F)_{z}=\left\langle-i^{(3)}+1^{(2)}\right\rangle \\
H_{3}(F)_{z^{-1}}=\left\langle i^{(3)}+1^{(2)}\right\rangle, & H_{3}(F)_{z^{2}}=\left\langle E_{2}-E_{3}, 1^{(1)}\right\rangle .
\end{array}
$$

The gradings in (3.2)-(3.5) are therefore the unique cyclic (hence necessarily toral, see Appendix) gradings. To find the rest of the gradings on $H_{3}(F)$ we compute the centralizers of the grading automorphisms producing the previous gradings. The computations of the various centralizers are easy taking advantage of the isomorphism $\mathrm{SO}(3) \rightarrow G$. For any $\tau_{\alpha, \beta}$ with $\left|S_{\alpha, \beta}\right|=5$ we have $\mathfrak{C}_{G}\left(\tau_{\alpha, \beta}\right)=\mathcal{T}$ (for this, we only need to prove that the centralizer of $p_{\alpha, \beta}$ in $\mathrm{SO}(3)$ is the maximal torus $P$ ), so that the grading (3.2) is fine, as mentioned. The centralizer of $\tau_{-1,0}$ has two connected components: the identity component is the maximal torus $\mathcal{T}$ and $\mathfrak{C}_{G}\left(\tau_{-1,0}\right) / \mathcal{T} \cong \mathbb{Z}_{2}$. Working in $\mathrm{SO}(3)$, the identity component of the centralizer of $p_{-1,0}$ is the torus $P$ while its other component is $s P$ where $s=-E_{1}+1^{(1)}$. Taking any $\tau \in \mathcal{T}$, the grading induced by $\left\{\tau_{-1,0}, \tau\right\}$ is some of (3.2)-(3.5). But if we consider the grading $\left\{\tau_{-1,0}, \operatorname{In}(s)\right\}$, we get the $\mathbb{Z}_{2} \times \mathbb{Z}_{2}$-grading given by

$$
\begin{array}{ll}
H_{1,1}=\left\langle E_{1}, E_{2}+E_{3}, 1^{(1)}\right\rangle, & H_{1,-1}=\left\langle 1^{(2)}-1^{(3)}\right\rangle, \\
H_{-1,1}=\left\langle E_{2}-E_{3}\right\rangle, & H_{-1,-1}=\left\langle 1^{(2)}+1^{(3)}\right\rangle,
\end{array}
$$

which is isomorphic to:

$$
\begin{array}{ll}
H_{1,1}=\left\langle E_{1}, E_{2}, E_{3}\right\rangle, & H_{1,-1}=\left\langle 1^{(1)}\right\rangle, \\
H_{-1,1}=\left\langle 1^{(2)}\right\rangle, & H_{-1,-1}=\left\langle 1^{(3)}\right\rangle .
\end{array}
$$

On the other hand $\mathfrak{C}_{G}\left(\left\langle\tau_{-1,0}, \operatorname{In}(s)\right\rangle\right)=\mathfrak{C}_{G}\left(\tau_{-1,0}\right)$, which implies that (3.6) is fine (and nontoral taking into account [13, Theorem 1]). The grading (3.4) is produced by $t_{-1 / 2, \sqrt{3} / 2}$ whose centralizer is $\mathcal{T}$. The grading (3.5) is produced by $\tau_{0,1}$ whose centralizer is again $\mathcal{T}$.

Summarizing all the above results we claim:

Theorem 1. Any nontrivial grading on $H_{3}(F)$ is equivalent to one of the gradings (3.2)-(3.6) above.

The gradings induced on the Albert algebra by the monomorphism from aut $\left(H_{3}(F)\right)$ to $F_{4}$ are all of them toral, but these automorphisms of $J$ coming from $H_{3}(F)$ commute with the automorphisms coming from $C$, a fact which will provide larger abelian sets of semisimple automorphisms and hence a source of nontoral gradings on $J$. 


\subsection{A family of nontoral gradings on the Albert algebra}

We now construct a machinery for building gradings on the Albert algebra $J=H_{3}(C)$ by mixing gradings on $C$ with those on $H_{3}(F)$. We must start with the simple observation that $J=H_{3}(C) \cong H_{3}(F) \otimes F \oplus K_{3}(F) \otimes C_{0}$ (tensor product of $F$-spaces) where $K_{3}(F)$ is the subspace of $3 \times 3$ skewsymmetric matrices with entries in $F$ and $C_{0}:=\{x \in C \mid \operatorname{tr}(x)=0\}$ the subspace of trace zero elements in $C$. The above isomorphism is given by $E_{i} \mapsto E_{i} \otimes 1,1^{(i)} \mapsto 1^{(i)} \otimes 1$ and for $x \in C_{0}, x^{(i)} \mapsto\left(e_{j k}-e_{k j}\right) \otimes x$, being $(i, j, k)$ any cyclic permutation of $(1,2,3)$ and $e_{i j}$ the elementary $(i, j)$-matrix in $M_{3}(F)$. Taking in $J^{\prime}=H_{3}(F) \otimes F \oplus K_{3}(F) \otimes C_{0}$, which is a subspace of $M_{3}(F) \otimes C$, the product $(c \otimes x) \cdot(d \otimes y)=\frac{1}{2}((c \otimes x)(d \otimes y)+(d \otimes y)(c \otimes x))$ for $(c \otimes x)(d \otimes y)=c d \otimes x y, J^{\prime}$ is a Jordan subalgebra of $M_{3}(F) \otimes C$ such that the previous vector space isomorphism between $J$ and $J^{\prime}$ is an algebra isomorphism. Module this identification of $J$ with $J^{\prime}$, the embedding of $G_{2}$ in $F_{4}$ described in Subsection 3.1 can be seen in the following way. Given $f \in G_{2}$, take $\hat{f}$ the restriction of id $\otimes f \in \operatorname{GL}\left(M_{3}(F) \otimes C\right)$ to $J^{\prime}$, which is an automorphism of $J^{\prime}$ (notice that $C_{0}$ is $f$-invariant for any $f \in G_{2}$ ). On the other hand given any automorphism $g$ of $H_{3}(F), g$ is the restriction to $H_{3}(F)$ of an automorphism $g$ of $M_{3}(F)$ commuting with the transposition involution. Hence $g\left(K_{3}(F)\right) \subset K_{3}(F)$ and we can define $\tilde{g}$ as the restriction of $g \otimes$ id $\in \mathrm{GL}\left(M_{3}(F) \otimes C\right)$ to $J^{\prime}$, which is an automorphism of $J^{\prime}$. A trivial though remarkable fact is the commutativity $\hat{f} \tilde{g}=\tilde{g} \hat{f}$ for any $f \in G_{2}$, $g \in \operatorname{aut}\left(H_{3}(F)\right)$. Thus we have:

Theorem 2. Let $\left\{f_{1}, \ldots, f_{k}\right\} \subset G_{2}$ and $\left\{g_{1}, \ldots, g_{n}\right\} \subset \operatorname{aut}\left(H_{3}(F)\right)$ be commuting sets of diagonalizable automorphisms. Then $\left\{\widehat{f}_{1}, \ldots, \widehat{f}_{k}, \widetilde{g}_{1}, \ldots, \widetilde{g}_{n}\right\} \subset$ $F_{4}$ is a commuting set of diagonalizable automorphisms of $J$. In particular if $C$ is graded by a group $G_{1}$ and $H_{3}(F)$ is graded by a second group $G_{2}$, then the Albert algebra $J$ is $G_{1} \times G_{2}$-graded.

It is always the case that the grading induced by $\left\{\widehat{f}_{1}, \ldots, \widehat{f_{k}}, \widetilde{g_{1}}, \ldots, \widetilde{g_{n}}\right\}$ is a refinement of the one given by $\left\{\widehat{f}_{1}, \ldots, \widehat{f}_{k}\right\}$. Besides if one of the gradings $\left\{\widehat{f}_{1}, \ldots, \widehat{f}_{k}\right\}$ or $\left\{\widetilde{g}_{1}, \ldots, \widetilde{g}_{n}\right\}$ is nontoral, the refinement is also nontoral. These results allow us to combine gradings on $C$ with gradings on $H_{3}(F)$. Thus, if we pick the (unique up to equivalence) nontoral grading on $C$ given by $\left\{t_{1,-1}, t_{-1,1}, f_{0}\right\}$ and any of the gradings (3.2)-(3.6) plus the trivial grading, which are given respectively by: $\left\{\tau_{\alpha, \beta}\right\}$ (with $\left|S_{\alpha \beta}\right|=5$ ), $\left\{\tau_{-1,0}\right\}$, $\left\{\tau_{-1 / 2, \sqrt{3} / 2}\right\},\left\{\tau_{0,1}\right\},\left\{\tau_{-1,0}, \operatorname{In}(s)\right\}$ and $\{1\}$, we get six nontoral gradings on $J$ which are given in the next result. 
Corollary 1. The following six nonequivalent gradings on the Albert algebra are nontoral:

a) $\left\{\widehat{t_{1,-1}}, \widehat{t_{-1,1}}, \widehat{f_{0}}, \widetilde{\tau_{\alpha, \beta}}\right\}$ with $\left|S_{\alpha, \beta}\right|=5$. This is a $\mathbb{Z}_{2}^{3} \times \mathbb{Z}$-grading.

b) $\left\{\widehat{t_{1,-1}}, \widehat{t_{-1,1}}, \widehat{f_{0}}, \widetilde{\tau_{-1,0}}\right\}$. This is a $\mathbb{Z}_{2}^{4}$-grading.

c) $\left\{\widehat{t_{1,-1}}, \widehat{t_{-1,1}}, \widehat{f_{0}}, \widehat{\tau_{-\frac{1}{2}, \frac{\sqrt{3}}{2}}}\right\}$. This is a $\mathbb{Z}_{2}^{3} \times \mathbb{Z}_{3}$-grading.

d) $\left\{\widehat{t_{1,-1}}, \widehat{t_{-1,1}}, \widehat{f_{0}}, \widetilde{\tau_{0,1}}\right\}$. This is a $\mathbb{Z}_{2}^{3} \times \mathbb{Z}_{4}$-grading .

e) $\left\{\widehat{t_{1,-1}}, \widehat{t_{-1,1}}, \widehat{f_{0}}, \widetilde{\tau_{-1,0}}, \widehat{\operatorname{In}(s)}\right\}$. This is a $\mathbb{Z}_{2}^{5}$-grading.

f) $\left\{\widehat{t_{1,-1}}, \widehat{t_{-1,1}}, \widehat{f_{0}}\right\}$. This is a $\mathbb{Z}_{2}^{3}$-grading.

We now describe explicitly the six previous gradings.

a) If $\epsilon= \pm 1$ then the grading is

$$
\begin{array}{rlrl}
J_{0000}=\left\langle E_{1}, E_{2}+E_{3}\right\rangle, & J_{000 \epsilon}=\left\langle-i \epsilon^{(3)}+1^{(2)}\right\rangle, \\
J_{001 \epsilon}=\left\langle i \epsilon\left(e_{1}-e_{2}\right)^{(3)}+\left(e_{1}-e_{2}\right)^{(2)}\right\rangle, & & J_{0010}=\left\langle\left(-e_{1}+e_{2}\right)^{(1)}\right\rangle, \\
J_{010 \epsilon}=\left\langle-i \epsilon\left(u_{2}+v_{2}\right)^{(3)}-\left(u_{2}+v_{2}\right)^{(2)}\right\rangle, & & J_{0100}=\left\langle\left(u_{2}+v_{2}\right)^{(1)}\right\rangle, \\
J_{100 \epsilon}=\left\langle-i \epsilon\left(u_{1}+v_{1}\right)^{(3)}-\left(u_{1}+v_{1}\right)^{(2)}\right\rangle, & & J_{1000}=\left\langle\left(u_{1}+v_{1}\right)^{(1)}\right\rangle, \\
J_{011 \epsilon}=\left\langle i \epsilon\left(u_{2}-v_{2}\right)^{(3)}+\left(u_{2}-v_{2}\right)^{(2)}\right\rangle, & J_{0110}=\left\langle\left(u_{2}-v_{2}\right)^{(1)}\right\rangle, \\
J_{110 \epsilon}=\left\langle i \epsilon\left(u_{3}-v_{3}\right)^{(3)}+\left(u_{3}-v_{3}\right)^{(2)}\right\rangle, & J_{1100}=\left\langle\left(u_{3}-v_{3}\right)^{(1)}\right\rangle, \\
J_{101 \epsilon}=\left\langle i \epsilon\left(u_{1}-v_{1}\right)^{(3)}+\left(u_{1}-v_{1}\right)^{(2)}\right\rangle, & J_{1010}=\left\langle\left(u_{1}-v_{1}\right)^{(1)}\right\rangle, \\
J_{111 \epsilon}=\left\langle-i \epsilon\left(u_{3}+v_{3}\right)^{(3)}-\left(u_{3}+v_{3}\right)^{(2)}\right\rangle, & J_{1110}=\left\langle\left(u_{3}+v_{3}\right)^{(1)}\right\rangle, \\
J_{0002}=\left\langle-i\left(E_{2}-E_{3}\right)+1^{(1)}\right\rangle, & J_{000-2}=\left\langle-i\left(E_{2}-E_{3}\right)-1^{(1)}\right\rangle .
\end{array}
$$

This grading is a $\mathbb{Z}_{2}^{3} \times \mathbb{Z}$-grading of type $(25,1)$.

b) This is the $\mathbb{Z}_{2}^{4}$-grading

$$
\begin{array}{rlrl}
J_{0000}=\left\langle E_{1}, E_{2}, E_{3}, 1^{(1)}\right\rangle, & J_{0001} & =\left\langle 1^{(3)}, 1^{(2)}\right\rangle, \\
J_{0010}=\left\langle\left(e_{1}-e_{2}\right)^{(1)}\right\rangle, & J_{0100}=\left\langle\left(u_{2}+v_{2}\right)^{(1)}\right\rangle, \\
J_{1000}=\left\langle\left(u_{1}+v_{1}\right)^{(1)}\right\rangle, & J_{1100}=\left\langle\left(u_{3}-v_{3}\right)^{(1)}\right\rangle, \\
J_{1010}=\left\langle\left(u_{1}-v_{1}\right)^{(1)}\right\rangle, & J_{1001}=\left\langle\left(u_{1}+v_{1}\right)^{(3)},\left(u_{1}+v_{1}\right)^{(2)}\right\rangle, \\
J_{0110}=\left\langle\left(-u_{2}+v_{2}\right)^{(1)}\right\rangle, & J_{0101}=\left\langle\left(u_{2}+v_{2}\right)^{(3)},\left(u_{2}+v_{2}\right)^{(2)}\right\rangle, \\
J_{0011}=\left\langle\left(e_{1}-e_{2}\right)^{(3)},\left(e_{1}-e_{2}\right)^{(2)}\right\rangle, & J_{1110}=\left\langle\left(u_{3}+v_{3}\right)^{(1)}\right\rangle, \\
J_{1101}=\left\langle\left(u_{3}-v_{3}\right)^{(3)},\left(u_{3}-v_{3}\right)^{(2)}\right\rangle, & J_{1011}=\left\langle\left(u_{1}-v_{1}\right)^{(3)},\left(u_{1}-v_{1}\right)^{(2)}\right\rangle, \\
J_{0111}=\left\langle\left(u_{2}-v_{2}\right)^{(3)},\left(-u_{2}+v_{2}\right)^{(2)}\right\rangle, & J_{1111}=\left\langle\left(u_{3}+v_{3}\right)^{(3)},\left(u_{3}+v_{3}\right)^{(2)}\right\rangle,
\end{array}
$$

which is of type $(7,8,0,1)$. 
c) This is the $\mathbb{Z}_{2}^{3} \times \mathbb{Z}_{3}$-grading given by

$$
\begin{array}{rlrl}
J_{0000} & =\left\langle E_{1}, E_{2}+E_{3}\right\rangle, & & J_{1000}=\left\langle\left(u_{1}+v_{1}\right)^{(1)}\right\rangle, \\
J_{0100} & =\left\langle\left(u_{2}+v_{2}\right)^{(1)}\right\rangle, & & J_{0010}=\left\langle\left(e_{1}-e_{2}\right)^{(1)}\right\rangle, \\
J_{0110} & =\left\langle\left(u_{2}-v_{2}\right)^{(1)}\right\rangle, & & J_{1010}=\left\langle\left(u_{1}-v_{1}\right)^{(1)}\right\rangle, \\
J_{1100}=\left\langle\left(u_{3}-v_{3}\right)^{(1)}\right\rangle, & & J_{1110}=\left\langle\left(u_{3}+v_{3}\right)^{(1)}\right\rangle,
\end{array}
$$

$J_{0001}=\left\langle-i 1^{(3)}+1^{(2)}, i E_{2}-i E_{3}+1^{(1)}\right\rangle, \quad J_{1001}=\left\langle i\left(u_{1}+v_{1}\right)^{(3)}+\left(u_{1}+v_{1}\right)^{(2)}\right\rangle$,

$$
\begin{array}{ll}
J_{0101}=\left\langle i\left(u_{2}+v_{2}\right)^{(3)}+\left(u_{2}+v_{2}\right)^{(2)}\right\rangle, & J_{0011}=\left\langle i\left(e_{1}-e_{2}\right)^{(3)}+\left(e_{1}-e_{2}\right)^{(2)}\right\rangle, \\
J_{0111}=\left\langle i\left(u_{2}-v_{2}\right)^{(3)}+\left(u_{2}-v_{2}\right)^{(2)}\right\rangle, & J_{1011}=\left\langle i\left(u_{1}-v_{1}\right)^{(3)}+\left(u_{1}-v_{1}\right)^{(2)}\right\rangle,
\end{array}
$$$$
J_{1101}=\left\langle i\left(u_{3}-v_{3}\right)^{(3)}+\left(u_{3}-v_{3}\right)^{(2)}\right\rangle, \quad J_{1111}=\left\langle i\left(u_{3}+v_{3}\right)^{(3)}+\left(u_{3}+v_{3}\right)^{(2)}\right\rangle \text {, }
$$$$
J_{0002}=\left\langle i 1^{(3)}+1^{(2)}, i E_{2}-i E_{3}-1^{(1)}\right\rangle, \quad J_{1002}=\left\langle i\left(u_{1}+v_{1}\right)^{(3)}-\left(u_{1}+v_{1}\right)^{(2)}\right\rangle,
$$$$
J_{0102}=\left\langle i\left(u_{2}+v_{2}\right)^{(3)}-\left(u_{2}+v_{2}\right)^{(2)}\right\rangle, \quad J_{0012}=\left\langle-i\left(e_{1}-e_{2}\right)^{(3)}+\left(e_{1}-e_{2}\right)^{(2)}\right\rangle,
$$$$
J_{0112}=\left\langle i\left(u_{2}-v_{2}\right)^{(3)}-\left(u_{2}-v_{2}\right)^{(2)}\right\rangle, \quad J_{1012}=\left\langle-i\left(u_{1}-v_{1}\right)^{(3)}+\left(u_{1}-v_{1}\right)^{(2)}\right\rangle \text {, }
$$$$
J_{1102}=\left\langle i\left(u_{3}-v_{3}\right)^{(3)}-\left(u_{3}-v_{3}\right)^{(2)}\right\rangle, \quad J_{1112}=\left\langle i\left(u_{3}+v_{3}\right)^{(3)}-\left(u_{3}+v_{3}\right)^{(2)}\right\rangle \text {, }
$$

which is of type $(21,3)$.

d) This is the $\mathbb{Z}_{2}^{3} \times \mathbb{Z}_{4}$-grading

$$
\begin{aligned}
J_{0000}=\left\langle E_{1}, E_{2}+E_{3}\right\rangle, & J_{1000}=\left\langle\left(u_{1}+v_{1}\right)^{(1)}\right\rangle, \\
J_{0100}=\left\langle\left(u_{2}+v_{2}\right)^{(1)}\right\rangle, & J_{0010}=\left\langle\left(e_{1}-e_{2}\right)^{(1)}\right\rangle, \\
J_{0110}=\left\langle\left(u_{2}-v_{2}\right)^{(1)}\right\rangle, & J_{1010}=\left\langle\left(u_{1}-v_{1}\right)^{(1)}\right\rangle, \\
J_{1100}=\left\langle\left(u_{3}-v_{3}\right)^{(1)}\right\rangle, & J_{1110}=\left\langle\left(u_{3}+v_{3}\right)^{(1)}\right\rangle, \\
J_{0001}=\left\langle-i 1^{(3)}+1^{(2)}\right\rangle, & J_{1001}=\left\langle i\left(u_{1}+v_{1}\right)^{(3)}+\left(u_{1}+v_{1}\right)^{(2)}\right\rangle, \\
J_{0101}=\left\langle i\left(u_{2}+v_{2}\right)^{(3)}+\left(u_{2}+v_{2}\right)^{(2)}\right\rangle, & J_{0011}=\left\langle i\left(e_{1}-e_{2}\right)^{(3)}+\left(e_{1}-e_{2}\right)^{(2)}\right\rangle, \\
J_{0111}=\left\langle i\left(u_{2}-v_{2}\right)^{(3)}+\left(u_{2}-v_{2}\right)^{(2)}\right\rangle, & J_{1011}=\left\langle i\left(u_{1}-v_{1}\right)^{(3)}+\left(u_{1}-v_{1}\right)^{(2)}\right\rangle, \\
J_{1101}=\left\langle i\left(u_{3}-v_{3}\right)^{(3)}+\left(u_{3}-v_{3}\right)^{(2)}\right\rangle, & J_{1111}=\left\langle i\left(u_{3}+v_{3}\right)^{(3)}+\left(u_{3}+v_{3}\right)^{(2)}\right\rangle, \\
J_{0002}=\left\langle E_{3}-E_{2}, 1^{(1)}\right\rangle, & J_{0003}=\left\langle i 1^{(3)}+1^{(2)}\right\rangle, \\
J_{1003}=\left\langle i\left(u_{1}+v_{1}\right)^{(3)}-\left(u_{1}+v_{1}\right)^{(2)}\right\rangle, & J_{0103}=\left\langle i\left(u_{2}+v_{2}\right)^{(3)}-\left(u_{2}+v_{2}\right)^{(2)}\right\rangle, \\
J_{0013}=\left\langle-i\left(e_{1}-e_{2}\right)^{(3)}+\left(e_{1}-e_{2}\right)^{(2)}\right\rangle, & J_{0113}=\left\langle-i\left(u_{2}-v_{2}\right)^{(3)}+\left(u_{2}-v_{2}\right)^{(2)}\right\rangle, \\
J_{1013}=\left\langle-i\left(u_{1}-v_{1}\right)^{(3)}+\left(u_{1}-v_{1}\right)^{(2)}\right\rangle, & J_{1103}=\left\langle-i\left(u_{3}-v_{3}\right)^{(3)}+\left(u_{3}-v_{3}\right)^{(2)}\right\rangle, \\
& J_{1113}=\left\langle i\left(u_{3}+v_{3}\right)^{(3)}-\left(u_{3}+v_{3}\right)^{(2)}\right\rangle,
\end{aligned}
$$

which is of type $(23,2)$.

e) This is the $\mathbb{Z}_{2}^{5}$-grading given by

$$
\begin{aligned}
J_{00000}=\left\langle E_{1}, E_{2}+E_{3}, 1^{(1)}\right\rangle, & J_{00010} & =\left\langle-1^{(3)}+1^{(2)}\right\rangle, \\
J_{00001}=\left\langle E_{3}-E_{2}\right\rangle, & & J_{10010}=\left\langle\left(u_{1}+v_{1}\right)^{(3)}+\left(u_{1}+v_{1}\right)^{(2)}\right\rangle, \\
J_{10001}=\left\langle\left(u_{1}+v_{1}\right)^{(1)}\right\rangle, & & J_{01010}=\left\langle\left(u_{2}+v_{2}\right)^{(3)}+\left(u_{2}+v_{2}\right)^{(2)}\right\rangle, \\
J_{01001}=\left\langle\left(u_{2}+v_{2}\right)^{(1)}\right\rangle, & & J_{00110}=\left\langle\left(e_{1}-e_{2}\right)^{(3)}+\left(e_{1}-e_{2}\right)^{(2)}\right\rangle,
\end{aligned}
$$




$$
\begin{aligned}
J_{00101}=\left\langle\left(-e_{1}+e_{2}\right)^{(1)}\right\rangle, & J_{00011}=\left\langle 1^{(3)}+1^{(2)}\right\rangle, \\
J_{00111}=\left\langle\left(e_{2}-e_{1}\right)^{(3)}+\left(e_{1}-e_{2}\right)^{(2)}\right\rangle, & J_{01011}=\left\langle\left(u_{2}+v_{2}\right)^{(3)}-\left(u_{2}+v_{2}\right)^{(2)}\right\rangle, \\
J_{01101}=\left\langle\left(u_{2}-v_{2}\right)^{(1)}\right\rangle, & J_{01110}=\left\langle\left(u_{2}-v_{2}\right)^{(3)}+\left(u_{2}-v_{2}\right)^{(2)}\right\rangle, \\
J_{01111}=\left\langle\left(-u_{2}+v_{2}\right)^{(3)}+\left(u_{2}-v_{2}\right)^{(2)}\right\rangle, & J_{10011}=\left\langle\left(u_{1}+v_{1}\right)^{(3)}-\left(u_{1}+v_{1}\right)^{(2)}\right\rangle, \\
J_{10101}=\left\langle\left(u_{1}-v_{1}\right)^{(1)}\right\rangle, & J_{10110}=\left\langle\left(u_{1}-v_{1}\right)^{(3)}+\left(u_{1}-v_{1}\right)^{(2)}\right\rangle, \\
J_{10111}=\left\langle\left(-u_{1}+v_{1}\right)^{(3)}+\left(u_{1}-v_{1}\right)^{(2)}\right\rangle, & J_{11001}=\left\langle\left(u_{3}-v_{3}\right)^{(1)}\right\rangle, \\
J_{11010}=\left\langle\left(u_{3}-v_{3}\right)^{(3)}+\left(u_{3}-v_{3}\right)^{(2)}\right\rangle, & J_{11011}=\left\langle\left(-u_{3}+v_{3}\right)^{(3)}+\left(u_{3}-v_{3}\right)^{(2)}\right\rangle, \\
J_{11101}=\left\langle\left(u_{3}+v_{3}\right)^{(1)}\right\rangle, & J_{11110}=\left\langle\left(-u_{3}-v_{3}\right)^{(3)}-\left(u_{3}+v_{3}\right)^{(2)}\right\rangle, \\
& J_{11111}=\left\langle\left(u_{3}+v_{3}\right)^{(3)}-\left(u_{3}+v_{3}\right)^{(2)}\right\rangle,
\end{aligned}
$$

which is of type $(24,0,1)$.

f) This is the $\mathbb{Z}_{2}^{3}$-grading (3.1), which is nontoral of type $(0,0,7,0,0,1)$, as mentioned in Proposition 2.

Before finishing this subsection we would like to exhibit another (nontoral) grading obtained as a coarsening of the one in case d) above. Thus consider the grading on the Albert algebra induced by the automorphisms $\left\{\widehat{t_{1,-1}}, \widehat{t_{-1,1}}, \widehat{f_{0}} \widetilde{\tau_{0,1}}\right\}$. This is the $\mathbb{Z}_{2}^{2} \times \mathbb{Z}_{4}$-grading whose homogeneous components are:

$$
\begin{aligned}
J_{000}=\left\langle E_{1}, E_{2}+E_{3}\right\rangle, & J_{001}=\left\langle i e_{1}^{(3)}-e_{1}^{(2)}, i e_{2}^{(3)}-e_{2}^{(2)}\right\rangle, \\
J_{002}=\left\langle e_{1}^{(1)}, E_{3}-E_{2}, e_{2}^{(1)}\right\rangle, & J_{003}=\left\langle i e_{1}^{(3)}+e_{1}^{(2)}, i e_{2}^{(3)}+e_{2}^{(2)}\right\rangle, \\
J_{010}=\left\langle\left(u_{2}+v_{2}\right)^{(1)}\right\rangle, & J_{012}=\left\langle\left(u_{2}-v_{2}\right)^{(1)}\right\rangle, \\
J_{011}=\left\langle i v_{2}^{(3)}+u_{2}^{(2)}, i u_{2}^{(3)}+v_{2}^{(2)}\right\rangle, & J_{013}=\left\langle i u_{2}^{(3)}-v_{2}^{(2)}, i v_{2}^{(3)}-u_{2}^{(2)}\right\rangle, \\
J_{100}=\left\langle\left(u_{1}+v_{1}\right)^{(1)}\right\rangle, & J_{102}=\left\langle\left(u_{1}-v_{1}\right)^{(1)}\right\rangle, \\
J_{101}=\left\langle i v_{1}^{(3)}+u_{1}^{(2)}, i u_{1}^{(3)}+v_{1}^{(2)}\right\rangle, & J_{103}=\left\langle i u_{1}^{(3)}-v_{1}^{(2)}, i v_{1}^{(3)}-u_{1}^{(2)}\right\rangle, \\
J_{110}=\left\langle\left(u_{3}-v_{3}\right)^{(1)}\right\rangle, & J_{112}=\left\langle\left(u_{3}+v_{3}\right)^{(1)}\right\rangle, \\
J_{111}=\left\langle i u_{3}^{(3)}-v_{3}^{(2)}, i v_{3}^{(3)}-u_{3}^{(2)}\right\rangle, & J_{113}=\left\langle i v_{3}^{(3)}+u_{3}^{(2)}, i u_{3}^{(3)}+v_{3}^{(2)}\right\rangle,
\end{aligned}
$$

which is of type $(6,9,1)$. Note that it is nontoral too because toral gradings have at least three division orthogonal idempotents in its zero component.

\subsection{Gradings from $M_{3}(F)$}

Up to the moment we have detected seven equivalence classes of nontoral gradings on $J$, all of them coming from the refinements of the nontoral grading on $C$ by gradings on $H_{3}(F)$. In order to find new nontoral gradings on the Albert algebra we need to look at $J$ from another point of view, that is, we can use a different model of $J$ which provides a new perspective. For instance, Tits construction of $J$ recalled in Subsection 2.4. 
Thus let us consider again the associative algebra $A:=M_{3}(F)$ and the monomorphism $\iota$ : $\operatorname{aut}(A) \rightarrow F_{4}$ such that $f \mapsto f^{\bullet}$ as described in 2.4. If $\left\{f_{i}\right\}$ is a finite commutative family of semisimple automorphisms of $A$, the same is true for the family $\left\{f_{i}^{\bullet}\right\}$. Hence, for a $G$-grading on $A$ given by a group homomorphism $\rho: \mathfrak{X}(G) \rightarrow$ aut $(A)$ we immediately can define the grading on $J$ given by $\iota \rho: \mathfrak{X}(G) \rightarrow F_{4}$. Consider now the $\mathbb{Z}_{3}^{2}$-grading on $A$ produced by the commuting automorphisms $f:=\operatorname{In}(p)$ and $g:=\operatorname{In}(q)$ where $p=\operatorname{diag}\left(1, \omega, \omega^{2}\right)$ being $\omega$ a primitive cubic root of the unit and

$$
q=\left(\begin{array}{lll}
0 & 1 & 0 \\
0 & 0 & 1 \\
1 & 0 & 0
\end{array}\right)
$$

These automorphisms of $A$ are semisimple of order 3 . The group they generate, $\langle f, g\rangle$, is usually called Pauli group. The simultaneous diagonalization of $A$ relative to $\{f, g\}$ yields $A=\oplus_{i, j=0}^{2} A_{i j}$ for

$$
\begin{array}{rll}
A_{00}=\left\langle 1_{A}\right\rangle, & A_{01}=\left\langle\omega^{2} e_{11}-\omega e_{22}+e_{33}\right\rangle, & A_{02}=\left\langle-\omega e_{11}+\omega^{2} e_{22}+e_{33}\right\rangle, \\
A_{10}=\left\langle e_{13}+e_{21}+e_{32}\right\rangle, & A_{11}=\left\langle\omega^{2} e_{13}-\omega e_{21}+e_{32}\right\rangle, & A_{12}=\left\langle-\omega e_{13}+\omega^{2} e_{21}+e_{32}\right\rangle, \\
A_{20}=\left\langle e_{12}+e_{23}+e_{31}\right\rangle, & A_{21}=\left\langle\omega^{2} e_{12}-\omega e_{23}+e_{31}\right\rangle, & A_{22}=\left\langle-\omega e_{12}+\omega^{2} e_{23}+e_{31}\right\rangle,
\end{array}
$$

where again $e_{i j}$ denotes the elementary $(i, j)$-matrix in $M_{3}(F)$. Thus we have a $\mathbb{Z}_{3}^{2}$-nontoral grading on $A$ (since any maximal torus of $A$ fixes a frame of idempotents and so any toral grading has a zero component of dimension at least 3). Next we can consider the grading induced on $J$ by $\left\{f^{\bullet}, g^{\bullet}\right\}$. If we make a simultaneous diagonalization of $J$ relative to these automorphisms we get the $\mathbb{Z}_{3}^{2}$-grading $J=\oplus_{i, j=0}^{2} A_{i, j}^{3}$, which has 9 summands of dimension 3 each one. This $\mathbb{Z}_{3}^{2}$-grading on $J$ is obviously toral according to Lemma 2 in the Appendix. Let us consider a third semisimple automorphism $\phi$ of order 3 in the centralizer of $\left\{f^{\bullet}, g^{\bullet}\right\}$. This will allow us to refine the previous $\mathbb{Z}_{3}^{2}$-grading on $J$ to a $\mathbb{Z}_{3}^{3}$-grading. So consider $\phi \in \operatorname{aut}(J)$ given by $\phi\left(a_{0}, a_{1}, a_{2}\right)=\left(a_{0}, \omega a_{1}, \omega^{2} a_{2}\right)$ where $\omega$ is as before a primitive cubic root of the unit. It is clear that $\left\{f^{\bullet}, g^{\bullet}, \phi\right\}$ is a commutative set of semisimple automorphisms of $J$. Making again a simultaneous diagonalization of $J$ relative to $\left\{f^{\bullet}, g^{\bullet}, \phi\right\}$ we get $J=\bigoplus_{i, j, k=0}^{2} J_{i, j, k}$ with

$$
\begin{aligned}
& J_{i, j, 0}=A_{i j} \times 0 \times 0, \\
& J_{i, j, 1}=0 \times A_{i j} \times 0, \\
& J_{i, j, 2}=0 \times 0 \times A_{i j},
\end{aligned}
$$

so that we have 27 one-dimensional homogeneous components. In particular this $\mathbb{Z}_{3}^{3}$-grading on $J$ is fine and nontoral (otherwise $J_{0,0,0}$ would contain three orthogonal idempotents, by Proposition 1). Consequently, the subgroup $\left\langle f^{\bullet}, g^{\bullet}, \phi\right\rangle$ of $F_{4}$ is maximal among the abelian subgroups of $F_{4}$ whose 
elements are semisimple (MAD). Observe also that the generators of the subspaces $A_{i j}$ of $A$ are invertible elements in $A$, hence taking into account (2.4), the generators of the homogeneous components $J_{i, j, k}$ are also invertible in $J$. Thus we have found a basis of invertible homogeneous elements in the Albert algebra.

Since we are describing gradings on the Albert algebra in the usual standard basis and this last $\mathbb{Z}_{3}^{3}$-grading has been given in a different one, we are now giving the mentioned grading relative to some standard basis. We take, for instance, the grading:

$$
\begin{array}{rlrl}
J_{000}=\left\langle E_{1}+E_{2}+E_{3}\right\rangle, & J_{001}=\left\langle\omega E_{1}+\omega^{2} E_{2}+E_{3}\right\rangle, \\
J_{002}=\left\langle\omega^{2} E_{1}+\omega E_{2}+E_{3}\right\rangle, & J_{010}=\left\langle u_{3}^{(3)}+e_{1}^{(2)}+v_{3}^{(1)}\right\rangle, \\
J_{011}=\left\langle\omega^{2} u_{3}^{(3)}+\omega e_{1}^{(2)}+v_{3}^{(1)}\right\rangle, & J_{012}=\left\langle\omega u_{3}^{(3)}+\omega^{2} e_{1}^{(2)}+v_{3}^{(1)}\right\rangle, \\
J_{020}=\left\langle v_{3}^{(3)}-e_{2}^{(2)}+u_{3}^{(1)}\right\rangle, & J_{021}=\left\langle\omega^{2} v_{3}^{(3)}-\omega e_{2}^{(2)}+u_{3}^{(1)}\right\rangle, \\
J_{022}=\left\langle\omega v_{3}^{(3)}-\omega^{2} e_{2}^{(2)}+u_{3}^{(1)}\right\rangle, & J_{100}=\left\langle-v_{2}^{(3)}-u_{2}^{(2)}+e_{1}^{(1)}\right\rangle, \\
J_{101}=\left\langle-\omega^{2} v_{2}^{(3)}-\omega u_{2}^{(2)}+e_{1}^{(1)}\right\rangle, & J_{102}=\left\langle-\omega v_{2}^{(3)}-\omega^{2} u_{2}^{(2)}+e_{1}^{(1)}\right\rangle, \\
J_{110}=\left\langle e_{2}^{(3)}-u_{1}^{(2)}+v_{1}^{(2)}\right\rangle, & J_{111}=\left\langle\omega^{2} e_{2}^{(3)}-\omega u_{1}^{(2)}+v_{1}^{(1)}\right\rangle, \\
J_{112}=\left\langle\omega e_{2}^{(3)}-\omega^{2} u_{1}^{(2)}+v_{1}^{(1)}\right\rangle, & J_{120}=\left\langle v_{1}^{(3)}+v_{3}^{(2)}+v_{2}^{(1)}\right\rangle, \\
J_{121}=\left\langle\omega^{2} v_{1}^{(3)}+\omega v_{3}^{(2)}+v_{2}^{(1)}\right\rangle, & J_{122}=\left\langle\omega v_{1}^{(3)}+\omega^{2} v_{3}^{(2)}+v_{2}^{(1)}\right\rangle, \\
J_{200}=\left\langle u_{2}^{(3)}+v_{2}^{(2)}+e_{2}^{(1)}\right\rangle, & J_{201}=\left\langle\omega^{2} u_{2}^{(3)}+\omega v_{2}^{(2)}+e_{2}^{(1)}\right\rangle, \\
J_{202}=\left\langle\omega u_{2}^{(3)}+\omega^{2} v_{2}^{(2)}+e_{2}^{(1)}\right\rangle, & J_{210}=\left\langle u_{1}^{(3)}+u_{3}^{(2)}+u_{2}^{(1)}\right\rangle, \\
J_{211}=\left\langle\omega^{2} u_{1}^{(3)}+\omega u_{3}^{(2)}+u_{2}^{(1)}\right\rangle, & J_{212}=\left\langle\omega u_{1}^{(3)}+\omega^{2} u_{3}^{(2)}+u_{2}^{(1)}\right\rangle, \\
J_{220}=\left\langle-e_{1}^{(3)}-v_{1}^{(2)}+u_{1}^{(1)}\right\rangle, & J_{221}=\left\langle-\omega^{2} e_{1}^{(3)}-\omega v_{1}^{(2)}+u_{1}^{(1)}\right\rangle, \\
J_{222}=\left\langle-\omega e_{1}^{(3)}-\omega^{2} v_{1}^{(2)}+u_{1}^{(1)}\right\rangle . & &
\end{array}
$$

It is produced by the set of commuting diagonalizable automorphisms

$$
\left\{t_{\omega^{2}, \omega^{2}, \omega^{2}, 1}, t_{\omega^{2}, \omega, 1, \omega^{2}}, \varphi\right\},
$$

where $\varphi=\theta \circ \Psi_{U}$ for $U \in \mathrm{O}(C, n)$ is given by the matrix relative to the standard basis of $C$

$$
\left(\begin{array}{cccccccc}
0 & 0 & -1 & 0 & 0 & 0 & 0 & 0 \\
0 & 0 & 0 & 0 & 0 & 1 & 0 & 0 \\
0 & 0 & 0 & 1 & 0 & 0 & 0 & 0 \\
0 & 1 & 0 & 0 & 0 & 0 & 0 & 0 \\
0 & 0 & 0 & 0 & 0 & 0 & 0 & 1 \\
0 & 0 & 0 & 0 & 0 & 0 & 1 & 0 \\
-1 & 0 & 0 & 0 & 0 & 0 & 0 & 0 \\
0 & 0 & 0 & 0 & 1 & 0 & 0 & 0
\end{array}\right)
$$

following the notations in 2.3. This $\mathbb{Z}_{3}^{3}$-grading is fine and nontoral, since all the homogeneous components are one-dimensional. Following [19, (7.4) 
THEOREM, p. 278] for odd prime $p$ there is a unique conjugacy class of elementary abelian nontoral $p$-subgroup. This is obtained for $p=3$ and it is isomorphic to $\mathbb{Z}_{3}^{3}$ (see also [19, TABLE II, p.258]). Therefore the previous $\mathbb{Z}_{3}^{3}$-gradings on the Albert algebra are isomorphic.

In Section 6 we shall be able to give another description of this grading in terms of the Weyl group of $F_{4}$. Besides, the uniqueness of the $\mathbb{Z}_{3}^{3}$-grading will also be a consequence.

Once we have described the previous gradings on the Albert algebra, we can announce the first of our main results:

Theorem 3. Up to equivalence, the only nontoral gradings on the Albert algebra are those described in (3.7), (3.8), (3.9), (3.10), (3.11), (3.1), (3.12) and (3.13).

The nontoral fine gradings on $J$ are the ones described in (3.7), (3.11) and (3.13).

In fact, there are four fine gradings, taking into account that the Cartan decomposition is a toral and fine grading on $\mathfrak{f}_{4}$ which induces a toral and fine grading on $J$. The proof of the above theorem will have to be postponed to a forthcoming section.

\section{Weyl group of $\mathfrak{f}_{4}$}

In the next sections we shall use the Weyl group as an important tool for our purposes. First of all we must invoke a version of the Borel-Serre theorem (Theorem 6) asserting that a supersolvable subgroup of semisimple elements in an algebraic group is contained in the normalizer of some maximal torus. In particular, this can be applied to finitely generated abelian groups. The point of this is that most of our arguments can be carried out within the normalizer of a maximal torus, hence the relevance of the Weyl group, which in our context is isomorphic to the quotient of the normalizer of any maximal torus by the torus itself.

In order to describe the abstract Weyl group of $\mathfrak{f}_{4}$, we must begin by fixing a basis $\Delta=\left\{\alpha_{i} \mid i=1, \ldots, 4\right\}$ of a root system of $\mathfrak{f}_{4}$. Its Dynkin diagram is

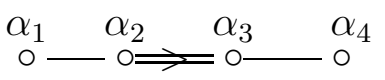

and its Cartan matrix is

$$
\left(\begin{array}{cccc}
2 & -1 & 0 & 0 \\
-1 & 2 & -2 & 0 \\
0 & -1 & 2 & -1 \\
0 & 0 & -1 & 2
\end{array}\right)
$$


Take the euclidean space $E=\sum_{i=1}^{4} \mathbb{R} \alpha_{i}$ with the inner product $(, \quad)$ described for instance in [25, Section 8]. The Weyl group of $\mathfrak{f}_{4}$ is the subgroup $\mathcal{W}$ of $\mathrm{GL}(E)$ generated by the (simple) reflections $s_{i}$ with $i=1,2,3,4$, given by

$$
s_{i}(x):=x-\left\langle x, \alpha_{i}\right\rangle \alpha_{i}, \quad \text { where } \quad\langle x, y\rangle:=\frac{2(x, y)}{(y, y)} .
$$

Note that the inner product is invariant under the action of the Weyl group. Identifying $G L(E)$ to $G L(4, \mathbb{R})$ by means of the matrices relative to the $\mathbb{R}$-basis $\Delta$, the reflections $s_{i}$ are represented by

$$
\begin{array}{ll}
s_{1}=\left(\begin{array}{cccc}
-1 & 0 & 0 & 0 \\
1 & 1 & 0 & 0 \\
0 & 0 & 1 & 0 \\
0 & 0 & 0 & 1
\end{array}\right), \quad s_{2}=\left(\begin{array}{cccc}
1 & 1 & 0 & 0 \\
0 & -1 & 0 & 0 \\
0 & 1 & 1 & 0 \\
0 & 0 & 0 & 1
\end{array}\right), \\
s_{3}=\left(\begin{array}{cccc}
1 & 0 & 0 & 0 \\
0 & 1 & 2 & 0 \\
0 & 0 & -1 & 0 \\
0 & 0 & 1 & 1
\end{array}\right), \quad s_{4}=\left(\begin{array}{cccc}
1 & 0 & 0 & 0 \\
0 & 1 & 0 & 0 \\
0 & 0 & 1 & 1 \\
0 & 0 & 0 & -1
\end{array}\right),
\end{array}
$$

since the Cartan integers $\left\langle\alpha_{i}, \alpha_{j}\right\rangle$ are just the entries of the Cartan matrix.

We shall consider $\mathcal{W} \subset G L(4, \mathbb{R})$ ordered lexicographically, that is, first for any two different couples $(i, j),(k, l)$ such that $i, j, k, l \in\{1,2,3,4\}$ we define $(i, j)<(k, l)$ if and only if either $i<k$ or $i=k$ and $j<l$, and second, for any two different matrices $\sigma=\left(\sigma_{i j}\right), \sigma^{\prime}=\left(\sigma_{i j}^{\prime}\right)$ in $\mathcal{W}, \sigma<\sigma^{\prime}$ if and only if $\sigma_{i j}<\sigma_{i j}^{\prime}$ where $(i, j)$ is the least element (with the previous order in the couples) such that $\sigma_{i j} \neq \sigma_{i j}^{\prime}$. One possible way to compute the Weyl group with this particular enumeration is provided by the following code implemented with Mathematica:

$$
\begin{aligned}
& \mathrm{W}=\text { Table }\left[s_{i},\{\mathrm{i}, 4\}\right] ; \\
& \begin{array}{r}
\mathrm{a}\left[\mathrm{L}_{-}, \mathrm{x}_{-}\right]:=\text {Union }[\mathrm{L}, \\
\text { Table }[\mathrm{L}[\mathrm{i}]] . \mathrm{x},\{\mathrm{i}, \text { Length }[\mathrm{L}]\}], \\
\text { Table }[\mathrm{x} . \mathrm{L}[[\mathrm{i}]],\{\mathrm{i}, \text { Length }[\mathrm{L}]\}]]
\end{array} \\
& \begin{aligned}
\text { Do }\left[\mathrm{W}=\mathrm{a}\left[\mathrm{W}, s_{i}\right],\{\mathrm{i}, 4\}\right] \quad \text { (times repeated) }
\end{aligned}
\end{aligned}
$$

We get a list of $1152=2^{7} 3^{2}$ elements in the table $\mathrm{W}$ which is nothing but the Weyl group $\mathcal{W}$ of $\mathfrak{f}_{4}$. We are denoting by $\sigma_{i}$ the $i$-th element of $\mathcal{W}$ lexicographically ordered. The following result comes from a straightforward computation which may be done with any matrix multiplication software. 
Proposition 3. The 1152 elements of the Weyl group $\mathcal{W}$ of $\mathfrak{f}_{4}$ are distributed in 25 orbits (=conjugacy classes) according to the following table

\begin{tabular}{|c|c|c|c|}
\hline order & no. of elements & no. of orbits & representatives \\
\hline 1 & 1 & 1 & $\sigma_{748}=1$ \\
2 & 139 & 7 & $\sigma_{28}, \sigma_{42}, \sigma_{55}, \sigma_{103}$, \\
& & & $\sigma_{105}, \sigma_{142}, \sigma_{405}$ \\
3 & 80 & 3 & $\sigma_{7}, \sigma_{15}, \sigma_{114}$ \\
4 & 228 & 5 & $\sigma_{1}, \sigma_{3}, \sigma_{56}$, \\
& & & $\sigma_{104}, \sigma_{110}$ \\
6 & 464 & 7 & $\sigma_{4}, \sigma_{8}, \sigma_{9}, \sigma_{14}$, \\
8 & 144 & 1 & $\sigma_{30}, \sigma_{78}, \sigma_{106}$ \\
12 & 96 & 1 & $\sigma_{2}$ \\
\hline & 1152 & 25 & $\sigma_{10}$ \\
\hline
\end{tabular}

The column in the left gives the order of every element in the corresponding orbit. We shall denote by $I$ the set of indices of representatives in the right column:

$$
\begin{aligned}
& 1,2,3,4,7,8,9,10,14,15,28,30,42,55,56,78,103,104,105,106 \\
& 110,114,142,405,748 .
\end{aligned}
$$

For more information about the different orbits and representatives in terms of simple reflections, see the final paragraphs in the Appendix.

\subsection{The maximal torus of aut $\left(\mathfrak{f}_{4}\right)$}

If $\mathfrak{h}$ is a Cartan subalgebra of $L=\mathfrak{f}_{4}$, consider $L=\mathfrak{h} \oplus\left(\oplus_{\alpha \in \mathfrak{h}^{*}} L_{\alpha}\right)$ the decomposition in root spaces relative to $\mathfrak{h}$, that is, $L_{\alpha}=\{x \in L \mid[h, x]=$ $\alpha(h) x \forall h \in \mathfrak{h}\}$ if $\alpha \in \mathfrak{h}^{*}, \Phi=\left\{\alpha \in \mathfrak{h}^{*} \mid L_{\alpha} \neq 0\right\}$ the root system, and take a basis $\Delta=\left\{\alpha_{1}, \alpha_{2}, \alpha_{3}, \alpha_{4}\right\}$ of the root system. Identifying the roots to their coordinates relative to the basis $\Delta$, the 24 positive roots of $\Phi^{+}$are:

$$
\begin{array}{lll}
(0,0,0,1), & (0,1,1,1), & (1,2,2,1), \\
(0,0,1,0), & (0,1,2,0), & (1,1,2,2), \\
(0,1,0,0), & (1,1,1,1), & (1,2,3,1), \\
(1,0,0,0), & (0,1,2,1), & (1,2,2,2), \\
(0,0,1,1), & (1,1,2,0), & (1,2,3,2), \\
(0,1,1,0), & (1,1,2,1), & (1,2,4,2), \\
(1,1,0,0), & (0,1,2,2), & (1,3,4,2), \\
(1,1,1,0), & (1,2,2,0), & (2,3,4,2) .
\end{array}
$$


As usual, the nondegeneracy of the Killing form $k$ allows to identify $\mathfrak{h}$ to $\mathfrak{h}^{*}$, calling $t_{\alpha}$ the unique element in $\mathfrak{h}$ satisfying $\alpha(h)=k\left(t_{\alpha}, h\right)$ for all $h \in \mathfrak{h}$, as in $[25$, p. 37] (in this way the above mentioned inner product $($,$) in E$ is $\left.(\alpha, \beta)=k\left(t_{\alpha}, t_{\beta}\right)\right)$.

Any automorphism fixing pointwise $\mathfrak{h}$ preserves the root spaces. The set of all such automorphisms is a maximal torus of aut $(L)$; more precisely, given $x, y, z, u \in F^{\times}$there is a unique automorphism $\Psi$ such that

$$
\left.\Psi\right|_{\mathfrak{h}}=\mathrm{id},\left.\quad \Psi\right|_{L_{\alpha_{1}}}=x \mathrm{id},\left.\quad \Psi\right|_{L_{\alpha_{2}}}=y \mathrm{id},\left.\quad \Psi\right|_{L_{\alpha_{3}}}=z \mathrm{id},\left.\quad \Psi\right|_{L_{\alpha_{4}}}=u \mathrm{id}
$$

(particular case of the isomorphism theorem in [25, p.75]). Obviously, if $\alpha=n_{1} \alpha_{1}+n_{2} \alpha_{2}+n_{3} \alpha_{3}+n_{4} \alpha_{4}$, then $\left.\Psi\right|_{L_{\alpha}}=x^{n_{1}} y^{n_{2}} z^{n_{3}} u^{n_{4}}$ id. Denote by $\Psi_{x y z u}$ the above automorphism $\Psi$, and by $T_{\mathfrak{h}}$ the maximal torus $\left\{\Psi_{x y z u} \mid\right.$ $\left.x, y, z, u \in F^{\times}\right\}\left(T_{\mathfrak{h}}\right.$ depends only on $\mathfrak{h}$, and $\Psi_{x y z u}$ depends on $\mathfrak{h}$ and $\left.\Delta\right)$.

On the other hand, we have got a concrete maximal torus of $\mathfrak{F}_{4}$, since we have got an algebraic group isomorphism Ad: $F_{4} \rightarrow \mathfrak{F}_{4}$ and we have already introduced the maximal torus $\mathfrak{T}_{0}$ of $F_{4}$ (see (2.2)). Thus we get a maximal torus $\mathfrak{T}:=\operatorname{Ad}\left(\mathfrak{T}_{0}\right)$ in $\mathfrak{F}_{4}$, whose generic element is $t_{x y z u}^{\prime}:=\operatorname{Ad}\left(t_{x y z u}\right)$. Let us take as $\mathfrak{h}$ the Cartan subalgebra of the elements fixed by $\mathfrak{T}$. Since $\mathfrak{T}$ is contained in $T_{\mathfrak{h}}$, they necessarily coincide.

Let us choose a comfortable basis of $\mathfrak{f}_{4}$ for which we know the matrix representation of $t_{x y z u}^{\prime}$ relative to it. Let $\omega_{i}(i \in\{1, \ldots, 27\})$ be the $i$-th element in $B$, the standard basis on $J$ that we chose in Subsection 2.2. Recall that $t_{x y z u}\left(\omega_{i}\right)=\eta_{i} \omega_{i}$ where $\eta_{i}\left(=\eta_{i}(x, y, z, u)\right)$ is the $i$-th entry of the vector

$$
\begin{gathered}
\left(1,1,1, x, \frac{1}{x}, y, z, \frac{u^{2}}{x y z}, \frac{1}{y}, \frac{1}{z}, \frac{x y z}{u^{2}}, u, \frac{1}{u}, \frac{x y}{u}, \frac{x z}{u},\right. \\
\left.\frac{u}{y z}, \frac{u}{x y}, \frac{u}{x z}, \frac{y z}{u}, \frac{u}{x}, \frac{x}{u}, \frac{y}{u}, \frac{z}{u}, \frac{u}{x y z}, \frac{u}{y}, \frac{u}{z}, \frac{x y z}{u}\right) .
\end{gathered}
$$

For any $v \in J$ define the map $R_{v}: J \rightarrow J$ such that $a \mapsto a v$. Since $\mathfrak{f}_{4}=\operatorname{Der}(J)=\left[R_{J}, R_{J}\right]([39$, p. 117$])$, we can extract a basis of $\mathfrak{f}_{4}$ from the generators set $\left\{\left[R_{\omega_{i}}, R_{\omega_{j}}\right]\right\}_{i, j=1}^{27}$. Taking into account that

$$
\operatorname{Ad}\left(t_{x y z u}\right)\left[R_{\omega_{i}}, R_{\omega_{j}}\right]=\left[R_{t_{x y z u}\left(\omega_{i}\right)}, R_{t_{x y z u}\left(\omega_{j}\right)}\right]=\eta_{i} \eta_{j}\left[R_{\omega_{i}}, R_{\omega_{j}}\right]
$$

and defining $S$ as the set of all couples $(i, j) \in\{1, \ldots, 27\}^{2}$ such that $\left[R_{\omega_{i}}, R_{\omega_{j}}\right] \neq 0$, we have that the eigenvalues of $t_{x y z u}^{\prime}=\operatorname{Ad}\left(t_{x y z u}\right)$ are those 
of the set $\left\{\eta_{i} \eta_{j} \mid(i, j) \in S\right\}$, which are precisely:

$$
\begin{array}{r}
\left(1,1,1,1, x, \frac{1}{u}, y z, \frac{u^{2}}{x y^{2} z}, \frac{x}{u}, \frac{y z}{u}, \frac{u^{2}}{x y}, \frac{u}{x y}, \frac{x y z}{u}, \frac{y z}{u^{2}}, \frac{u}{y}, \frac{x y z}{u^{2}}, \frac{1}{x y}, \frac{1}{y}, \frac{x^{2} y z}{u^{2}},\right. \\
\frac{z}{x}, z, \frac{x}{y}, \frac{z}{u}, x z, \frac{x z}{u}, \frac{x z}{u^{2}}, \frac{x y z^{2}}{u^{2}}, \frac{z}{y}, \frac{1}{x}, u, \frac{1}{y z}, \frac{x y^{2} z}{u^{2}}, \frac{u}{x}, \frac{u}{y z}, \frac{x y}{u^{2}}, \frac{x y}{u}, \frac{u}{x y z}, \\
\text { (4.4) } \left.\frac{u^{2}}{y z}, \frac{y}{u}, \frac{u^{2}}{x y z}, x y, y, \frac{u^{2}}{x^{2} y z}, \frac{x}{z}, \frac{1}{z}, \frac{y}{x}, \frac{u}{z}, \frac{1}{x z}, \frac{u}{x z}, \frac{u^{2}}{x z}, \frac{u^{2}}{x y z^{2}}, \frac{y}{z}\right),
\end{array}
$$

where each eigenvalue is repeated according to its multiplicity (looking only at $S$, we would not know the multiplicities because the set $\left\{\eta_{i} \eta_{j} \mid(i, j) \in S\right\}$ has 228 elements, but 1 must appear 4 times, and the remaining values at least once, so by dimension count those are just the multiplicities). On the other hand, recalling that $T_{\mathfrak{h}}=\mathfrak{T}$, there must exist rational functions $X, Y, Z, U \in F(x, y, z, u)$ in the list (4.4) such that the whole list agrees with $\left(1,1,1,1, X^{n_{1}} Y^{n_{2}} Z^{n_{3}} U^{n_{4}} \mid\left(n_{1}, n_{2}, n_{3}, n_{4}\right) \in \Phi\right)$, with $\Phi=\Phi^{+} \cup\left(-\Phi^{+}\right)$ and $\Phi^{+}$given by (4.3). One solution is, for instance

$$
X=\frac{u^{2}}{x y^{2} z}, \quad Y=y z, \quad Z=\frac{1}{u}, \quad U=x .
$$

Next we choose as our reference basis of $\mathfrak{f}_{4}$ anyone extracted from

$$
\left\{\left[R_{\omega_{i}}, R_{\omega_{j}}\right]\right\}_{(i, j) \in S}
$$

such that the matrix of $t_{x y z u}^{\prime}$ relative to this basis is diagonal with the list (4.4) as diagonal. One possible choice is

$$
\begin{array}{llll}
b_{1}=\left[R_{\omega_{1}}, R_{\omega_{4}}\right] & b_{2}=\left[R_{\omega_{1}}, R_{\omega_{13}}\right] & b_{3}=\left[R_{\omega_{6}}, R_{\omega_{7}}\right] & b_{4}=\left[R_{\omega_{8}}, R_{\omega_{9}}\right] \\
b_{5}=\left[R_{\omega_{2}}, R_{\omega_{21}}\right] & b_{6}=\left[R_{\omega_{1}}, R_{\omega_{19}}\right] & b_{7}=\left[R_{\omega_{7}}, R_{\omega_{8}}\right] & b_{8}=\left[R_{\omega_{1}}, R_{\omega_{17}}\right] \\
b_{9}=\left[R_{\omega_{2}}, R_{\omega_{27}}\right] & b_{10}=\left[R_{\omega_{5}}, R_{\omega_{11}}\right] & b_{11}=\left[R_{\omega_{2}}, R_{\omega_{25}}\right] & b_{12}=\left[R_{\omega_{1}}, R_{\omega_{11}}\right] \\
b_{13}=\left[R_{\omega_{5}}, R_{\omega_{9}}\right] & b_{14}=\left[R_{\omega_{1}}, R_{\omega_{9}}\right] & b_{15}=\left[R_{\omega_{4}}, R_{\omega_{11}}\right] & b_{16}=\left[R_{\omega_{5}}, R_{\omega_{7}}\right] \\
b_{17}=\left[R_{\omega_{1}}, R_{\omega_{7}}\right] & b_{18}=\left[R_{\omega_{4}}, R_{\omega_{9}}\right] & b_{19}=\left[R_{\omega_{2}}, R_{\omega_{23}}\right] & b_{20}=\left[R_{\omega_{4}}, R_{\omega_{7}}\right] \\
b_{21}=\left[R_{\omega_{1}}, R_{\omega_{15}}\right] & b_{22}=\left[R_{\omega_{9}}, R_{\omega_{11}}\right] & b_{23}=\left[R_{\omega_{7}}, R_{\omega_{11}}\right] & b_{24}=\left[R_{\omega_{7}}, R_{\omega_{9}}\right] \\
b_{25}=\left[R_{\omega_{1}}, R_{\omega_{5}}\right] & b_{26}=\left[R_{\omega_{1}}, R_{\omega_{12}}\right] & b_{27}=\left[R_{\omega_{9}}, R_{\omega_{10}}\right] & b_{28}=\left[R_{\omega_{6}}, R_{\omega_{11}}\right] \\
b_{29}=\left[R_{\omega_{2}}, R_{\omega_{20}}\right] & b_{30}=\left[R_{\omega_{1}}, R_{\omega_{16}}\right] & b_{31}=\left[R_{\omega_{10}}, R_{\omega_{11}}\right] & b_{32}=\left[R_{\omega_{1}}, R_{\omega_{14}}\right] \\
b_{33}=\left[R_{\omega_{2}}, R_{\omega_{24}}\right] & b_{34}=\left[R_{\omega_{4}}, R_{\omega_{8}}\right] & b_{35}=\left[R_{\omega_{2}}, R_{\omega_{22}}\right] & b_{36}=\left[R_{\omega_{1}}, R_{\omega_{8}}\right] \\
b_{37}=\left[R_{\omega_{4}}, R_{\omega_{6}}\right] & b_{38}=\left[R_{\omega_{1}}, R_{\omega_{6}}\right] & b_{39}=\left[R_{\omega_{5}}, R_{\omega_{8}}\right] & b_{40}=\left[R_{\omega_{4}}, R_{\omega_{10}}\right] \\
b_{41}=\left[R_{\omega_{1}}, R_{\omega_{10}}\right] & b_{42}=\left[R_{\omega_{5}}, R_{\omega_{6}}\right] & b_{43}=\left[R_{\omega_{2}}, R_{\omega_{26}}\right] & b_{44}=\left[R_{\omega_{5}}, R_{\omega_{10}}\right] \\
b_{45}=\left[R_{\omega_{1}}, R_{\omega_{18}}\right] & b_{46}=\left[R_{\omega_{6}}, R_{\omega_{8}}\right], & b_{47}=\left[R_{\omega_{8}}, R_{\omega_{10}}\right] & b_{48}=\left[R_{\omega_{6}}, R_{\omega_{10}}\right]
\end{array}
$$

where these are root vectors relative to $\mathfrak{h}$, but the missing elements spanning the Cartan subalgebra must be taken carefully. If we denote $\beta_{i} \in \mathfrak{h}^{*}$ for $i=1 \ldots 48$ such that $\left[h, b_{i}\right]=\beta_{i}(h) b_{i}$ for any $h \in \mathfrak{h}$, it is easy to check that 
$\left(\beta_{4}, \beta_{3}, \beta_{2}, \beta_{1}\right)$ is a basis of $\Phi$ with Cartan matrix (4.1), and this will be our selection for $\Delta$ from now on (and respectively for $\alpha_{i}$ ). Thus, we can denote

$$
\begin{array}{lll}
b_{1}=v_{\alpha_{4}} & b_{25}=v_{-\alpha_{4}} \\
b_{2}=v_{\alpha_{3}} & b_{26}=v_{-\alpha_{3}} \\
b_{3}=v_{\alpha_{2}} & b_{27}=v_{-\alpha_{2}} \\
b_{4}=v_{\alpha_{1}} & b_{28}=v_{-\alpha_{1}} \\
b_{5}=v_{\alpha_{3}+\alpha_{4}} & b_{29}=v_{-\alpha_{3}-\alpha_{4}} \\
b_{6}=v_{\alpha_{2}+\alpha_{3}} & b_{31}=v_{-\alpha_{2}-\alpha_{3}} \\
b_{7}=v_{\alpha_{1}+\alpha_{2}} & b_{32}=v_{-\alpha_{1}-\alpha_{2}-\alpha_{3}} \\
b_{8}=v_{\alpha_{1}+\alpha_{2}+\alpha_{3}} & b_{33}=v_{-\alpha_{2}-\alpha_{3}-\alpha_{4}} \\
b_{9}=v_{\alpha_{2}+\alpha_{3}+\alpha_{4}} & b_{34}=v_{-\alpha_{2}-2 \alpha_{3}} \\
b_{10}=v_{\alpha_{2}+2 \alpha_{3}} & b_{35}=v_{-\alpha_{1}-\alpha_{2}-\alpha_{3}-\alpha_{4}} \\
b_{11}=v_{\alpha_{1}+\alpha_{2}+\alpha_{3}+\alpha_{4}} & b_{36}=v_{-\alpha_{2}-2 \alpha_{3}-\alpha_{4}} \\
b_{12}=v_{\alpha_{2}+2 \alpha_{3}+\alpha_{4}} & b_{37}=v_{-\alpha_{1}-\alpha_{2}-2 \alpha_{3}} \\
b_{13}=v_{\alpha_{1}+\alpha_{2}+2 \alpha_{3}} & b_{38}=v_{-\alpha_{1}-\alpha_{2}-2 \alpha_{3}-\alpha_{4}} \\
b_{14}=v_{\alpha_{1}+\alpha_{2}+2 \alpha_{3}+\alpha_{4}} & b_{39}=v_{-\alpha_{2}-2 \alpha_{3}-2 \alpha_{4}} \\
b_{15}=v_{\alpha_{2}+2 \alpha_{3}+2 \alpha_{4}} & b_{40}=v_{-\alpha_{1}-2 \alpha_{2}-2 \alpha_{3}} \\
b_{16}=v_{\alpha_{1}+2 \alpha_{2}+2 \alpha_{3}} & b_{41}=v_{-\alpha_{1}-2 \alpha_{2}-2 \alpha_{3}-\alpha_{4}} \\
b_{17}=v_{\alpha_{1}+2 \alpha_{2}+2 \alpha_{3}+\alpha_{4}} & b_{42}=v_{-\alpha_{1}-\alpha_{2}-2 \alpha_{3}-2 \alpha_{4}} \\
b_{18}=v_{\alpha_{1}+\alpha_{2}+2 \alpha_{3}+2 \alpha_{4}} & b_{43}=v_{-\alpha_{1}-2 \alpha_{2}-3 \alpha_{3}-\alpha_{4}} \\
b_{19}=v_{\alpha_{1}+2 \alpha_{2}+3 \alpha_{3}+\alpha_{4}} & b_{44}=v_{-\alpha_{1}-2 \alpha_{2}-2 \alpha_{3}-2 \alpha_{4}} \\
b_{20}=v_{\alpha_{1}+2 \alpha_{2}+2 \alpha_{3}+2 \alpha_{4}} & b_{45}=v_{-\alpha_{1}-2 \alpha_{2}-3 \alpha_{3}-2 \alpha_{4}} \\
b_{21}=v_{\alpha_{1}+2 \alpha_{2}+3 \alpha_{3}+2 \alpha_{4}} & b_{46}=v_{-\alpha_{1}-2 \alpha_{2}-4 \alpha_{3}-2 \alpha_{4}} \\
b_{22}=v_{\alpha_{1}+2 \alpha_{2}+4 \alpha_{3}+2 \alpha_{4}} & b_{4} & b_{47}=v_{-\alpha_{1}-3 \alpha_{2}-4 \alpha_{3}-2 \alpha_{4}} \\
b_{23}=v_{\alpha_{1}+3 \alpha_{2}+4 \alpha_{3}+2 \alpha_{4}} & b_{48}=v_{-2 \alpha_{1}-3 \alpha_{2}-4 \alpha_{3}-2 \alpha_{4}} \\
b_{24}=v_{2 \alpha_{1}+3 \alpha_{2}+4 \alpha_{3}+2 \alpha_{4}} & b_{48} &
\end{array}
$$

where each $v_{\alpha}$ is a root vector relative to the root $\alpha$, verifying

$$
\operatorname{Ad}\left(t_{x y z u}\right) v_{m_{1} \alpha_{1}+m_{2} \alpha_{2}+m_{3} \alpha_{3}+m_{4} \alpha_{4}}=X^{m_{1}} Y^{m_{2}} Z^{m_{3}} U^{m_{4}} v_{m_{1} \alpha_{1}+m_{2} \alpha_{2}+m_{3} \alpha_{3}+m_{4} \alpha_{4}} .
$$

At last we choose our standard basis of $\mathfrak{f}_{4}$ as

$$
B^{\prime}=\left(4\left[b_{4}, b_{28}\right], 4\left[b_{27}, b_{3}\right], 8\left[b_{26}, b_{2}\right], 8\left[b_{25}, b_{1}\right], b_{i} \mid i=1, \ldots, 48\right),
$$

formed by root vectors which have been precisely described above. This will be needed in the next section to extend elements from $\mathcal{W}$ to $\mathfrak{F}_{4}$. The first four elements in $B^{\prime}$, which of course form a basis of $\mathfrak{h}$, have not been arbitrarily chosen, but they are respectively $t_{\alpha_{1}}, t_{\alpha_{2}}, t_{\alpha_{3}}$ and $t_{\alpha_{4}}$ for our election of $\Delta$.

Notice that we have algebraic group isomorphisms $\alpha, \beta:\left(F^{\times}\right)^{4} \rightarrow \mathfrak{T}$ such that $\alpha(x, y, z, u)=t_{x y z y u}^{\prime}=\operatorname{Ad}\left(t_{x y z y u}\right)$ and $\beta(X, Y, Z, U)=\Psi_{X Y Z U}$ (now we 
have fixed $\Delta)$. The map $\eta:(x, y, z, u) \mapsto(X, Y, Z, U)$ (see (4.5)) is an automorphism of the algebraic group $\left(F^{\times}\right)^{4}$ and we have a commutative diagram

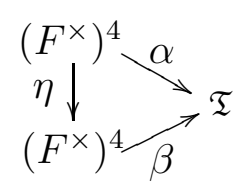

in which all the arrows are isomorphisms. But $\mathrm{Ad}: \mathfrak{T}_{0} \rightarrow \mathfrak{T}$ is also an isomorphism, which allows to write the simultaneous diagonalization of $J$ relative to $\mathfrak{T}_{0}$ as a $\mathbb{Z}^{4}$-grading in such a way that the induced grading on $\mathfrak{f}_{4}$ by Ad is just the root decomposition indexed in the coordinates of $\Phi$ relative to $\Delta$,

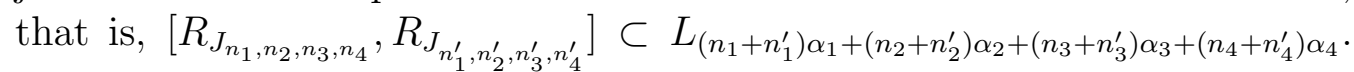
Let us explain this in a practical way: Since the set of eigenvalues of $t_{x y z u}$ is contained in the set of eigenvalues of $t_{x y z u}^{\prime}$, any eigenvalue of $t_{x y z u}$ can be written in the form $X^{m_{1}} Y^{m_{2}} Z^{m_{3}} U^{m_{4}}$. This defines a map from the set of eigenvalues of $t_{x y z u}$ to $\mathbb{Z}^{4}$ such that $X^{m_{1}} Y^{m_{2}} Z^{m_{3}} U^{m_{4}} \mapsto\left(m_{1}, m_{2}, m_{3}, m_{4}\right)$, providing a $\mathbb{Z}^{4}$-grading on the Albert algebra $J=\oplus J_{m_{1}, m_{2}, m_{3}, m_{4}}$ such that $\left(m_{1}, m_{2}, m_{3}, m_{4}\right)$ is in the image of the above map. To determine $J_{m_{1}, m_{2}, m_{3}, m_{4}}$ we find the eigenvalue of $t_{x y z u}$ of the form $X^{m_{1}} Y^{m_{2}} Z^{m_{3}} U^{m_{4}}$, and then take the element of the standard basis of the Albert algebra which is an eigenvector for that eigenvalue. For instance, to find $J_{1,2,2,1}$ we compute $X Y^{2} Z^{2} U=z$ and write $J_{1,2,2,1}=\left\langle u_{2}^{(3)}\right\rangle$ since $u_{2}^{(3)}$ is the basic element such that $t_{x y z u}\left(u_{2}^{(3)}\right)=z u_{2}^{(3)}$. The complete description of the grading is:

$$
\begin{array}{rlrl}
J_{0,0,0,0}=\left\langle E_{1}, E_{2}, E_{3}\right\rangle, & \\
J_{0,0,0,1}=\left\langle e_{1}^{(3)}\right\rangle, & J_{0,0,1,0}=\left\langle e_{1}^{(2)}\right\rangle, & & J_{0,0,-1,-1}=\left\langle e_{1}^{(1)}\right\rangle, \\
J_{0,0,0,-1}=\left\langle e_{2}^{(3)}\right\rangle, & J_{0,0,-1,0}=\left\langle e_{2}^{(2)}\right\rangle, & & J_{0,0,1,1}=\left\langle e_{2}^{(1)}\right\rangle, \\
J_{-1,-1,-2,-1}=\left\langle u_{1}^{(3)}\right\rangle, & J_{-1,-1,-1,0}=\left\langle u_{1}^{(2)}\right\rangle, & & J_{-1,-1,-1,-1}=\left\langle u_{1}^{(1)}\right\rangle, \\
J_{1,2,2,1}=\left\langle u_{2}^{(3)}\right\rangle, & J_{1,2,3,2}=\left\langle u_{2}^{(2)}\right\rangle, & & J_{1,2,3,1}=\left\langle u_{2}^{(1)}\right\rangle, \\
J_{0,-1,-2,-1}=\left\langle u_{3}^{(3)}\right\rangle, & J_{0,-1,-1,0}=\left\langle u_{3}^{(2)}\right\rangle, & & J_{0,-1,-1,-1}=\left\langle u_{3}^{(1)}\right\rangle, \\
J_{1,1,2,1}=\left\langle v_{1}^{(3)}\right\rangle, & J_{1,1,1,0}=\left\langle v_{1}^{(2)}\right\rangle, & & J_{1,1,1,1}=\left\langle v_{1}^{(1)}\right\rangle, \\
J_{-1,-2,-2,-1}=\left\langle v_{2}^{(3)}\right\rangle, & J_{-1,-2,-3,-2}=\left\langle v_{2}^{(2)}\right\rangle, & & J_{-1,-2,-3,-1}=\left\langle v_{2}^{(1)}\right\rangle, \\
J_{0,1,2,1}=\left\langle v_{3}^{(3)}\right\rangle, & J_{0,1,1,0}=\left\langle v_{3}^{(2)}\right\rangle, & & J_{0,1,1,1}=\left\langle v_{3}^{(1)}\right\rangle,
\end{array}
$$

which is of type $(24,0,1)$. We remark again that this $\mathbb{Z}^{4}$-grading on the Albert algebra is toral and fine and the induced grading on $\mathfrak{f}_{4}$ is precisely the Cartan grading. Alternatively we could have got this grading in Section 3 directly from the maximal torus of $\operatorname{aut}(J)$ but in that case its relationship to the Cartan grading would have not been so direct. The group $\mathbb{Z}^{4}$ is 
the universal group of the grading. Moreover all the toral gradings on the Albert algebra are coarsenings of this and so can be obtained by constructing equivalence classes of epimorphisms $\mathbb{Z}^{4} \rightarrow G$ modulo the relation given as in $[13,4.1]$. This is another way of understanding Proposition 1.

To finish this subsection we must devote a few lines to the action of the Weyl group $\mathcal{W}$ on the maximal torus of $\mathfrak{F}_{4}$. Since this is isomorphic to $\left(F^{\times}\right)^{4}$ we can introduce this action as the integration of the action of $\mathcal{W}$ on the Cartan subalgebra $\mathcal{W} \times \mathfrak{h} \rightarrow \mathfrak{h}$ such that $\sigma \cdot t_{\alpha}=t_{\sigma(\alpha)}$ for any $\sigma=\left(a_{i j}\right) \in \mathcal{W}$ and $\alpha \in \Phi$ ( $t_{\alpha}$ defined at the beginning of this section). Since $\sigma$ is an endomorphism of the dual space $\mathfrak{h}^{*}$, then the transposed matrix $\sigma^{t}$ represents the dual map $\mathfrak{h} \rightarrow \mathfrak{h}$. So identifying the elements in $\mathfrak{h}$ with their coordinates relative to the basis $\left(t_{\alpha_{i}}\right)_{i=1}^{4}$, the action of $\sigma$ on the element $\sum_{i} x_{i} t_{\alpha_{i}} \in \mathfrak{h}$ is given by $\left(x_{1}, x_{2}, x_{3}, x_{4}\right) \cdot\left[\left(a_{i j}\right)_{i, j=1}^{4}\right]^{t}$ where the product $\cdot$ is the usual matrix product. The integration of this is the desired action $\mathcal{W} \times\left(F^{\times}\right)^{4} \rightarrow\left(F^{\times}\right)^{4}$ which consequently acts in the form $\sigma \cdot(X, Y, Z, U)=\left(X^{\prime}, Y^{\prime}, Z^{\prime}, U^{\prime}\right)$ where

$$
\begin{aligned}
X^{\prime} & =X^{a_{11}} Y^{a_{12}} Z^{a_{13}} U^{a_{14}} \\
Y^{\prime} & =X^{a_{21}} Y^{a_{22}} Z^{a_{23}} U^{a_{24}} \\
Z^{\prime} & =X^{a_{31}} Y^{a_{32}} Z^{a_{33}} U^{a_{34}} \\
U^{\prime} & =X^{a_{41}} Y^{a_{42}} Z^{a_{43}} U^{a_{44}} .
\end{aligned}
$$

\subsection{Extending Weyl group elements to automorphisms of $\mathfrak{f}_{4}$}

In this subsection we shall use the isomorphism theorem of [25, p.75] for extending any $\sigma \in \mathcal{W}$ to an automorphism $\tilde{\sigma} \in \mathfrak{F}_{4}$. In the context of the mentioned theorem we can take $L=L^{\prime}=\mathfrak{f}_{4}, \mathfrak{h}=\mathfrak{h}^{\prime}$ agreeing with the Cartan subalgebra generated by the four first elements in $B^{\prime}$ the standard basis of $\mathfrak{f}_{4}, \Phi=\Phi^{\prime}$ the root system relative to $\mathfrak{h}$, we choose $\Delta=\Delta^{\prime}$ the basis of $\Phi$ as in $4.1\left(\alpha_{1}, \ldots \alpha_{4}\right.$ the roots corresponding to $b_{4}, \ldots b_{1} \in B^{\prime}$ respectively) and finally, we take as isomorphism $\mathfrak{h} \rightarrow \mathfrak{h}$ the induced by $\sigma$ (as above, by means of the identification $\mathfrak{h} \rightarrow \mathfrak{h}^{*}$ through $t \mapsto k(t,-)$ ). According to that theorem, for any choice $x_{\alpha_{i}} \in L_{\alpha_{i}} \backslash\{0\}$ and $x_{\sigma\left(\alpha_{i}\right)}^{\prime} \in$ $L_{\sigma\left(\alpha_{i}\right)} \backslash\{0\}$ for $i=1,2,3,4$, there is only one $\tilde{\sigma} \in \mathfrak{F}_{4}$ such that $\tilde{\sigma}\left(t_{\alpha_{i}}\right)=$ $t_{\sigma\left(\alpha_{i}\right)}$ and $\tilde{\sigma}\left(x_{\alpha_{i}}\right)=x_{\sigma\left(\alpha_{i}\right)}^{\prime}$ for every $i=1,2,3,4$. We choose $x_{\alpha_{i}}$ to be the generator $v_{\alpha_{i}} \in L_{\alpha_{i}}$ as in (4.6) and also $x_{\sigma\left(\alpha_{i}\right)}^{\prime}=v_{\sigma\left(\alpha_{i}\right)} \in L_{\sigma\left(\alpha_{i}\right)}$. The matrix of $\tilde{\sigma}$ relative to the standard basis is block diagonal $\left(\begin{array}{cc}A & 0 \\ 0 & D\end{array}\right)$ where $A$ is just the $4 \times 4$ matrix of $\sigma$ relative to the basis $\left\{\alpha_{i}\right\}_{i=1}^{4}$ and $D$ is a $48 \times 48$ matrix with only one nonzero element in each row and in each column. Thus we have constructed an injective map $\mathcal{W} \rightarrow \mathfrak{F}_{4}$ such that $\sigma \mapsto \tilde{\sigma}$ (see the Appendix for more information). It is important to highlight that this 
is not a group homomorphism but only a map. In fact, there does not exist a group monomorphism $\mathcal{W} \rightarrow \mathfrak{F}_{4}$, as it is proved in [33, p. 717].

We shall denote by $\mathfrak{N}$ the normalizer of $\mathfrak{T}$ in $\mathfrak{F}_{4}$. It is a standard result that $\mathcal{W} \cong \mathfrak{N} / \mathfrak{T}$. It follows easily, by construction of $\tilde{\sigma}$, that $\tilde{\sigma} \in \mathfrak{N}$ for any $\sigma \in \mathcal{W}$. Thus the previous map $\mathcal{W} \rightarrow \mathfrak{F}_{4}$ is actually a map $\mathcal{W} \rightarrow \mathfrak{N}$, and composing with the universal epimorphism $\mathfrak{N} \rightarrow \mathfrak{N} / \mathfrak{T}$ we get an injective map $\mathcal{W} \rightarrow \mathfrak{N} / \mathfrak{T}$ such that $\sigma \mapsto \tilde{\sigma} \mathfrak{T}$ (the equivalence class of $\tilde{\sigma}$ in the quotient group). Since domain and codomain of this map share the same finite cardinal, the map is a bijection. Even more: it can be proved that $\widetilde{\sigma_{1} \sigma_{2}}$ is in the same equivalence class that $\widetilde{\sigma_{1}} \widetilde{\sigma_{2}}$ (since $\widetilde{\sigma_{1} \sigma_{2}} \tilde{\sigma}_{2}^{-1} \tilde{\sigma}_{1}^{-1}$ acts in $\mathfrak{h}$ as $\sigma_{1} \sigma_{2} \sigma_{2}{ }^{-1} \sigma_{1}{ }^{-1}=\mathrm{id}$, so that it belongs to $\mathfrak{T}$ ) which proves that the previous map $\mathcal{W} \rightarrow \mathfrak{N} / \mathfrak{T}$ is a group isomorphism. In particular

$$
\mathfrak{N}=\{\tilde{\sigma} t \mid \sigma \in \mathcal{W}, t \in \mathfrak{T}\}
$$

We can now revisit the action of the Weyl group $\mathcal{W}$ on the maximal torus $\left(F^{\times}\right)^{4} \cong \mathfrak{T}$ from another viewpoint. Identifying $\mathcal{W}$ with $\mathfrak{N} / \mathfrak{T}$ we can define the action $\mathcal{W} \times \mathfrak{T} \rightarrow \mathfrak{T}$ given by $\sigma \cdot t:=\tilde{\sigma} t \tilde{\sigma}^{-1}$ for $\sigma \in \mathcal{W}$ and $t \in \mathfrak{T}$. Then the isomorphism $\beta:\left(F^{\times}\right)^{4} \rightarrow \mathfrak{T}$ given by $\beta(X, Y, Z, U)=\Psi_{X Y Z U}$ is an isomorphism of $\mathcal{W}$-groups in the sense that $\beta(\sigma \cdot t)=\sigma \cdot \beta(t)$. Thus $\sigma \cdot \Psi_{X Y Z U}=\Psi_{X^{\prime} Y^{\prime} Z^{\prime} U^{\prime}}$ as in (4.8). And since $\Psi_{X Y Z U}=t_{x y z u}^{\prime}$ for (4.5), a simple computation proves that $\sigma \cdot t_{x y z u}^{\prime}=t_{x^{\prime} y^{\prime} z^{\prime} u^{\prime}}^{\prime}$ where now

$$
\begin{aligned}
& x^{\prime}=x^{b_{11}} y^{b_{12}} z^{b_{13}} u^{b_{14}} \\
& y^{\prime}=x^{b_{21}} y^{b_{22}} z^{b_{23}} u^{b_{24}} \\
& z^{\prime}=x^{b_{31}} y^{b_{32}} z^{b_{33}} u^{b_{34}} \\
& u^{\prime}=x^{b_{41}} y^{b_{42}} z^{b_{43}} u^{b_{44}}
\end{aligned} \text { with }\left(b_{i j}\right)=m \sigma m^{-1}, m=\left(\begin{array}{cccc}
0 & 0 & 0 & 1 \\
-1 & -1 & -2 & -1 \\
1 & 2 & 2 & 1 \\
0 & 0 & -1 & 0
\end{array}\right) \text {. }
$$

The action of $\mathcal{W}$ on $\left(F^{\times}\right)^{4}$ given by $\sigma \cdot(x, y, z, u)=\left(x^{\prime}, y^{\prime}, z^{\prime}, u^{\prime}\right)$ as above is essential for our work, specially the study of fixed elements in the torus under the action of certain elements of $\mathcal{W}$. Denote

$$
\mathfrak{T}^{\langle j\rangle}=\left\{t \in \mathfrak{T} \mid \sigma_{j} \cdot t=t\right\}
$$

It is easily seen that this is a subgroup of $\mathfrak{T}$ such that $\mathfrak{T}^{\langle i\rangle} \cong \mathfrak{T}^{\langle j\rangle}$ when $\sigma_{i}$ is conjugated to $\sigma_{j}$ in $\mathcal{W}$. The information given by these subgroups $\mathfrak{T}^{\langle i\rangle}$ is needed for our purposes, so we are calculating them. For this, it suffices to consider the representatives of conjugacy classes given in the table of Proposition 3. We summarize all this information in the following table. In it, $\mathfrak{T}^{\langle j\rangle}$ is the subgroup of all $t_{x y z u}^{\prime} \in \mathfrak{T}$ such that the element given satisfies the displayed condition. We also write down the abstract group isomorphic to $\mathfrak{T}^{\langle j\rangle}$ in the right column. 


\begin{tabular}{|c|c|c|c|}
\hline$j$ & Generic element of $\mathfrak{T}^{\langle j\rangle}$ & Membership condition & Isomorphic to \\
\hline 1 & $\left(u^{2} y, y, y, u\right)$ & $y^{2}=1, u \in F^{\times}$ & $F^{\times} \times \mathbb{Z}_{2}$ \\
2 & $(x, x, x, 1)$ & $x^{2}=1$ & $\mathbb{Z}_{2}$ \\
3 & $\left(u^{2} y, y, u^{2}, u\right)$ & $u^{4}=1=y^{2}$ & $\mathbb{Z}_{4} \times \mathbb{Z}_{2}$ \\
4 & $\left(x, x^{-1}, 1, x^{-1}\right)$ & $x \in F^{\times}$ & $F^{\times}$ \\
7 & $\left(u^{2} y^{-3}, y, y, u\right)$ & $u, y \in F^{\times}$ & $\left(F^{\times}\right)^{2}$ \\
8 & $\left(x, x, x, x^{2}\right)$ & $x \in F^{\times}$ & $F^{\times}$ \\
9 & $\left(1, u^{2 / 3}, u^{2 / 3}, u\right)$ & $u \in F^{\times}$ & $F^{\times}$ \\
10 & $(1,1,1,1)$ & & $\{1\}$ \\
14 & $(x, x, x, y)$ & $x^{2}=y^{2}=1$ & $\mathbb{Z}_{2}^{2}$ \\
15 & $\left(x, y, x^{2} y, x^{2}\right)$ & $x^{3}=y^{3}=1$ & $\mathbb{Z}_{3}^{2}$ \\
28 & $\left(x, x^{-1}, z, x^{-1}\right)$ & $x, z \in F^{\times}$ & $F^{\times}$ \\
30 & $(1,1, z, 1)$ & $z \in F^{\times}$ & $F^{\times} \times \mathbb{Z}_{2}^{2}$ \\
42 & $(x, x, z, u)$ & $x, y, u \in F^{\times}$ & $\left(F^{\times}\right)^{3}$ \\
55 & $(x, y, y, u)$ & $x, y \in F^{\times}$ & $\left(F^{\times}\right)^{2}$ \\
56 & $(x, y, y, x y)$ & & $\{1\}$ \\
78 & $(1,1,1,1)$ & $u^{2}=1, x, u \in F^{\times}$ & $\left(F^{\times}\right)^{2} \times \mathbb{Z}_{2}$ \\
103 & $(x, y, y, u)$ & $y^{2}=1, x \in F^{\times}$ & $F^{\times} \times \mathbb{Z}_{2}$ \\
104 & $(x, y, y, x y)$ & $x^{2}=y^{2}=1, u \in F^{\times}$ & $F^{\times} \times \mathbb{Z}_{2}^{2}$ \\
105 & $(x, y, x y, u)$ & $x^{2}=y^{2}=1$ & $\mathbb{Z}_{2}^{2}$ \\
106 & $(x, y, x y, y)$ & $x^{2}=u^{2}=1$ & $\mathbb{Z}_{2}^{2}$ \\
110 & $(x, x, x, u)$ & $x, z \in F^{\times}$ & $\left(F^{\times}\right)^{2}$ \\
114 & $(x, 1, z, 1)$ & $x, z, u \in F^{\times}$ & $\left(F^{\times}\right)^{3}$ \\
142 & $(x, 1, z, u)$ & $x, y, z, u \in F^{\times}$ & $\left(F_{2}^{\times}\right)^{4}$ \\
405 & $(x, y, z, u)$ & $x^{2}=z^{2}=u^{2}=1$ & \\
748 & $(x, y, z, u)$ & & \\
\hline
\end{tabular}

\section{Quasitori in $F_{4}$}

Recall that a quasitorus is a commutative algebraic group whose identity component is a torus [34, p. 105]. An algebraic linear group is a quasitorus if and only if in some basis its elements can be expressed simultaneously by diagonal matrices. Such groups are also called diagonalizable. Besides, a quasitorus $Q$ in an algebraic group $G$ can be written as a disjoint union $Q=T \cup T a_{1} \cup \cdots \cup T a_{k}$ where $T$ is a torus and $\left\{1_{G}, a_{1}, \ldots, a_{k}\right\}$ a finite abelian subgroup of $G$. We remark that, as a consequence of the algebraic version of the Borel-Serre theorem (Theorem 6 in the Appendix), any quasitorus in $G$ normalizes some of the maximal tori of $G$. To see that, define $Z$ as the centralizer in $G$ of $T$. Applying this theorem to $H:=\left\{1_{G}, a_{1}, \ldots, a_{k}\right\}$, which is contained in $Z$, there is some maximal torus $T^{\prime}$ of $Z$ such that $H \subset \mathfrak{N}_{Z}\left(T^{\prime}\right)$ (the normalizer of $T^{\prime}$ in $Z$ ). But $T$ is contained in the center of $Z$ and since all its elements are semisimple, $T$ is contained in the intersection of all maximal tori of $Z$, hence in $T^{\prime}$. Thus $T \subset T^{\prime} \subset \mathfrak{N}_{Z}\left(T^{\prime}\right)$ and since we had $H \subset \mathfrak{N}_{Z}\left(T^{\prime}\right) \subset \mathfrak{N}_{G}\left(T^{\prime}\right)$ then $Q \subset \mathfrak{N}_{G}\left(T^{\prime}\right)$. But actually $T^{\prime}$ is a maximal torus in $G$, because if $T^{\prime \prime}$ is a maximal torus of $G$ which contains $T^{\prime} \supset T$, then $T^{\prime \prime} \subset Z$ and $T^{\prime \prime}=T^{\prime}$. 
As any grading is given by a quasitorus, the above paragraph gives the reason why our arguments are usually related to facts occurring inside $\mathfrak{N}=\mathcal{W} \mathfrak{T}$. As a consequence we have studied in detail the Weyl group and its action on the torus $\mathfrak{T}$ in the previous section.

Next we consider a class of quasitori which is relevant for our study. Define for each $j \in\{1, \ldots, 1152\}$ and each $t \in \mathfrak{T}$ the quasitorus $A(j, t)$ as the (closed) subgroup of $\mathfrak{F}_{4}$ generated by $\mathfrak{T}^{\langle j\rangle}$ and $\widetilde{\sigma}_{j} t$. This defines of course a grading on $\mathfrak{f}_{4}$ by the group $\mathfrak{X}(A(j, t))$ as in 2.1. But it suffices to consider the gradings induced by the quasitori $A(j, t)$ with $j \in I$ (the set $I$ defined in (4.2)), taking into account that if $\sigma_{i}$ and $\sigma_{j}$ are conjugated in $\mathcal{W}$, then $A(i, t) \cong A\left(j, t^{\prime}\right)$ for a suitable $t^{\prime} \in \mathfrak{T}$. We also have

Proposition 4. If for some $j$ the group $A(j, i d)$ is toral then $A(j, t)$ is toral for any $t \in \mathfrak{T}$.

Proof. Let $Z=\mathfrak{C}_{\mathfrak{F}_{4}}\left(\mathfrak{T}^{\langle j\rangle}\right)$ and $Z_{0}$ its unit component. Since $A(j$,id) is toral there is some maximal torus $T$ of $\mathfrak{F}_{4}$ such that $A(j$, id $) \subset T$. Then $T \subset Z$ but from $\mathfrak{T}^{\langle j\rangle} \subset \mathfrak{T}$ we also get $\mathfrak{T} \subset Z$. Of course $\mathfrak{T}, T \subset Z_{0}$ and since $t \in \mathfrak{T}$ and $\widetilde{\sigma}_{j} \in A(j$, id $) \subset T$ we have $\widetilde{\sigma}_{j}, t \in Z_{0}$ hence $\widetilde{\sigma}_{j} t \in Z_{0}$. But $\widetilde{\sigma}_{j} t$ is a semisimple element of $Z_{0}$ and consequently there is some $p \in Z_{0}$ such that $p \widetilde{\sigma}_{j} t p^{-1} \in \mathfrak{T}$. This jointly with the fact that $p \mathfrak{T}^{\langle j\rangle} p^{-1}=\mathfrak{T}^{\langle j\rangle}$ imply that $p A(j, t) p^{-1} \subset \mathfrak{T}$.

The same proof shows that $A(j, t)$ is toral if and only if $A\left(j, t^{\prime}\right)$ is toral for all $t^{\prime} \in \mathfrak{T}$. Now let us detect the indices which cause nontorality.

Proposition 5. For $j \in I$ the group $A(j, i d)$ is nontoral if and only if $j=3,15,105,106$ or 405 .

Proof. Let us prove first that the five quasitori are nontoral. For $A(15$,id) we have $\mathfrak{T}^{\langle 15\rangle} \cong \mathbb{Z}_{3}^{2}$ (see Table of Section 4.2 ). The grading induced by this quasitorus is produced by the automorphisms $\left\{t_{\omega, \omega, 1, \omega^{2}}^{\prime}, t_{1, \omega, \omega, 1}^{\prime}, \widetilde{\sigma_{15}}\right\}$ where $\omega$ is a primitive cubic root of 1 . This is a $\mathbb{Z}_{3}^{3}$-grading and computing the subalgebra of fixed elements by the three previous automorphisms we find that this is null. This implies that the grading is nontoral since in the toral case, this should be an algebra of rank four. For $A(405$, id) we have $\mathfrak{T}^{\langle 405\rangle} \cong \mathbb{Z}_{2}^{4}$ and the associated grading agrees with the one produced by $\left\{t_{-1,1,1,1}^{\prime}, t_{1,-1,1,1}^{\prime}, t_{1,1,-1,1}^{\prime}, t_{1,1,1,-1}^{\prime}, \widetilde{\sigma_{405}}\right\}$. This is a $\mathbb{Z}_{2}^{5}$-grading whose 0 homogeneous component is again null, hence the grading is nontoral. For $A(3$, id $)$ we have $\mathfrak{T}^{\langle 3\rangle} \cong \mathbb{Z}_{4} \times \mathbb{Z}_{2}$ and the induced grading is the produced by $\left\{t_{1,-1,-1, i}^{\prime}, t_{-1,-1,1,1}^{\prime}, \widetilde{\sigma}_{3}\right\}$ which is a $\mathbb{Z}_{2} \times \mathbb{Z}_{2} \times \mathbb{Z}_{8}$-grading (see Remarks 1 and 2 after this proof) whose 0 -homogeneous component has dimension 1 . Hence $A(3, \mathrm{id})$ is nontoral. The grading induced by $A(106, \mathrm{id})$ is also nontoral since $\mathfrak{T}^{\langle 106\rangle} \cong \mathbb{Z}_{2}^{2}$ and the grading is the one induced by $\left\{t_{-1,1,-1,1}^{\prime}, t_{1,-1,-1,-1}^{\prime}, \widetilde{\sigma_{106}}\right\}$, 
which is a $\mathbb{Z}_{2}^{2} \times \mathbb{Z}_{6}$-grading whose 0 -homogeneous component is one-dimensional. The last grading is the induced by $A\left(105\right.$,id). We have $\mathfrak{T}^{\langle 105\rangle} \cong$ $F^{\times} \times \mathbb{Z}_{2}^{2}$, so $A(105$, id $)=\left\langle\mathfrak{T}^{\langle 105\rangle}, \widetilde{\sigma}_{105}\right\rangle \cong F^{\times} \times \mathbb{Z}_{2}^{3}$ which induces a grading over $\mathfrak{X}\left(F^{\times} \times \mathbb{Z}_{2}^{3}\right) \cong \mathbb{Z} \times \mathbb{Z}_{2}^{3}$. The grading agrees with the one produced for instance by $\left\{\widetilde{\sigma_{105}}, t_{x, y, x y, 1}^{\prime}, t_{1,1,1,2}^{\prime} \mid x^{2}=y^{2}=1\right\}$, which can be implemented in a computer. This is a $\mathbb{Z}_{2}^{3} \times \mathbb{Z}$-grading whose 0 -homogeneous component is one-dimensional and so $A(105, \mathrm{id})$ is nontoral. We include also a table of homogeneous components dimensions for further reference. These types can be computed with any linear algebra software allowing simultaneous diagonalization.

\begin{tabular}{|c|c|}
\hline Quasitorus & Type \\
\hline$A(3, \mathrm{id})$ & $(19,6,7)$ \\
$A(15, \mathrm{id})$ & $(0,26)$ \\
$A(105, \mathrm{id})$ & $(31,0,7)$ \\
$A(106, \mathrm{id})$ & $(3,14,7)$ \\
$A(405, \mathrm{id})$ & $(24,0,0,7)$ \\
\hline
\end{tabular}

Let us prove now that $A(j, \mathrm{id})$ is toral in the rest of the cases. If $\mathfrak{T}^{\langle j\rangle}=\mathrm{id}$ then $A(j$, id) is cyclic and then toral (this applies to the cases $j=10,78)$. In case $\mathfrak{T}^{\langle j\rangle}$ is cyclic or $\mathfrak{T}^{\langle j\rangle} \cong F^{\times}$, then $A(j$, id) has two factors and by Lemma 2 ([2, Lemma 1.1.3, p. 5]) the grading is toral (this applies to $j=2,4,8,9,30$ ). Another trivial case is $j=748$ since $\widetilde{\sigma_{748}}=\mathrm{id}$ and $\mathfrak{T}^{\langle 748\rangle}=\mathfrak{T}$. For $j=1,7,14,28,42,55,56,103,104,114$ and 142 , performing a simultaneous diagonalization of the algebra relative to the set of automorphisms inducing the grading, one finds that the zero homogeneous component of the corresponding grading is an abelian four-dimensional algebra. Thus the grading is toral. Finally, for $j=110$ we have $A(110$, id $)=\left\langle t_{-1,-1,-1,1}^{\prime}, t_{1,1,1,-1}^{\prime}, \widetilde{\sigma_{110}}\right\rangle$, which produces a $\mathbb{Z}_{2}^{2} \times \mathbb{Z}_{4}$-grading. In this case the zero homogeneous component is a six-dimensional (reductive) algebra $L_{e}=\left\langle y_{1}, \ldots, y_{6}\right\rangle$ where

$$
\begin{array}{ll}
y_{1}=b_{3}-b_{10}+b_{27}+b_{34}, & y_{4}=-b_{4}+b_{22}+b_{28}+b_{46}, \\
y_{2}=-b_{7}-b_{18}-b_{31}+b_{42}, & y_{5}=b_{13}-b_{23}-b_{37}+b_{47}, \\
y_{3}=b_{16}-b_{20}-b_{40}+b_{44}, & y_{6}=b_{15}-b_{24}-b_{39}+b_{48},
\end{array}
$$

which has rank 4 because $\left\{y_{1}-y_{6}, y_{2}-y_{5}, y_{3}-y_{4}\right\}$ is contained in the center of $L_{e}$ (there are only two types of six-dimensional reductive subalgebras, $\mathfrak{a}_{1}$ plus a three-dimensional center and $2 \mathfrak{a}_{1}$, of ranks 4 and 2 respectively). Hence the grading is toral.

Remark 1. Notice that the order of $\sigma_{i}$ does not necessarily coincide with the order of $\widetilde{\sigma}_{i}$, but it is a divisor. This happens, for instance, for $i=3$, since $\sigma_{3}$ has order 4 while $\widetilde{\sigma}_{3}$ has order 8 . This is not because of a bad choice of $\widetilde{\sigma}_{3}$, since all the possible extensions of $\sigma_{3}$ have the same order, as the next lemma shows. 
Lemma 1. Take $j \in\{1, \ldots, 1152\}$, and $m$ the order of $\sigma_{j} \in \mathcal{W}$. Then the following conditions are equivalent:

i) $\mathfrak{T}^{\langle j\rangle}$ is finite,

ii) $\mathfrak{T}^{\langle j\rangle} \subset\left\{t_{x, y, z, u}^{\prime} \mid x^{m}=y^{m}=z^{m}=u^{m}=1\right\}$,

iii) $\left(\widetilde{\sigma}_{j} t\right)^{m}=\widetilde{\sigma}_{j}^{m}$ for any $t \in \mathfrak{T}$,

iv) All the elements in $\left\{f \in \mathfrak{N} \mid \pi(f)=\sigma_{j}\right\}$ (where $\pi: \mathfrak{N} \rightarrow \mathcal{W}$ is the canonical projection) have the same order.

Proof. Take the element in $\mathfrak{T}$ given by

$$
s_{x, y, z, u}:=t_{x, y, z, u}^{\prime}\left(\widetilde{\sigma}_{j} t_{x, y, z, u}^{\prime} \widetilde{\sigma}_{j}^{-1}\right)\left(\widetilde{\sigma}_{j}^{2} t_{x, y, z, u}^{\prime} \widetilde{\sigma}_{j}^{-2}\right) \ldots\left(\widetilde{\sigma}_{j}^{m-1} t_{x, y, z, u}^{\prime} \widetilde{\sigma}_{j}^{1-m}\right)
$$

(product of elements in $\mathfrak{T}$ ). Since $\sigma_{j}^{m}=\mathrm{id}$, we have $\widetilde{\sigma}_{j}^{m} \in \mathfrak{T}$ and thus $\tilde{\sigma}_{j} s_{x, y, z, u} \widetilde{\sigma}_{j}^{-1}=s_{x, y, z, u}$, that is, $s_{x, y, z, u} \in \mathfrak{T}^{\langle j\rangle}$. Besides it verifies that

$$
\left(\widetilde{\sigma}_{j} t_{x, y, z, u}^{\prime}\right)^{m}=s_{x, y, z, u} \widetilde{\sigma}_{j}^{m} .
$$

The implication $i i) \Rightarrow i$ ) is trivial. Now, if we assume $i i i), s_{x, y, z, u}=\mathrm{id}$ for any $x, y, z, u \in F^{\times}$. But if $t_{x, y, z, u}^{\prime} \in \mathfrak{T}^{\langle j\rangle}$, then $\left(t_{x, y, z, u}^{\prime}\right)^{m}=s_{x, y, z, u}$, and so $t^{m}=$ id for any $t \in \mathfrak{T}^{\langle j\rangle}$, and we have $\left.i i\right)$.

Next suppose $i$ ). We have $s_{x, y, z, u}=t_{f_{1}(x, y, z, u), f_{2}(x, y, z, u), f_{3}(x, y, z, u), f_{4}(x, y, z, u)}^{\prime}$ for some rational functions $f_{i} \in F(x, y, z, u)$. But the set $\left\{s_{x, y, z, u} \mid x, y, z, u \in\right.$ $\left.F^{\times}\right\} \subset \mathfrak{T}^{\langle j\rangle}$ must be finite, so the fractions are constant $s_{x, y, z, u}=t_{a, b, c, d}^{\prime}$, and, since $s_{1,1,1,1}=\mathrm{id}$, the identity $s_{x, y, z, u}=\mathrm{id}$ holds for any $x, y, z, u \in F^{\times}$. This gives iii).

From $i i i)$, we conclude that the orders of $\widetilde{\sigma}_{j} t$ and $\widetilde{\sigma}_{j}$ are the same, since none of them can be less than $m$ and $\left(\widetilde{\sigma}_{j} t\right)^{m}=\widetilde{\sigma}_{j}^{m}$, so we have $i v$ ).

Conversely, let $c$ be such that $m c$ is the order of $\widetilde{\sigma}_{j} t_{x, y, z, u}^{\prime}$ for any $x, y, z, u$. Thus id $=\left(\widetilde{\sigma}_{j} t_{x, y, z, u}^{\prime}\right)^{m c}=\left(s_{x, y, z, u} \widetilde{\sigma}_{j}^{m}\right)^{c}=s_{x, y, z, u}^{c}$, hence $f_{i}(x, y, z, u)^{c}=1$ and so $s_{x, y, z, u}=\mathrm{id}$, which is equivalent to $\left.i i i\right)$.

Remark 2. Apparently the grading induced by the quasitorus $A(3, \mathrm{id})=$ $\left\langle\left\{\widetilde{\sigma_{3}}, t_{1,-1,-1, i}^{\prime}, t_{-1,-1,1,1}^{\prime}\right\}\right\rangle$ is a $\mathbb{Z}_{8} \times \mathbb{Z}_{4} \times \mathbb{Z}_{2}$-grading, since 8,4 and 2 are respectively the orders of the generators. However, the right group generated by the support is $\mathbb{Z}_{2}^{2} \times \mathbb{Z}_{8}$ because $\left(\widetilde{\sigma}_{3}\right)^{2} t_{1,-1,-1, i}^{\prime}$ has order 2 .

Returning to our quasitori $A(j, t)$, the toral element $t$ plays a secondary role.

Proposition 6. If $j \in\{3,15,105,106,405\}$, the quasitorus $A(j, t)$ is conjugated to $A\left(j, t^{\prime}\right)$ for any $t, t^{\prime} \in \mathfrak{T}$. 
Proof. Take

$$
\mathfrak{S}^{\langle j\rangle}=\left\{\widetilde{\sigma}_{j}^{-1} t \widetilde{\sigma}_{j} t^{-1} \mid t \in \mathfrak{T}\right\} .
$$

Denoting $\widetilde{\sigma}_{j}$ by $\sigma$, we have that $\left(\sigma^{-1} t \sigma t^{-1}\right)\left(\sigma^{-1} s \sigma s^{-1}\right)=\sigma^{-1} t s \sigma t^{-1} \sigma^{-1} \sigma s^{-1}=$ $\sigma^{-1} t s \sigma(t s)^{-1}$, since $\sigma t^{-1} \sigma^{-1} \in \mathfrak{T}$ and so it commutes with $s$. Thus $\mathfrak{S}^{\langle j\rangle}$ is a subgroup. Besides it has the property that $A(j, \mathrm{id})$ is conjugated to $A(j, s)$ for any $s \in \mathfrak{S}^{\langle j\rangle}$. Indeed, if $s=\sigma^{-1} t \sigma t^{-1}$, then $\operatorname{Ad}(t)\left(\mathfrak{T}^{\langle j\rangle}\right)=\mathfrak{T}^{\langle j\rangle}$ and $\operatorname{Ad}(t)(\sigma)=\sigma s$.

On the other hand, it is clear that $A(j, s)=A(j, s t)$ for any $t \in \mathfrak{T}^{\langle j\rangle}$, therefore

$$
A(j, \mathrm{id}) \cong A(j, s t)
$$

for any $s \in \mathfrak{S}^{\langle j\rangle}, t \in \mathfrak{T}^{\langle j\rangle}$. Let us see that $\mathfrak{T}^{\langle j\rangle} \mathfrak{S}^{\langle j\rangle}=\mathfrak{T}$. First we observe that the map

$$
\begin{array}{ll}
\mathfrak{T} / \mathfrak{T}^{\langle j\rangle} & \rightarrow \mathfrak{S}^{\langle j\rangle} \\
{[t]} & \mapsto \widetilde{\sigma}_{j}^{-1} t \widetilde{\sigma}_{j} t^{-1}
\end{array}
$$

is a group isomorphism. It is well defined and injective because $t \in \mathfrak{T}^{\langle j\rangle}$ if and only if $\widetilde{\sigma}_{j}^{-1} t \widetilde{\sigma}_{j} t^{-1}=\mathrm{id}$. In particular $\operatorname{dim} \mathfrak{T}=\operatorname{dim} \mathfrak{T}^{\langle j\rangle}+\operatorname{dim} \mathfrak{S}^{\langle j\rangle}($ see $[31$, Proposition 2.26, p.41]). And we have another isomorphism:

$$
\mathfrak{S}^{\langle j\rangle} / \mathfrak{S}^{\langle j\rangle} \cap \mathfrak{T}^{\langle j\rangle} \rightarrow \mathfrak{S}^{\langle j\rangle} \mathfrak{T}^{\langle j\rangle} / \mathfrak{T}^{\langle j\rangle},
$$

hence $\operatorname{dim} \mathfrak{T}^{\langle j\rangle}+\operatorname{dim} \mathfrak{S}^{\langle j\rangle}=\operatorname{dim} \mathfrak{S}^{\langle j\rangle} \mathfrak{T}^{\langle j\rangle}+\operatorname{dim} \mathfrak{S}^{\langle j\rangle} \cap \mathfrak{T}^{\langle j\rangle}$.

But $\mathfrak{S}^{\langle j\rangle} \cap \mathfrak{T}^{\langle j\rangle}$ is a finite group for any of our indices $j$. Indeed, for $j=3,15,106,405$ the group $\mathfrak{T}^{\langle j\rangle}$ is already finite, and for $j=105$,

$\mathfrak{S}^{\langle 105\rangle}=\left\{t_{x, y, z, u}^{\prime} \mid u^{2}=x y z\right\}, \quad \mathfrak{T}^{\langle 105\rangle}=\left\{t_{x, y, z, u}^{\prime} \mid x^{2}=y^{2}=1, z=x y, u \in F^{\times}\right\}$

and

$$
\mathfrak{S}^{\langle 105\rangle} \cap \mathfrak{T}^{\langle 105\rangle}=\left\{t_{x, y, x y, u x y}^{\prime} \mid x^{2}=y^{2}=u^{2}=1\right\} \cong \mathbb{Z}_{2}^{3} .
$$

Consequently $\operatorname{dim} \mathfrak{T}=\operatorname{dim} \mathfrak{T}^{\langle j\rangle}+\operatorname{dim} \mathfrak{S}^{\langle j\rangle}=\operatorname{dim} \mathfrak{S}^{\langle j\rangle} \mathfrak{T}^{\langle j\rangle}$ and so $\mathfrak{T}^{\langle j\rangle} \mathfrak{S}^{\langle j\rangle}=\mathfrak{T}$.

Furthermore, $\mathfrak{S}^{\langle j\rangle} \cap \mathfrak{T}^{\langle j\rangle}$ is a finite group for all $j \in I$, so also for any $j \in\{1, \ldots 1152\}$, and thus $A(j, t) \cong A\left(j, t^{\prime}\right)$ for all $t, t^{\prime} \in \mathfrak{T}$, although this fact is unnecessary for our purposes.

As a consequence of this lemma, if $\mathfrak{T}^{\langle j\rangle}$ is finite, then $\mathfrak{S}^{\langle j\rangle}=\mathfrak{T}$, and every $t \in \mathfrak{T}$ is in $\mathfrak{S}^{\langle j\rangle}$, that is, there is $s \in \mathfrak{T}$ such that $t=\widetilde{\sigma}_{j}^{-1} s^{-1} \widetilde{\sigma}_{j} s$. Thus we have obtained the following technical result, which will be very useful in some forthcoming proofs.

Corollary 2. If $\mathfrak{T}^{\langle j\rangle}$ is finite, for any $t \in \mathfrak{T}$ there is $s \in \mathfrak{T}$ such that

$$
s \widetilde{\sigma}_{j} t s^{-1}=\widetilde{\sigma}_{j} .
$$


The relevance of the quasitori $A(j, t)$ is highlighted by the following result.

Proposition 7. Let $F=\left\{f_{1}, \ldots, f_{n}, f_{n+1}\right\}$ be a nontoral commutative family of semisimple elements in a connected algebraic group $G$ such that the subset $\left\{f_{1}, \ldots, f_{n}\right\}$ is toral. Fix a maximal torus $T$ of $G$. Then, the subgroup generated by $F$ is conjugated to some subgroup of the form $\left\langle t_{1}, \ldots, t_{n}, \sigma\right\rangle$ where $t_{i} \in T$ and $\sigma \in \mathfrak{N}_{G}(T)$ is conjugated to $f_{n+1}$.

Proof. Define $Z$ as the centralizer of $\left\{f_{1}, \ldots, f_{n}\right\}$ in $G$ and let $T^{\prime}$ be some maximal torus in $G$ containing $\left\{f_{1}, \ldots, f_{n}\right\}$. The subgroup $\overline{\langle F\rangle} \subset Z$ is a quasitorus of $Z$, hence is contained in the normalizer $\mathfrak{N}_{Z}\left(T^{\prime \prime}\right)$ of some maximal torus $T^{\prime \prime}$ in $Z$. Then $T^{\prime \prime}$ is also a maximal torus in $G$ (since $T^{\prime} \subset Z$ ) and there is some $p \in G$ such that $T^{\prime \prime}=p T p^{-1}$. On the other hand $\left\{f_{1}, \ldots, f_{n}\right\}$ is contained in the center of $Z$, and since these are semisimple elements, then they are contained in each maximal torus of $Z$. In particular $f_{i} \in T^{\prime \prime}$ for $i \in\{1, \ldots, n\}$, and $f_{n+1} \in \mathfrak{N}_{Z}\left(T^{\prime \prime}\right) \subset \mathfrak{N}_{G}\left(T^{\prime \prime}\right)$. Thus, $p^{-1}\langle F\rangle p$ is generated by $f_{i}^{\prime}=p^{-1} f_{i} p$ for $i=1, \ldots, n+1$, with $f_{i}^{\prime} \in T$ for $i \leq n$ and $f_{n+1}^{\prime} \in p^{-1} \mathfrak{N}_{G}\left(T^{\prime \prime}\right) p=\mathfrak{N}_{G}(T)$.

In this way we have proved that any nontoral grading has a coarsening isomorphic to a grading induced by a subquasitorus of $A(j, t)$ for some $j \in$ $\{3,15,105,106,405\}$. Furthermore, by Proposition 6 , the element $t$ can be taken to be the identity. We can even remove two more possibilities for $j$, as the following corollary shows.

Corollary 3. Each of the subgroups $A(3, i d)$ and $A(106, i d)$ of $\mathfrak{F}_{4}$ is conjugated to a subgroup of $A(105, i d)$.

Proof. We know that $A(3, \mathrm{id})=\left\langle\left\{t_{-1,-1,1,1}^{\prime}, t_{1,-1,-1, i}^{\prime}, \widetilde{\sigma}_{3}\right\}\right\rangle$ so that making $f_{1}=t_{1,-1,-1, i}^{\prime}, f_{2}=\widetilde{\sigma}_{3}$ and $f_{3}=t_{-1,-1,1,1}^{\prime}$ we can apply the previous proposition to $F=\left\{f_{1}, f_{2}, f_{3}\right\}$. Of course $\left\{f_{1}, f_{2}\right\}$ is toral by Lemma 2, while $A(3, \mathrm{id})$ is nontoral as proved in Proposition 5. Thus $A(3, \mathrm{id})$ is conjugated to a group of the form $\left\langle t_{1}, t_{2}, \sigma\right\rangle$ with $t_{1}, t_{2} \in \mathfrak{T}$ and $\sigma \in \mathfrak{N}$. Moreover $\sigma$ has order 2 since it is conjugated to $t_{-1,-1,1,1}^{\prime}$. We also know that $\sigma=\widetilde{\sigma}_{i} t$ for some $t \in \mathfrak{T}$ and $i \in\{1, \ldots, 1152\}$. Since $\sigma$ has order two, the same can be said about $\sigma_{i}$. That is, $A(3, \mathrm{id})$ is conjugated to some subgroup of $A(i, t)$ with $\sigma_{i}$ of order two. Furthermore $A(i, t)$ is nontoral so that, applying Proposition 4 , the quasitorus $A(i, \mathrm{id})$ is nontoral. We can choose $i \in I$ by conjugating if necessary. Now Proposition 5 implies that $i=3,15,105,106$ or 405. We get, by Proposition 6 , that the quasitorus $A(3$, id) is conjugated to some subgroup of $A(i, \mathrm{id})$. Since $\sigma_{i}^{2}=1$ the only possible values for $i$ are 105 and 405 . If we had a copy of $A(3, \mathrm{id})$ within $A(405$,id) then this group (isomorphic to $\mathbb{Z}_{2}^{5}$ ) should contain an element of order 8 . 
Consider now $A(106, \mathrm{id})=\left\langle\left\{t_{-1,1,-1,1}^{\prime}, t_{1,-1,-1,-1}^{\prime}, \widetilde{\sigma_{106}}\right\}\right\rangle$ and apply the previous proposition to $F=\left\{f_{1}, f_{2}, f_{3}\right\}$ with $f_{1}=\widetilde{\sigma_{106}}, f_{2}=t_{-1,1,-1,1}^{\prime}$ and $f_{3}=t_{1,-1,-1,-1}^{\prime}$. As before $A(106, \mathrm{id})$ is conjugated to a subgroup of a nontoral $A\left(i\right.$,id) with $\sigma_{i}$ of order two. Again we can take $i \in I$ so that $i=105$ or 405 , but $A(405$, id $)$ contains no order six element.

Our objective in the next subsection is to show that in fact any nontoral grading is isomorphic to one produced by a quasitorus contained in $A(j, \mathrm{id})$ for $j=15,105,405$.

\subsection{Fine gradings}

Next we study the maximality of some of the previous quasitori. As a first step, their maximality in $\mathfrak{N}$, the normalizer of the maximal torus $\mathfrak{T}$, is clear:

Proposition 8. Let $A=A(j, i d)$ for $j \in\{15,105,405\}$. Then $A$ is its own centralizer in $\mathfrak{N}$, that is, $\mathfrak{C}_{\mathfrak{N}}(A)=A$.

Proof. To prove $\mathfrak{C}_{\mathfrak{N}}(A) \subset A$ take $f \in \mathfrak{C}_{\mathfrak{N}}(A)$. Since $f \in \mathfrak{N}$ there is some $i \in\{1, \ldots, 1152\}$ and some $t \in \mathfrak{T}$ such that $f=\widetilde{\sigma}_{i} t$. Consider first the possibility $j=405$. Of course $f$ commutes with each element in $\mathfrak{T}^{\langle 405\rangle} \subset A$ implying that $\widetilde{\sigma}_{i}$ does the same. Consequently $\mathfrak{T}^{\langle 405\rangle} \subset \mathfrak{T}^{\langle i\rangle}$. According to Table (4.10), this is possible only for $i=405$ or $i=748$ (take into account that any $\mathfrak{T}^{\langle i\rangle}$ is isomorphic to some in the table, that the unique groups in the table which may contain a copy of $\mathbb{Z}_{2}^{4}$ are $\mathfrak{T}^{\langle 405\rangle}$ and $\mathfrak{T}^{\langle 748\rangle}$, and that the orbits of $\sigma_{405}$ and $\sigma_{748}$ have cardinal one). So $\widetilde{\sigma}_{i}$ (equal to $\widetilde{\sigma_{405}}$ or $\left.\widetilde{\sigma_{748}}\right)$ commutes with $\widetilde{\sigma_{405}}$, and since $f=\widetilde{\sigma}_{i} t$ also does, this forces the commutativity of $t$ and $\widetilde{\sigma_{405}}$ so that $t \in \mathfrak{T}^{\langle 405\rangle}$ and $f=\widetilde{\sigma}_{i} t \in A(405$, id). Let us consider now the case $j=15$. From $f=\widetilde{\sigma}_{i} t$ and the fact that $f$ commutes with $\mathfrak{T}^{\langle 15\rangle}$ we get that $\widetilde{\sigma}_{i}$ commutes with $\mathfrak{T}^{\langle 15\rangle}$. Therefore $\mathfrak{T}^{\langle 15\rangle} \subset \mathfrak{T}^{\langle i\rangle}$ and as $\mathfrak{T}^{\langle 15\rangle}=\left\langle t_{\omega, 1, \omega^{2}, \omega^{2}}^{\prime}, t_{1, \omega, \omega, 1}^{\prime}\right\rangle$, we conclude $\sigma_{i} \cdot t=t$ for both $t=t_{\omega, 1, \omega^{2}, \omega^{2}}^{\prime}$ and $t=t_{1, \omega, \omega, 1}^{\prime}$. But using (4.9) we see that the unique values of $i$ satisfying the above relations are 15,748 and 1075. Hence either $\sigma_{i}=\sigma_{15}$ or $\sigma_{i}=\sigma_{1075}=$ $\sigma_{15}^{2}$ or $\sigma_{i}=\sigma_{748}=\sigma_{15}^{3}$. In any case $\widetilde{\sigma}_{i}$ commutes with $\widetilde{\sigma_{15}}$ (which is not automatic from the commutativity of $\sigma_{i}$ and $\sigma_{15}$, but can be proved directly for those indices or checking the equalities $\widetilde{\sigma_{15}^{n}}={\widetilde{\sigma_{15}}}^{n}$ for $\left.n=2,3\right)$. Thus, recalling that $f$ commutes with $\widetilde{\sigma_{15}}$ we get that $t$ commutes with $\widetilde{\sigma_{15}}$, that is, $t \in \mathfrak{T}^{\langle 15\rangle} \subset A\left(15\right.$,id). Therefore $f=\widetilde{\sigma}_{i} t \in A(15$,id). Finally, we must investigate the case $j=105$. Since $f=\widetilde{\sigma}_{i} t$ commutes with $A(105$, id $)$ and $\mathfrak{T}^{\langle 105\rangle}$ is generated by $t_{-1,1,-1,1}^{\prime}, t_{1,-1,-1,1}^{\prime}$ and $t_{1,1,1, u}^{\prime}$ with $u \in F^{\times}$, we must have that $\widetilde{\sigma}_{i}$ commutes with any of the mentioned generators. So solving in $i$ the system $\sigma_{i} \cdot t=t$ for $t \in\left\{t_{-1,1,-1,1}, t_{1,-1,-1,1}, t_{1,1,1, u} \mid u \in F^{\times}\right\}$, we get that $i \in\{105,748\}$. But $\widetilde{\sigma_{105}}$ and $\widetilde{\sigma_{748}}$ commute and consequently $\widetilde{\sigma_{i}}$ commutes with $\widetilde{\sigma_{105}}$. As before we get that $t \in \mathfrak{T}^{\langle 105\rangle}$, implying $f \in A(105$, id). 
We must emphasize that if the centralizer (in $\mathfrak{N}$ ) of a subgroup agrees with the subgroup itself, this does not imply that the centralizer of the group in $\mathfrak{F}_{4}$ agrees with the group. However this will be the case for some special relevant subgroups in our work. For the next result we must recall from 2.1 that if we have a maximal abelian subgroup of semisimple elements in aut $(L)$ for a Lie algebra $L$, then the induced grading on $L$ is fine.

Proposition 9. The gradings induced by $A(j, i d)$ for $j=15,105,405$ are fine.

Proof. Let $A=A(j$,id $)$ and suppose that the induced grading is not fine. Then there is some semisimple $f \in \mathfrak{C}_{\mathfrak{F}_{4}}(A) \backslash A$ such the grading induced by $A \cup\{f\}$ is a proper refinement of the original grading (proper in the sense that it is different from the given grading). Define $Z=\mathfrak{C}_{\mathfrak{F}_{4}}\left(\mathfrak{T}^{\langle j\rangle}\right)$. Then the group $\langle A \cup\{f\}\rangle$ is an abelian subgroup of $Z$ and so is its closure $\overline{\langle A \cup\{f\}\rangle}$ in the Zarisky topology. But this is again a quasitorus, whence it is contained in the normalizer of some maximal torus $T$ of $Z$. In particular $\langle A \cup\{f\}\rangle \subset \mathfrak{N}_{Z}(T)$ and by construction also $\mathfrak{T} \subset Z$ so that there is some $p \in Z$ such that $p T p^{-1}=\mathfrak{T}$. Consequently $p \mathfrak{N}_{\mathfrak{F}_{4}}(T) p^{-1}=\mathfrak{N}_{\mathfrak{F}_{4}}(\mathfrak{T})=\mathfrak{N}$. Thus $p\langle A \cup\{f\}\rangle p^{-1} \subset \mathfrak{N}$ and

$$
p f p^{-1}, p \widetilde{\sigma}_{j} p^{-1} \in \mathfrak{N} \cap \mathfrak{C}_{\mathfrak{F}_{4}}\left(\mathfrak{T}^{\langle j\rangle}\right)
$$

with $p t p^{-1}=t$ for any $t \in \mathfrak{T}^{\langle j\rangle}$.

For $j=105$ we have $\mathfrak{N} \cap \mathfrak{C}_{\mathfrak{F}_{4}}\left(\mathfrak{T}^{\langle 105\rangle}\right)=\mathfrak{T} \cup \widetilde{\sigma_{105}} \mathfrak{T}$, taking into account the previous proposition. Now we must analyze different possibilities:

- If $p \widetilde{\sigma_{105}} p^{-1} \in \mathfrak{T}$, then $p A p^{-1} \subset \mathfrak{T}$ and the grading induced by $A$ would be toral, which is a contradiction.

- If $p f p^{-1} \in \mathfrak{T}$ and $p \widetilde{\sigma_{105}} p^{-1}=\widetilde{\sigma_{105}} t$ for some $t \in \mathfrak{T}$, it is clear that $p f p^{-1} \in \mathfrak{T}^{\langle 105\rangle}$ (it commutes with $\widetilde{\sigma_{105}} t$ ) and $p A p^{-1} \subset A(105, t)$. Hence $\langle A \cup\{f\}\rangle \subset p^{-1} A(105, t) p$ and it cannot produce a proper refinement. This is another contradiction.

- Thus we have $p f p^{-1}=\widetilde{\sigma_{105}} t_{1}$ and $\widetilde{p \sigma_{105}} p^{-1}=\widetilde{\sigma_{105}} t_{2}$ for some $t_{1}, t_{2} \in \mathfrak{T}$. Since both elements commute we get easily that $t_{1} t_{2}^{-1} \in \mathfrak{T}^{\langle 105\rangle}$. Therefore $p f p^{-1} \in p \widetilde{\sigma_{105}} p^{-1} \mathfrak{T}^{\langle 105\rangle}=p \widetilde{\sigma_{105}} \mathfrak{T}^{\langle 105\rangle} p^{-1} \subset p A p^{-1}$ implying $f \in A$, a contradiction again.

The case $j=405$ is proved analogously since $\mathfrak{N} \cap \mathfrak{C}_{\mathfrak{F}_{4}}\left(\mathfrak{T}^{\langle 405\rangle}\right)=\mathfrak{T} \cup \widetilde{\sigma_{405}} \mathfrak{T}$. However for $j=15$ we need some slight modifications since $\mathfrak{N} \cap \mathfrak{C}_{\mathfrak{F}_{4}}\left(\mathfrak{T}^{\langle 15\rangle}\right)=$ $\mathfrak{T} \cup \widetilde{\sigma_{15}} \mathfrak{T} \cup\left(\widetilde{\sigma_{15}}\right)^{2} \mathfrak{T}$. Let us use the notation $\sigma:=\widetilde{\sigma_{15}}$ for simplicity. Since $p f p^{-1}, p \sigma p^{-1} \in \mathfrak{N} \cap \mathfrak{C}_{\mathfrak{F}_{4}}\left(\mathfrak{T}^{\langle 15\rangle}\right)$, we have several possibilities. 
- If $p f p^{-1}$ or $p \sigma p^{-1}$ is of the form $\sigma t$ for some $t \in \mathfrak{T}$, applying Corollary 2, there is some $s \in \mathfrak{T}$ such that $s \sigma t s^{-1}=\sigma$. This implies that $\mathfrak{T}^{\langle 15\rangle} \cup\{\sigma\} \subset \operatorname{sp}\langle A \cup\{f\}\rangle(s p)^{-1} \subset \mathfrak{N}$ and by Proposition 8 we have $\mathfrak{C}_{\mathfrak{N}}(A(15, \mathrm{id}))=A(15, \mathrm{id})$ implying $s p\langle A \cup\{f\}\rangle(s p)^{-1}=A(15, \mathrm{id})=A$. Thus the grading induced by $s p\langle A \cup\{f\}\rangle(s p)^{-1}$, which is equivalent to a proper refinement of the one induced by $A$, is also equivalent to $A$, a contradiction.

- If $p f p^{-1}$ or $p \sigma p^{-1}$ is of the form $\sigma^{2} t$ for some $t \in \mathfrak{T}$, applying again Corollary 2, there is some $s \in \mathfrak{T}$ such that $s \sigma^{2} t s^{-1}=\sigma^{2}=\sigma_{1075}$. This implies that $\mathfrak{T}^{\langle 15\rangle} \cup\left\{\sigma^{2}\right\} \subset s p\langle A \cup\{f\}\rangle(s p)^{-1} \subset \mathfrak{N}$ and since $\mathfrak{T}^{\langle 15\rangle} \cup\left\{\sigma^{2}\right\}$ generates $A(15, \mathrm{id})=A(1075$, id $)$, we conclude as before.

- And if $p f p^{-1}$ and $p \sigma p^{-1}$ are both in $\mathfrak{T}$, the grading is obviously toral.

We finish this section with one of our main results. Before proceeding with its precise statement and proof, we must realize that any quasitorus $A$ in an algebraic group $G$ has a (not necessarily unique) maximal toral part, a toral subgroup $B$ which is not contained in another toral subgroup of $A$. This is trivial if the quasitorus $A$ has finite cardinal. Otherwise consider the family $\mathcal{F}$ of all the toral subgroups of $A$. The elements in $\mathcal{F}$ are toral in the sense that each of them is contained in some torus of $G$. To see that $\mathcal{F}$ has maximal elements we consider a maximal subtorus $T$ of $A$. For any $B \in \mathcal{F}$ containing $T$ (for instance, $T$ verifies this) we have $T \subset B_{0}$ (the unit component of $B$ ) and $B_{0} \subset A_{0}=T$. Thus $B_{0}=T$ and the quotient group $B / T$ is finite and its cardinal is bounded by that of $A / T$. Hence any $B \in \mathcal{F}$ with $T \subset B$ and such that $B / T$ has maximal cardinal can be proved to be a maximal element in $\mathcal{F}$. Such subgroups are what we shall understand as maximal toral subgroups of the quasitorus $A$.

Theorem 4. Let $A \subset \mathfrak{F}_{4}$ be a quasitorus, then up to conjugacy, A falls in one of the following cases:

- $A \subset \mathfrak{T}$ (maximal torus).

- $A \subset A(105, i d)$.

- $A \subset A(405, i d)$.

- $A=A(15, i d)$.

Proof. The precise meaning of this theorem is that there is some $\phi \in \mathfrak{F}_{4}$ such that $A^{\prime}:=\phi A \phi^{-1}$ is in some of the cases above. So we can replace at any moment $A$ with some of its conjugated subgroups in the group $\mathfrak{F}_{4}$. From the beginning we suppose that $A$ is nontoral. Consider a maximal toral subgroup $B$ of $A$ which may be supposed to be a subgroup of the maximal torus $\mathfrak{T}$ defined in Subsection 4.2. Define now the group $Z:=\mathfrak{C}_{\mathfrak{F}_{4}}(B)$ which 
contains both $A$ and $\mathfrak{T}$. Since $A$ is a quasitorus of $Z$ there is a maximal torus $T \subset Z$ such that $A \subset \mathfrak{N}_{Z}(T)$ (see the first paragraph in Section 5). But $\mathfrak{T}, T \subset Z$ are maximal tori in $Z$ so that there exists $p \in Z$ such that $\mathfrak{T}=p T p^{-1}$. Thus $p A p^{-1} \subset \mathfrak{N}_{Z}(\mathfrak{T}) \subset \mathfrak{N}_{\mathfrak{F}_{4}}(\mathfrak{T})=\mathfrak{N}$ and since $p$ centralizes $B$, the subgroup $B=p B p^{-1}$ is still a toral maximal subgroup of $p A p^{-1}$. In this way, replacing $A$ by $p A p^{-1}$ we can suppose that $A \subset \mathfrak{N}$ with $B \subset \mathfrak{T}$, $B$ a maximal toral subgroup of $A$. Furthermore $A=\left\langle B \cup\left\{f_{1}, \ldots f_{k}\right\}\right\rangle$ with $f_{i} \in \mathfrak{N}$ so that each $f_{i}$ is of the form $\widetilde{\sigma}_{j} t$ with $j \in\{1, \ldots, 1152\}$ and $t \in \mathfrak{T}$. Moreover, for any $\widetilde{\sigma}_{j} t \in A$ we have $B \subset \mathfrak{T}^{\langle j\rangle}$ since any element in $B$ centralizes $A$ and $\mathfrak{T}$ and so it commutes with $\widetilde{\sigma}_{j}$. On the other hand, $\left\langle B \cup\left\{\widetilde{\sigma}_{j} t\right\}\right\rangle$ being nontoral and contained in $A(j, t)$ implies that $\sigma_{j}$ is in the orbit (under conjugation) of $\sigma_{3}, \sigma_{15}, \sigma_{105}, \sigma_{106}$ or $\sigma_{405}$ (see Proposition 5). Next we analyze the different possibilities arising.

- If some of the $\sigma_{j}$ 's is in the orbit of $\sigma_{15}$ we can suppose that $f_{1}=\widetilde{\sigma}_{j} t$. Then $f_{1}$ is conjugated in $\mathfrak{N}$ to $\widetilde{\sigma_{15}} t^{\prime}$ for some $t^{\prime} \in \mathfrak{T}$ and without loss in generality we can take $f_{1}=\widetilde{\sigma_{15}}$, by Corollary 2 (the element $s \in \mathfrak{T}$ does not change neither $\mathfrak{T}$ nor $B$ ). Thus $B \subset \mathfrak{T}^{\langle 15\rangle} \cong \mathbb{Z}_{3}^{2}$. Since $\left\langle B \cup\left\{f_{1}\right\}\right\rangle$ is nontoral, then it has at least three generators (see Lemma 2 in the Appendix). Consequently $B$ has at least two generators, and this implies $B=\mathfrak{T}^{\langle 15\rangle}$. So $A(15$,id $) \subset A \subset \mathfrak{N}$ and applying Proposition 8, we have $\mathfrak{C}_{\mathfrak{N}}(A(15, \mathrm{id}))=A(15$, id $)$, hence $A=A(15$, id $)$.

- Some of the $\sigma_{j}$ 's is conjugated to $\sigma_{106}$. As before $f_{1}$ can be taken to be $f_{1}=\widetilde{\sigma_{106}}$ and $B \subset \mathfrak{T}^{\langle 106\rangle} \cong \mathbb{Z}_{2}^{2}$. Since $B$ must have at least two generators we have $B=\mathfrak{T}^{\langle 106\rangle}$. Now, for any other $f_{i}=\widetilde{\sigma}_{k} t$ we must have $\mathfrak{T}^{\langle 106\rangle} \subset \mathfrak{T}^{\langle k\rangle}$. The commutativity of $f_{1}$ and $f_{i}$ implies that of $\sigma_{106}$ and $\sigma_{k}$. But a computation of the number of $k$ 's satisfying both conditions

$$
\left\{\begin{array}{l}
\mathfrak{T}^{\langle 106\rangle} \subset \mathfrak{T}^{\langle k\rangle}, \\
\sigma_{106} \sigma_{k}=\sigma_{k} \sigma_{106},
\end{array}\right.
$$

reveals that there are only six possible values of $k$. Since $\sigma_{106}$ has order 6 , obviously the six powers $\sigma_{106}^{n}$ with $n \in\{0, \ldots, 5\}$ satisfy the conditions so that there is $n \in \mathbb{N}$ and $t_{1} \in \mathfrak{T}$ with $f_{i}={\widetilde{\sigma_{106}}}^{n} t_{1}$. But $t_{1} \in \mathfrak{T}^{\langle 106\rangle}$ again by the commutativity of $f_{i}$ with $f_{1}$, so $f_{i} \in A(106$,id). From here $A=A(106$, id) which up to conjugacy is a subgroup of $A(105$, id $)$ by Corollary 3.

- Suppose now that some of the $\sigma_{j}$ 's is in the orbit of $\sigma_{3}$. So we can suppose $f_{1}=\sigma_{3}$ without loss of generality, and $B \subset \mathfrak{T}^{\langle 3\rangle}=$ $\left\langle\left\{t_{-1,-1,1,1}^{\prime}, t_{-1,1,-1, i}^{\prime}\right\}\right\rangle$, which is isomorphic to $\mathbb{Z}_{2} \times \mathbb{Z}_{4}$. But again by Lemma 2 , the subgroup $B$ must have at least two generators since $\langle B \cup$ $\left.\left\{f_{1}\right\}\right\rangle$ is nontoral. Whence either $B=\mathfrak{T}^{\langle 3\rangle}$ or $B=\left\langle\left\{t_{-1,-1,1,1}^{\prime}, t_{1,1,1,-1}^{\prime}\right\}\right\rangle$. 
But this last possibility can not occur because then $\left\langle B \cup\left\{f_{1}\right\}\right\rangle=$ $\left\langle\left\{t_{-1,-1,1,1}^{\prime}, t_{1,1,1,-1}^{\prime}, \widetilde{\sigma_{3}}\right\}\right\rangle$ would be toral because ${\widetilde{\sigma_{3}}}^{4}=t_{1,1,1,-1}$, hence $\left\langle B \cup\left\{f_{1}\right\}\right\rangle$ would have two generators. So necessarily $B=\mathfrak{T}^{\langle 3\rangle}$ and for any other $f_{i}=\widetilde{\sigma}_{k} t$ we must have $\mathfrak{T}^{\langle 3\rangle} \subset \mathfrak{T}^{\langle k\rangle}$ and $\sigma_{3} \sigma_{k}=\sigma_{k} \sigma_{3}$. We obtain only four possible $k$ 's satisfying the previous conditions. Hence $\sigma_{k} \in\left\{\sigma_{3}^{n} \mid n=0,1,2,3\right\}$ and $f_{i}=\widetilde{\sigma_{3}^{n}} t_{1}$ for some $t_{1} \in \mathfrak{T}$ (in fact $t_{1}=t$ because $\left.\widetilde{\sigma_{3}^{n}}={\widetilde{\sigma_{3}}}^{n}\right)$. Then $t_{1} \in \mathfrak{T}^{\langle 3\rangle}$ and as in previous cases $A=A(3, \mathrm{id})$. But this is conjugated to a subgroup of $A(105, \mathrm{id})$ by Corollary 3 .

- We may suppose now that each $\sigma_{j}$ is in the orbit of either $\sigma_{105}$ or $\sigma_{405}$. We prove next that either all the $\sigma_{j}$ 's are in the orbit of $\sigma_{105}$ or $\sigma_{405}$. Otherwise, after re-ordering and applying Corollary 2 we can take $f_{1}=\widetilde{\sigma_{405}}$ and $f_{2}=\widetilde{\sigma_{105}} t$ for some $t \in \mathfrak{T}$. Thus $B \subset \mathfrak{T}^{\langle 105\rangle} \cap \mathfrak{T}^{\langle 405\rangle}$. Moreover $B \subsetneq \widetilde{B}:=\left\langle B \cup\left\{f_{1} f_{2}\right\}\right\rangle \subset A$ and $\widetilde{B} \subset\left\langle\left(\mathfrak{T}^{\langle 105\rangle} \cap \mathfrak{T}^{\langle 405\rangle}\right) \cup\right.$ $\left.\left\{\widetilde{\sigma_{405} \sigma_{105}} t\right\}\right\rangle \subset A\left(1048, t_{1}\right)$ for some $t_{1} \in \mathfrak{T}$, taking into account that $\sigma_{405} \sigma_{105}=\sigma_{1048}$. But $A\left(1048, t_{1}\right)$ is toral since $\sigma_{1048}$ is in the orbit of $\sigma_{142}$ (see Propositions 4 and 5). This contradicts the maximal toral nature of $B$ within $A$.

- If all the $\sigma_{j}$ 's are in the orbit of $\sigma_{405}$, since this orbit has cardinal one, then we can take $f_{1}=\widetilde{\sigma_{405}}$ and any other $f_{i}$ of the form $\widetilde{\sigma_{405}} t$ with $t \in \mathfrak{T}^{\langle 405\rangle}$. So $A \subset A(405$, id $)$.

- If all the $\sigma_{j}$ 's are in the orbit of $\sigma_{105}$. The unique elements in this orbit which commute with $\sigma_{105}$ are $\sigma_{105}, \sigma_{403}, \sigma_{429}$ and $\sigma_{1011}$. So we can take $f_{1}=\widetilde{\sigma_{105}} t_{1}$ and any other $f_{i}$ of the form $\widetilde{\sigma_{k}} t_{2}$ for some $t_{1}, t_{2} \in \mathfrak{T}$ and $k \in\{105,403,429,1011\}$. Then $B \subset \widetilde{B}:=\left\langle B \cup\left\{f_{1} f_{i}\right\}\right\rangle \subset A$ and depending on the values of $k$ we have:

- For $k=403$, since $\sigma_{105} \sigma_{403}=\sigma_{1050}$, we have $f_{1} f_{i}=\widetilde{\sigma_{105}} t_{1} \widetilde{\sigma_{403}} t_{2}=$ $\widetilde{\sigma_{1050}} t$ for some $t \in \mathfrak{T}$. So $\widetilde{B} \subset A(1050, t)$ which is toral because $\sigma_{1050}$ is in the orbit of $\sigma_{103}$ (see again Propositions 4 and 5). This contradicts the maximality of $B$ among the toral subgroups of $A$.

- For $k=429$ we have $\sigma_{105} \sigma_{429}=\sigma_{1009}$ so that $B \subsetneq \widetilde{B} \subset A(1009, t)$ for some $t \in \mathfrak{T}$. This is toral since $\sigma_{1009}$ is conjugated to $\sigma_{103}$ in $\mathcal{W}$. Again a contradiction.

- For $k=1011$ we have $\sigma_{105} \sigma_{1011}=\sigma_{427}$ and $B \subsetneq \widetilde{B} \subset A(427, t)$, for $t \in \mathfrak{T}$. This is again toral because $\sigma_{427}$ is in the orbit of $\sigma_{103}$.

These contradictions lead us to the conclusion that $k=105$ necessarily. So $f_{i}$ is of the form $\widetilde{\sigma_{105}} t_{2}$ and the commutativity of $f_{1}$ and $f_{i}$ implies that $t_{1} t_{2}^{-1} \in \mathfrak{T}^{\langle 105\rangle}$. Hence $f_{i} \in f_{1} \mathfrak{T}^{\langle 105\rangle}$ (for any other $i$ ) implying $A \subset A\left(105, t_{1}\right)$. Thus, after conjugation $A$ is a subgroup of $A(105$,id) by Proposition 6 . 
Corollary 4. Up to equivalence the unique fine gradings on $\mathfrak{f}_{4}$ are: (1) the Cartan grading, (2) the induced by $A(105, i d)$, (3) the induced by $A(405, i d)$, and (4) the induced by $A(15, i d)$.

We summarize the information about these gradings in the following table

\begin{tabular}{|c|c|c|}
\hline Quasitorus & Universal grading group & Type \\
\hline \hline $\mathfrak{T}$ & $\mathbb{Z}^{4}$ & $(48,0,0,1)$ \\
$A(15$, id $)$ & $\mathbb{Z}_{3}^{3}$ & $(0,26)$ \\
$A(105$, id $)$ & $\mathbb{Z}_{2}^{3} \times \mathbb{Z}$ & $(31,0,7)$ \\
$A(405$, id $)$ & $\mathbb{Z}_{2}^{5}$ & $(24,0,0,7)$ \\
\hline
\end{tabular}

Fine gradings on $\mathfrak{f}_{4}$

Let us observe that the groups given in the table above are the universal grading groups. Because for any of the quasitorus $Q$ in the table, we have proved in Proposition 9 that $Q$ is its own centralizer in $\mathfrak{F}_{4}$, that is, $Q$ is a MAD, and according to Subsection 2.1, $\mathfrak{X}(Q)$ is the universal group of the related grading.

Remark 3. The four fine gradings on $\mathfrak{f}_{4}$ are also fine when considered as Lie gradings (as in [37]) instead of being considered as group gradings. This observation is pertinent because the question about the existence of a grading group is still on the table.

On the other hand, we observe that every homogeneous element in the Cartan grading is either semisimple or nilpotent (according to its membership to the zero component) and that all the homogeneous elements in the three remaining fine gradings are semisimple, as a consequence of the nullity of their zero components ([34, Corollary after Th. 3.4]). This happens not only in $\mathfrak{f}_{4}$.

Proposition 10. In a fine grading on a simple Lie algebra, every homogeneous element is either semisimple or nilpotent.

Proof. If $L=\oplus_{g \in G} L_{g}$ is a fine grading, the group $Q$ of the automorphisms of $L$ for which all the homogeneous components are eigensubspaces is just the MAD which produces the grading.

First of all, if $x \in L_{g}$ then $x$ is uniquely represented as $x=x_{s}+x_{n}$, where $x_{s}$ and $x_{n}$ are respectively semisimple and nilpotent homogeneous elements in $L_{g}$ verifying $\left[x_{s}, x_{n}\right]=0$ [34, Th.3.3]. Thus, either every element in $L_{g}$ is semisimple or there is a nilpotent element $x \in L_{g}$. Let us see that in this case every element in $L_{g}$ is nilpotent. According to [34, Th. 3.4], there is a semisimple element $h \in L_{e}$ and a nilpotent element $y \in L_{-g}$ such that 
$[h, x]=2 x,[h, y]=-2 y$ and $[x, y]=h$. Notice then that $\exp \operatorname{ad}(h) \in \operatorname{aut}(L)$ is an automorphism which leaves invariant all the homogeneous components. Moreover, $\exp \operatorname{ad}(h)$ commutes with every $f \in Q$, because if $v$ is a homogeneous element with $f(v)=\alpha v$, then $(\exp \operatorname{ad}(h)) f(v)=\alpha \exp \operatorname{ad}(h)(v)=$ $f(\exp \operatorname{ad}(h)(v))$ since exp $\operatorname{ad}(h)(v)$ belongs to the same homogeneous component. By maximality, $\exp \operatorname{ad}(h) \in Q$. Since exp $\operatorname{ad}(h)(x)=e^{2} x$, there are some automorphisms $f_{1}, \ldots, f_{s} \in Q$ and scalars $\alpha_{1}, \ldots, \alpha_{s} \in F^{\times}$such that $L_{g}=\left\{v \in L \mid f_{1}(v)=\alpha_{1} v, \ldots, f_{s}(v)=\alpha_{s} v, \exp \operatorname{ad}(h)(v)=e^{2} v\right\}$. Therefore $g \in G$ is an element of infinite order, and, by [34, Prop. 3.5], all the elements in $L_{g}$ are nilpotent.

More information on homogeneous semisimple and nilpotent elements of gradings on semisimple Lie algebras can be found in [41]. Notice the following interesting consequence for fine gradings.

Corollary 5. In a fine grading on a simple Lie algebra, the zero homogeneous component is an abelian subalgebra whose dimension coincides with the Betti number of the universal grading group.

Proof. The subalgebra $L_{e}$ (the zero component) is reductive, hence, if $L_{e} \neq 0$, it contains (nonzero) semisimple elements, and applying the previous proposition, every element in $L_{e}$ is semisimple. So that $L_{e}$ is toral and abelian.

If $Q$ is the MAD-group inducing the grading, then $\mathfrak{X}(Q)$ is the universal grading group and its Betti number is the dimension of $Q$, that is, the dimension of the maximal torus contained in $Q$. Note that the torus $T=\exp \operatorname{ad}\left(L_{e}\right)$ preserves the grading, so that it commutes with $Q$ and, by maximality, $T \subset Q$. But $T$ must be a maximal torus inside $Q$, because if $T \subset T^{\prime}=\exp \operatorname{ad}(M) \subset Q$ then $M$ would be a subalgebra invariant by the whole $Q$. Therefore it would be contained in $L_{e}$ and thus $L_{e}=M$ and $T=T^{\prime}$. Consequently $\operatorname{dim} L_{e}=\operatorname{dim} T=\operatorname{dim} Q$.

\subsection{Nontoral gradings}

Up to the moment we have described the fine gradings on $\mathfrak{f}_{4}$. If we want to find all the nontoral ones, it suffices to describe all the nontoral coarsenings of the fine ones. For this, we must find all the (nontoral) subquasitori $Q$ of the tori producing fine gradings up to conjugacy.

Consider first the fine grading provided by $A(105, \mathrm{id})=\left\langle\mathfrak{T}^{\langle 105\rangle} \cup\left\{\widetilde{\sigma_{105}}\right\}\right\rangle$. Let us use the following notations $g_{1}:=t_{-1,1,-1,1}^{\prime}, g_{2}:=t_{1,-1,-1,1}^{\prime}, g_{3}:=\widetilde{\sigma_{105}}$ and $g_{4}:=t_{1,1,1,-1}^{\prime}$. Since the number of generators of the group $H=\mathfrak{X}(Q)$ must be at least three, we know that $H$, as a quotient of $\mathbb{Z}_{2}^{3} \times \mathbb{Z}$, falls under one of the following possibilities: either $H \cong \mathbb{Z}_{2}^{3} \times \mathbb{Z}_{m}$, or $H \cong \mathbb{Z}_{2}^{2} \times \mathbb{Z}_{2 m}$, 
or $H \cong \mathbb{Z}_{2}^{2} \times \mathbb{Z}$. Let us analyze first the case $H \cong \mathbb{Z}_{2}^{3}$. In this case the quasitorus $Q$ providing the coarsening has three order-two generators $\varphi_{i}$ with $i=1,2,3$. Thus $\varphi_{i}=g_{1}^{n_{i}} g_{2}^{m_{i}} g_{3}^{l_{i}} g_{4}^{s_{i}}$ and $\left(\varphi_{1}, \varphi_{2}, \varphi_{3}\right)=\left(g_{1}, g_{2}, g_{3}, g_{4}\right) \cdot M$ where $M$ is the $3 \times 3$ integer matrix

$$
\left(\begin{array}{ccc}
n_{1} & n_{2} & n_{3} \\
m_{1} & m_{2} & m_{3} \\
l_{1} & l_{2} & l_{3} \\
s_{1} & s_{2} & s_{3}
\end{array}\right) .
$$

We are using here the action of $M_{k \times l}(\mathbb{Z})$ on any power group $G^{k}$ given by $G^{k} \times M_{k \times l}(\mathbb{Z}) \rightarrow G^{l}$ such that $\left(g_{1}, \ldots, g_{k}\right) \cdot\left(n_{i j}\right):=\left(g_{1}^{\prime}, \ldots, g_{l}^{\prime}\right)$ where $g_{i}^{\prime}=\prod_{j} g_{j}^{n_{j i}}$. Now, there are two actions that we can use to simplify the matrix $M$ without changing the quasitorus $Q$ (in the worst case $Q$ changes to some of its conjugated). First we can act on the columns of the matrix by elementary operations (exchanging columns or adding one column to another). All this can be made modulo two. This reduces the possible matrices $M$ to a few, but there is a second action on the matrix coming from conjugacy of elements in $\mathfrak{F}_{4}$. Consider the subgroup $G$ of $\mathfrak{F}_{4}$ fixing $A(105$, id) by conjugation, that is, $f \in G$ if and only if $f A(105$, id $) f^{-1}=A(105$, id $)$. The appendix contains relevant information on certain elements in $G$. By passing from $Q$ to $f Q f^{-1} \subset A(105$, id), the matrix $M$ transforms into a new matrix $M^{\prime}$. We are proving that the joint action by elementary operations on the columns together with the action of $G$ by conjugation, either moves $M$ to the matrix

$$
\left(\begin{array}{lll}
1 & 0 & 0 \\
0 & 1 & 0 \\
0 & 0 & 1 \\
0 & 0 & 0
\end{array}\right),
$$

or the induced grading is toral. First we observe that if this grading is nontoral there must be some nonzero entry in the first two rows of $M$. Second, $\widetilde{\sigma_{468}} \in G$ acts on $M$ permuting its first two rows (it permutes $g_{1}$ and $g_{2}$ fixing $g_{i}$ for $\left.i=3,4\right)$. So we can suppose that the first row in $M$ is $\left(\begin{array}{lll}1 & 0 & 0\end{array}\right)$ (after operations on columns). Hence the first two rows are $\left(\begin{array}{lll}1 & 0 & 0 \\ 0 & 1 & 0\end{array}\right),\left(\begin{array}{lll}1 & 0 & 0 \\ 0 & 0 & 0\end{array}\right)$ or $\left(\begin{array}{lll}1 & 0 & 0 \\ 1 & 0 & 0\end{array}\right)$, also after operations on columns. However $\widetilde{\sigma_{94}} \in G$ acting on the second matrix produces the third one. The grading induced by the third matrix is $\left\langle g_{1} g_{2} g_{3}^{n_{1}} g_{4}^{n_{2}}, g_{3}^{m_{1}} g_{4}^{m_{2}}, g_{3}^{l_{1}} g_{4}^{l_{2}}\right\rangle$ where the couples $\left(m_{1}, m_{2}\right)$ and $\left(l_{1}, l_{2}\right)$ are linearly independent in the vector space $\mathbb{Z}_{2}^{2}$ (otherwise the grading has two generators and so is toral). Thus $\left(n_{1}, n_{2}\right)$ is a linear combination of them and the grading can be written as $\left\langle g_{1} g_{2} f^{k} g^{h}, f, g\right\rangle$ where $f, g \in\left\langle g_{3}, g_{4}\right\rangle$. But $\left\langle g_{1} g_{2} f^{k} g^{h}, f, g\right\rangle=\left\langle g_{1} g_{2}, f, g\right\rangle$ hence we have the (agreeing) possibilities $\left\langle g_{1} g_{2}, g_{3}, g_{4}\right\rangle=\left\langle g_{1} g_{2}, g_{3} g_{4}, g_{3}\right\rangle=\left\langle g_{1} g_{2}, g_{3} g_{4}, g_{4}\right\rangle$. So in this case we must only worry about the grading $\left\langle g_{1} g_{2}, g_{3}, g_{4}\right\rangle$, which can be proved to be toral (seen 
as a grading on the Albert algebra, this comes from a necessarily toral $\mathbb{Z}_{2} \times$ $\mathbb{Z}_{2}$-grading on the octonions refined with the $\mathbb{Z}_{2}$-grading on $H_{3}(F)$, see Subsection 3.1, but alternatively we next prove that is conjugated to $\left.\left\langle g_{1}, g_{2}, g_{4}\right\rangle\right)$. The first matrix above produces the grading $\left\langle g_{1} g_{3}^{n_{1}} g_{4}^{n_{2}}, g_{2} g_{3}^{m_{1}} g_{4}^{m_{2}}, g_{3}^{l_{1}} g_{4}^{l_{2}}\right\rangle$. But $\widetilde{\sigma_{485}}$ permutes $g_{1}$ and $g_{2}$ while $\widetilde{\sigma_{485}} g_{3}{\widetilde{\sigma_{485}}}^{-1}=g_{3} g_{4}$ and $\widetilde{\sigma_{485}} g_{4}{\widetilde{\sigma_{485}}}^{-1}=g_{4}$, so we can suppose $l_{1}=1, l_{2}=0$ or $l_{1}=0, l_{2}=1$. This reduces the possibilities to $\left\langle g_{1} g_{4}^{n_{2}}, g_{2} g_{4}^{m_{2}}, g_{3}\right\rangle$ or $\left\langle g_{1} g_{3}^{n_{1}}, g_{2} g_{3}^{m_{1}}, g_{4}\right\rangle$. The four different possibilities for the first case are $\left\langle g_{1}, g_{2}, g_{3}\right\rangle,\left\langle g_{1} g_{4}, g_{2}, g_{3}\right\rangle,\left\langle g_{1}, g_{2} g_{4}, g_{3}\right\rangle,\left\langle g_{1} g_{4}, g_{2} g_{4}, g_{3}\right\rangle$, but conjugating by $\widetilde{\sigma_{103}} \in G$, the first and second gradings turn out to be isomorphic, so as the third and the fourth ones; while conjugation by $\widetilde{\sigma_{468}} \in G$ proves the isomorphism between the second and the third gradings. On the other hand the four possibilities for $\left\langle g_{1} g_{3}^{n_{1}}, g_{2} g_{3}^{m_{1}}, g_{4}\right\rangle$ are $\left\langle g_{1}, g_{2}, g_{4}\right\rangle$ (which is obviously toral), $\left\langle g_{1} g_{3}, g_{2}, g_{4}\right\rangle,\left\langle g_{1}, g_{2} g_{3}, g_{4}\right\rangle$ and $\left\langle g_{1} g_{3}, g_{2} g_{3}, g_{4}\right\rangle$. But conjugation by $\widetilde{\sigma_{468}}$ makes evident the isomorphism between the second and the third gradings while $\left\langle g_{1} g_{3}, g_{2} g_{3}, g_{4}\right\rangle=\left\langle g_{1} g_{2}, g_{2} g_{3}, g_{4}\right\rangle=\left\langle g_{1} g_{2}, g_{1} g_{3}, g_{4}\right\rangle$ and conjugation by $\widetilde{\sigma_{491}} \in G$ proves that this is isomorphic to $\left\langle g_{1} g_{3}, g_{2}, g_{4}\right\rangle$. Now it is possible to show that there exists an element $\psi \in G$ such that $\psi g_{i} \psi^{-1}=g_{i}$ for $i=1,4$ while $\psi g_{2} \psi^{-1}=g_{3}$ and $\psi g_{3} \psi^{-1}=g_{2}$ (the element $\psi$ can be taken of order 4). So $\psi\left\langle g_{1} g_{3}, g_{2}, g_{4}\right\rangle \psi^{-1}=\left\langle g_{1} g_{2}, g_{3}, g_{4}\right\rangle$, whose torality has been previously stated. Moreover, $\left\langle g_{1} g_{2}, g_{3}, g_{4}\right\rangle$ is conjugated to $\left\langle g_{1}, g_{3}, g_{4}\right\rangle$ by $\widetilde{\sigma_{94}}$ and this is conjugated to $\left\langle g_{1}, g_{2}, g_{4}\right\rangle$ by $\psi$. This last one is obviously toral. Summarizing the results in this paragraph we have:

Proposition 11. If $Q \subset A(105, i d)$ is a quasitorus with $\mathfrak{X}(Q) \cong \mathbb{Z}_{2}^{3}$ then it is conjugated to $\left\langle g_{1}, g_{2}, g_{3}\right\rangle$ if $Q$ is nontoral, and to $\left\langle g_{1}, g_{2}, g_{4}\right\rangle$ if it is toral. Moreover the conjugating element can be taken to fix the subgroup $A(105, i d)$. The $\mathbb{Z}_{2}^{3}$-grading induced by $\left\langle g_{1}, g_{2}, g_{3}\right\rangle$ is of type $(0,0,1,0,0,0,7)$.

Proof. We have proved in the previous paragraph that $Q$ is conjugated to either $\left\langle g_{1}, g_{2}, g_{3}\right\rangle$ or $\left\langle g_{1}, g_{2}, g_{4}\right\rangle$. Obviously $\left\langle g_{1}, g_{2}, g_{4}\right\rangle$ is toral. On the other hand, the grading induced by $\left\langle g_{1}, g_{2}, g_{3}\right\rangle$ is nontoral, of type $(0,0,7,0,0,1)$ in the Albert algebra and of type $(0,0,1,0,0,0,7)$ in $\mathfrak{f}_{4}$. Its nontoral character is obvious noticing that the dimension of its 0-homogeneous component in $\mathfrak{f}_{4}$ is 3 .

Proposition 12. If $Q \subset A(105, i d)$ is a nontoral quasitorus such that $\mathfrak{X}(Q) \cong \mathbb{Z}_{2}^{3} \times \mathbb{Z}_{m}$, with $m>1$, then up to conjugacy there is $v \in F^{\times}$a primitive $m$-root of the unit such that $Q=\left\langle g_{1}, g_{2}, g_{3}, t_{1,1,1, v}^{\prime}\right\rangle$.

Proof. The quasitorus $Q=\left\langle\phi_{i}\right\rangle_{i=1}^{4}$ is generated by $\phi_{1}, \phi_{2}, \phi_{3}$ order-two elements in $\mathfrak{F}_{4}$ and $\phi_{4}$ an order- $m$ element in $\mathfrak{F}_{4}$. We can apply the previous proposition to $Q^{\prime}:=\left\langle\phi_{1}, \phi_{2}, \phi_{3}\right\rangle$. So we can assume (by conjugating) that either (1) $Q^{\prime}=\left\langle g_{1}, g_{2}, g_{3}\right\rangle$, or (2) $Q^{\prime}=\left\langle g_{1}, g_{2}, g_{4}\right\rangle$, with $\phi_{4}=g_{1}^{n} g_{2}^{k} g_{3}^{l} t_{1,1,1, u}^{\prime}$ for 
some $n, k, l \in\{0,1\}$ and some $u \in F^{\times}$. From $\phi_{4}^{m}=\mathrm{id}$, it follows that $l m$ is even (either $l$ or $m$ ) and $t_{(-1)^{n},(-1)^{k},(-1)^{n+k}, u}^{\prime}$ has order $m$, in particular $u^{m}=1$.

In case (1),

$$
Q=\left\langle g_{1}, g_{2}, g_{3}, g_{1}^{n} g_{2}^{k} g_{3}^{l} t_{1,1,1, u}^{\prime}\right\rangle=\left\langle g_{1}, g_{2}, g_{3}, t_{1,1,1, u}^{\prime}\right\rangle, \quad \text { but } t_{1,1,1, u}^{\prime} \notin Q^{\prime},
$$

so if $u$ had order $m^{\prime}$ (divisor of $m$ ), $\mathfrak{X}(Q)$ would be isomorphic to $\mathbb{Z}_{2}^{3} \times \mathbb{Z}_{m^{\prime}}$. Hence $u$ is a primitive $m$-root of the unit.

In case (2),

$$
Q=\left\langle g_{1}, g_{2}, g_{4}, g_{1}^{n} g_{2}^{k} g_{3}^{l} t_{1,1,1, u}^{\prime}\right\rangle=\left\langle g_{1}, g_{2}, t_{1,1,1,-1}^{\prime}, g_{3}^{l} t_{1,1,1, u}^{\prime}\right\rangle .
$$

Since $Q$ is nontoral, $l=1$. Hence $m=2 m^{\prime}$ must be even, with $u^{m^{\prime}} \in\{ \pm 1\}$. If $u^{m^{\prime}}=-1,\left(g_{3} t_{1,1,1, u}^{\prime}\right)^{m^{\prime}}$ would be $t_{1,1,1,-1}^{\prime}$ if $m^{\prime}$ is even, and $g_{3} t_{1,1,1,-1}^{\prime}$ if $m^{\prime}$ is odd. In the first case $Q=\left\langle g_{1}, g_{2}, g_{3} t_{1,1,1, u}^{\prime}\right\rangle$, a contradiction with the number of generators of $\mathfrak{X}(Q)$. In the other case, $g_{3} \in Q$, hence $Q=$ $\left\langle g_{1}, g_{2}, t_{1,1,1,-1}^{\prime}, g_{3}, t_{1,1,1, u}^{\prime}\right\rangle=\left\langle g_{1}, g_{2}, g_{3}, t_{1,1,1, u}^{\prime}\right\rangle$ and so $\mathfrak{X}(Q)$ would be a subgroup of $\mathbb{Z}_{2}^{3} \times \mathbb{Z}_{m^{\prime}}$, a contradiction. Thus $u^{m^{\prime}}=1$ and $\left(g_{3} t_{1,1,1, u}^{\prime}\right)^{m^{\prime}}$ would be $t_{1,1,1,1}^{\prime}=$ id if $m^{\prime}$ is even, and $g_{3}$ if $m^{\prime}$ is odd. In the first case again $\mathfrak{X}(G)$ would be a subgroup of $\mathbb{Z}_{2}^{3} \times \mathbb{Z}_{m^{\prime}}$. So that we have the case $m^{\prime}$ odd, in which $g_{3} \in Q$. In this way $Q=\left\langle g_{1}, g_{2}, t_{1,1,1,-1}^{\prime}, g_{3}, t_{1,1,1, u}^{\prime}\right\rangle=\left\langle g_{1}, g_{2}, g_{3}, t_{1,1,1,-u}^{\prime}\right\rangle$, since $\left(t_{1,1,1,-u}^{\prime}\right)^{m^{\prime}}=t_{1,1,1,-1}^{\prime}$. But now $v=-u$ is the required primitive $m$-root of the unit.

Notice that the obtained quasitori $Q=\left\langle g_{1}, g_{2}, g_{3}, t_{1,1,1, v}^{\prime}\right\rangle$ are obviously nontoral because they contain $\left\langle g_{1}, g_{2}, g_{3}\right\rangle$.

The induced gradings by the previous quasitori depend on $m$. For $m=2$ we are talking about a $\mathbb{Z}_{2}^{4}$-grading of type $(1,8,0,0,7)$, for $m=3$ it is a $\mathbb{Z}_{2}^{3} \times \mathbb{Z}_{3}$-grading of type $(3,14,7)$, for $m=4$ it is a $\mathbb{Z}_{2}^{3} \times \mathbb{Z}_{4}$-grading of type $(17,7,7)$, but for $m \geq 5$ all the gradings are equivalent to the one produced by $A(105, \mathrm{id})$, since they are all of type $(31,0,7)$. In general two gradings having the same type are not necessarily equivalent, but of course they are equivalent if the quasitorus producing one of the gradings is contained in the other one, since the homogeneous components of the former are pieces of the homogeneous components of the latter.

Remark 4. If $Q^{\prime} \subset Q$ are quasitori whose induced gradings are of the same type, then these gradings are equivalent.

To continue the study of nontoral gradings coming from subquasitori of $A(105$,id) we must analyze those quasitori $Q \subset A(105$, id) with $\mathfrak{X}(Q) \cong$ $\mathbb{Z}_{2}^{2} \times \mathbb{Z}_{m}$, where $m$ must be even (otherwise $Q$ would have two generators and the grading would be toral). Moreover $m$ must be a multiple of 4 , because we have already studied the cases $m=2$ in Proposition 11 and $m=2 m^{\prime}$ with $m^{\prime}$ odd in Proposition 12. 
Proposition 13. If $Q \subset A(105, i d)$ is a nontoral quasitorus such that $\mathfrak{X}(Q) \cong \mathbb{Z}_{2}^{2} \times \mathbb{Z}_{4 m}$ with $m \geq 1$, then up to conjugacy there is $v \in F^{\times} a$ primitive $4 m$-root of the unit such that $Q=\left\langle g_{1}, g_{2}, g_{3} t_{1,1,1, v}^{\prime}\right\rangle$.

Proof. Suppose that $\left\{\phi_{1}, \phi_{2}, \phi_{3}\right\}$ is a set of generators of $Q$ with $\phi_{1}$ and $\phi_{2}$ of order two and $\phi_{3}$ of order $2 k=4 \mathrm{~m}$. Then $\phi_{3}^{k}$ is an order-two element and we can suppose (by conjugating if necessary) that the subgroup $\left\langle\phi_{1}, \phi_{2}, \phi_{3}^{k}\right\rangle$ is either $\left\langle g_{1}, g_{2}, g_{3}\right\rangle$ or $\left\langle g_{1}, g_{2}, g_{4}\right\rangle$, according to Proposition 11. Besides, $\phi_{3}=$ $g_{1}^{n} g_{2}^{m} g_{3}^{l} t_{1,1,1, u}^{\prime}$ for some $n, m, l \in\{0,1\}$ and $u \in F^{\times}$. As $k$ is even, $\phi_{3}^{k}=$ $t_{1,1,1, u^{k}}^{\prime}$. Since $\phi_{3}^{k}$ has exactly order two, $u^{k}=-1$ and $\phi_{3}^{k}=g_{4}$.

But $\left\langle\phi_{1}, \phi_{2}, \phi_{3}^{k}\right\rangle=\left\langle g_{1}, g_{2}, g_{3}\right\rangle$ is not possible, since $g_{4} \notin\left\langle g_{1}, g_{2}, g_{3}\right\rangle$, so necessarily $\left\langle\phi_{1}, \phi_{2}, \phi_{3}^{k}\right\rangle=\left\langle g_{1}, g_{2}, g_{4}\right\rangle$. Thus

$$
Q=\left\langle\phi_{1}, \phi_{2}, \phi_{3}^{k}, \phi_{3}\right\rangle=\left\langle g_{1}, g_{2}, g_{4}, g_{3}^{l} t_{1,1,1, u}^{\prime}\right\rangle
$$

and $l=1$ since $Q$ is nontoral. But $\left(g_{3} t_{1,1,1, u}^{\prime}\right)^{k}=g_{4}$ so that $Q$ agrees with the group $\left\langle g_{1}, g_{2}, g_{3} t_{1,1,1, u}^{\prime}\right\rangle$, where $u$ has order $2 k=4 m$.

Notice that in this case all the $\mathbb{Z}_{2}^{3}$-coarsenings of the gradings are toral. In spite of that, these gradings are nontoral, because the dimension of the zero homogeneous component is 3 in case $m=1$ and 1 in the remaining cases. If $m=1$, we obtain a $\mathbb{Z}_{2}^{2} \times \mathbb{Z}_{4}$-grading of type $(0,8,2,0,6)$, if $m=2$ we have a

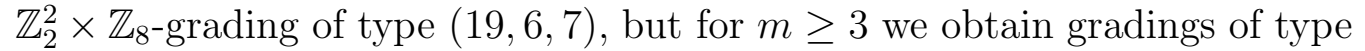
$(31,0,7)$, so equivalent to the fine $\mathbb{Z}_{2}^{3} \times \mathbb{Z}$-grading, again by the previous remark.

Finally we analyze the case $\mathfrak{X}(Q)=\mathbb{Z}_{2}^{2} \times \mathbb{Z}$. The quasitorus $Q$ must be the direct product of a one-dimensional subtorus $P$ of $A\left(105\right.$, id) times $\left\langle\phi_{1}, \phi_{2}\right\rangle$, with $\phi_{i}$ two elements of order two. But necessarily $P=\left\{t_{1,1,1, u}^{\prime} \mid u \in F^{\times}\right\}$ (it is the unique nontrivial subtorus of $A(105, \mathrm{id})$ ). Changing the generators if necessary (to remove $g_{4}$ ), we can write $\left(\phi_{1}, \phi_{2}\right)=\left(g_{3}, g_{2}, g_{1}\right) M$ where $M$ is a $3 \times 2$ matrix with entries in $\mathbb{Z}_{2}$. The first row is nonzero because otherwise the grading would be toral, and performing column operations we can suppose that it is $(10)$. Besides there must be some 1 in each column. As $\widetilde{\sigma_{468}}$ interchanges the second and third row, by doing column operations, the first two rows in $M$ can be taken to be $\left(\begin{array}{ll}1 & 0 \\ 0 & 1\end{array}\right)$. Thus either $Q=P\left\langle g_{3} g_{1}, g_{2}\right\rangle$, or $Q=P\left\langle g_{3}, g_{1} g_{2}\right\rangle$, or $Q=P\left\langle g_{3} g_{1}, g_{2} g_{1}\right\rangle$. By applying the element $\widetilde{\sigma_{491}}$ to the third one, we obtain $P\left\langle g_{1} g_{2} g_{3} g_{4}, g_{2} g_{4}\right\rangle=P\left\langle g_{1} g_{3}, g_{2} g_{4}\right\rangle=P\left\langle g_{1} g_{3}, g_{2}\right\rangle$, the first quasitorus. This one is conjugated to the second one by means of $\psi$ (see Appendix). Besides we can replace $g_{1} g_{2}$ by $g_{1}$ by using $\widetilde{\sigma_{94}}$. Summarizing these facts we have:

Proposition 14. If $Q \subset A(105, i d)$ is nontoral and $\mathfrak{X}(Q) \cong \mathbb{Z}_{2}^{2} \times \mathbb{Z}$ then $Q$ is conjugated to $\left\langle g_{1}, g_{3}, t_{111 u}^{\prime} \mid u \in F^{\times}\right\rangle$. 
Notice that the induced grading on $\mathfrak{f}_{4}$ is of type $(31,0,7)$, so it is again equivalent to the one produced by the whole $A(105$, id).

The following step is to examine the proper subquasitori of $A(15, \mathrm{id})$. However all of them are toral since $A\left(15\right.$,id) is isomorphic to $\mathbb{Z}_{3}^{3}$ hence any proper subquasitorus has a system of generators of cardinal $\leq 2$ (Lemma 2 ).

Thus, to finish our classification of subquasitori of the maximal quasitori, we must analyze $A\left(405\right.$, id). This group is isomorphic to $\mathbb{Z}_{2}^{5}$. Indeed we have $A(405, \mathrm{id})=\left\langle\mathfrak{T}^{\langle 405\rangle} \cup\left\{\widetilde{\sigma_{405}}\right\}\right\rangle$ where $\mathfrak{T}^{\langle 405\rangle}=\left\{t_{x y z u}^{\prime} \mid x^{2}=y^{2}=z^{2}=u^{2}=1\right\} \cong$ $\mathbb{Z}_{2}^{4}$ and $\sigma:=\widetilde{\sigma_{405}}$ is an order two element. Consider a subquasitorus $Q \subset$ $A(405, \mathrm{id})$ with three order-two generators. If $Q$ is nontoral, then $\sigma t \in Q$ for some order-two $t \in \mathfrak{T}$. But applying Corollary 2 we can conjugate $Q$ to some new quasitorus $Q^{\prime}$ with $\sigma \in Q^{\prime}$. Thus we can suppose from the beginning that $\sigma \in Q$, and by elementary operations we can take $Q=\left\langle\sigma, t_{1}, t_{2}\right\rangle$ where $t_{i} \in \mathfrak{T}$ are order-two elements.

It is known that there are two conjugacy classes of elements of order 2 in $\mathfrak{F}_{4}$, related to the diagrams obtained by removing the nodes marked with the number 2 in the affine Dynkin diagram [29, Ch. 8]. The first automorphism, related to the marked diagram:

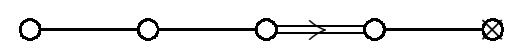

fixes a subalgebra of type $\mathfrak{b}_{4}$, of dimension 36 , and the second one, related to the marked diagram:

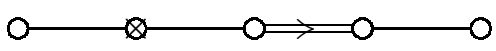

fixes a subalgebra of type $\mathfrak{c}_{3} \oplus \mathfrak{a}_{1}$, of dimension 24 . But if two elements in a maximal torus are conjugated, they are conjugated inside the normalizer of the maximal torus. This means that the Weyl group acts on $\mathfrak{T}^{\langle 405\rangle}$ producing two orbits, apart of the trivial one, characterized by the fact that the dimensions of their fixed parts (the number of 1's in the list (4.4)) are 36 and 24, respectively. This could have been checked by a computer calculation of the action of $\mathcal{W}$ on the set of order-two elements in $\mathfrak{T}$. In the first orbit there are three elements, $t_{1,1,1,-1}^{\prime}, t_{-1,-1,-1,-1}^{\prime}$ and $t_{-1,-1,-1,1}^{\prime}$, and in the second orbit the remaining 12 elements. Thus we can move the element $t_{1}$ in $Q$, since not only $\sigma_{405}=-$ id clearly commutes with any element in $\mathcal{W}$, but $\sigma \widetilde{\sigma}_{j}=\widetilde{\sigma}_{j} \sigma$ for any $j \in\{1, \ldots 1152\}$. Therefore the possibilities for $Q$ are (1) $Q=\left\langle\sigma, t_{-1,1,1,1}^{\prime},-\right\rangle$ and (2) $Q=\left\langle\sigma, t_{1,1,1,-1}^{\prime},-\right\rangle$, as $t_{-1,1,1,1}^{\prime}$ and $t_{1,1,1,-1}^{\prime}$ are representatives of the two orbits. In the first case, the third element can be computed by considering the subgroup of $\mathcal{W}$ fixing $t_{-1,1,1,1}^{\prime}$ and the orbits it produces on the set of order-two elements different from $t_{-1,1,1,1}^{\prime}$. Indeed, that subgroup produces three orbits such that any order-two element differ- 
ent from $t_{-1,1,1,1}^{\prime}$ is conjugated to either $t_{1,-1,1,1}^{\prime}$, or $t_{1,1,1,-1}^{\prime}$ or $t_{1,-1,-1,-1}^{\prime}$ by an element in $\mathcal{W}$ fixing $t_{-1,1,1,1}^{\prime}$. As a consequence the possibilities for $Q$ are $\left\langle\sigma, t_{-1,1,1,1}^{\prime}, t_{1,-1,1,1}^{\prime}\right\rangle,\left\langle\sigma, t_{-1,1,1,1}^{\prime}, t_{1,1,1,-1}^{\prime}\right\rangle$ and $\left\langle\sigma, t_{-1,1,1,1}^{\prime}, t_{1,-1,-1,-1}^{\prime}\right\rangle$. But the second and third of these are conjugated and toral ( $\widetilde{\sigma_{69}}$ relates both of them), hence the unique nontoral $Q$ is, up to conjugacy, $Q=\left\langle\sigma, t_{-1,1,1,1}^{\prime}, t_{1,-1,1,1}^{\prime}\right\rangle$. In the case (2), we can take the third element $t_{2}$ also in the orbit of $t_{1,1,1,-1}^{\prime}$ and so $Q=\left\langle\sigma, t_{1,1,1,-1}^{\prime}, t_{-1,-1,-1,1}^{\prime}\right\rangle$, which produces a toral grading again. It is obviously not conjugated to the previous toral one, because the number of order-two elements in a determined orbit is preserved by conjugation (besides the zero homogeneous components have different dimensions).

Proposition 15. The unique proper subquasitorus of $A(405, i d)$ of order 8 (up to conjugacy) are:

- $\left\langle\widetilde{\sigma_{405}}, t_{-1,1,1,1}^{\prime}, t_{1,-1,1,1}^{\prime}\right\rangle$, inducing a nontoral $\mathbb{Z}_{2}^{3}$-grading of type $(0,0,1$, $0,0,0,7)$.

- $\left\langle\widetilde{\sigma_{405}}, t_{-1,1,1,1}^{\prime}, t_{1,1,1,-1}^{\prime}\right\rangle$, which is toral.

- $\left\langle\widetilde{\sigma_{405}}, t_{1,1,1,-1}^{\prime}, t_{-1,-1,-1,1}^{\prime}\right\rangle$, which is also toral.

The gradings induced by the last two quasitori are not isomorphic.

To finish this subsection we should now describe the nontoral subquasitori $Q$ of $A(405, \mathrm{id})$ isomorphic to $\mathbb{Z}_{2}^{4}$. For this we take any subquasitori $Q^{\prime}$ of $Q$ of cardinal 8, that is, isomorphic to $\mathbb{Z}_{2}^{3}$ and apply the previous study to it. Thus we should study the possible refinements of the quasitori given in Proposition 15 which give nontoral gradings. The techniques already used in the previous paragraph give that any such quasitori is conjugated to $\left\langle\sigma, t_{-1,1,1,1}^{\prime}, t_{1,-1,1,1}^{\prime}, t_{1,1,1,-1}^{\prime}\right\rangle$, which gives a $\mathbb{Z}_{2}^{4}$-grading of type $(1,8,0,0,7)$. The agreement with the type of the $\mathbb{Z}_{2}^{4}$-quasitorus contained in $A(105$, id $)$ suggests that they could be conjugated and the corresponding gradings isomorphic. Indeed, up to conjugacy there is only one abelian nontoral subgroup of $F_{4}$ isomorphic to $\mathbb{Z}_{2}^{3}$ and only one to $\mathbb{Z}_{2}^{4}$ (see for instance [40, Prop. 3.2]). For completeness and selfcontainedness we prove this fact in our context.

Proposition 16. Any nontoral proper subquasitorus of $A(405, i d)$ is conjugated to a subquasitorus of $A(105, i d)$.

Proof. Notice that for $f_{1}=t_{-1,1,-1,1}^{\prime}, f_{2}=t_{1,-1,-1,1}^{\prime}$ and $f_{3}=t_{1,1,1,-1}^{\prime}$, both $\widetilde{\sigma_{105}}$ and $\widetilde{\sigma_{405}}$ commute with the subgroup $\mathfrak{T}^{\langle 105\rangle} \cap \mathfrak{T}^{\langle 405\rangle}=\left\langle f_{1}, f_{2}, f_{3}\right\rangle \cong \mathbb{Z}_{2}^{3}$. In fact both gradings induced by $\left\langle f_{1}, f_{2}, \widetilde{f_{3}}, \widetilde{\sigma_{105}}\right\rangle$ and $\left\langle f_{1}, f_{2}, f_{3}, \widetilde{\sigma_{405}}\right\rangle$ are nontoral. The key fact is that $\left\langle f_{1}, f_{2}, f_{3}, \widetilde{\sigma_{105}} \widetilde{\sigma_{405}}\right\rangle$ is toral (since the fixed part is a four-dimensional abelian subalgebra), so we can apply Proposition 7 
to $F=\left\{f_{i}\right\}_{i=1}^{5}$, for $f_{4}=\widetilde{\sigma_{105}} \widetilde{\sigma_{405}}$ and $f_{5}=\widetilde{\sigma_{105}}$. The group $\langle F\rangle$ is obviously nontoral since it is isomorphic to $\mathbb{Z}_{2}^{5}$. As in the proof of Proposition 7, there is $p \in \mathfrak{F}_{4}$ such that $p f_{i} p^{-1} \in \mathfrak{T}$ for $i=1, \ldots, 4$ and $p f_{5} p^{-1} \in \mathfrak{N}$, that is, there are $j \in\{1, \ldots, 1152\}$ and $t \in \mathfrak{T}$ such that $p f_{5} p^{-1}=\widetilde{\sigma}_{j} t$. As $\mathbb{Z}_{2}^{4} \cong\left\langle p f_{i} p^{-1} \mid i=1 \ldots 4\right\rangle \subset \mathfrak{T}^{\langle j\rangle}$, we have either $j=405$ or $j=748$. But if we had this last possibility, the grading $\left\langle p f_{i} p^{-1} \mid i=1 \ldots 5\right\rangle$ would be toral. Hence $p f_{5} p^{-1}=\widetilde{\sigma_{405}} t$. Moreover $p$ can be taken such that $p f_{5} p^{-1}=\widetilde{\sigma_{405}}$, by Corollary 2, so that $p\langle F\rangle p^{-1} \subset A(405$, id).

Thus, the unique (up to conjugation) nontoral subquasitorus of $A(105$, id) isomorphic to $\mathbb{Z}_{2}^{4}$, which is $\left\langle f_{1}, f_{2}, f_{3}, f_{5}\right\rangle$ according to Proposition 12 , is conjugated by means of $p$ to a subquasitorus of $A(405, \mathrm{id})$, and consequently, the same can be said about the $\mathbb{Z}_{2}^{3}$-nontoral quasitorus $\left\langle f_{1}, f_{2}, f_{5}\right\rangle$. This finishes the proof because there are only two nontoral proper subquasitori of $A(405$, id), by Proposition 15 and the subsequent paragraph.

Summarizing the previous propositions, we have proved the following theorem, which describes all the nontoral quasitori of $\mathfrak{F}_{4}$.

Theorem 5. Any nontoral subquasitorus of $\mathfrak{F}_{4}$ is conjugated to some of the following:

I) $A(15, i d)=\left\langle\widetilde{\sigma_{15}}, t_{\omega, \omega, 1, \omega^{2}}^{\prime}, t_{1, \omega, \omega, 1}^{\prime}\right\rangle \cong \mathbb{Z}_{3}^{3}$, where $\omega$ is a primitive cubic root of 1 .

II) $A(105, i d)=\left\langle\left\{\widetilde{\sigma_{105}}, t_{-1,1,-1,1}^{\prime}, t_{1,-1,-1,1}^{\prime}\right\} \cup\left\{t_{111 u}^{\prime}\right\}_{u \in F^{\times}}\right\rangle \cong F^{\times} \times \mathbb{Z}_{2}^{3}$, and its nontoral (proper) coarsenings which up to conjugacy are:

II.1) $\left\langle\widetilde{\sigma_{105}}, t_{-1,1,-1,1}^{\prime}, t_{1,-1,-1,1}^{\prime}\right\rangle \cong \mathbb{Z}_{2}^{3}$.

II.2) $\left\langle\widetilde{\sigma_{105}}, t_{-1,1,-1,1}^{\prime}, t_{1,-1,-1,1}^{\prime}, t_{1,1,1,-1}^{\prime}\right\rangle \cong \mathbb{Z}_{2}^{4}$.

II.3) $\left\langle\widetilde{\sigma_{105}}, t_{-1,1,-1,1}^{\prime}, t_{1,-1,-1,1}^{\prime}, t_{1,1,1, \rho}^{\prime}\right\rangle \cong \mathbb{Z}_{2}^{3} \times \mathbb{Z}_{m}$, where $m>2$ and $\rho \in F^{\times}$of order $m$.

II.4) $\left\langle t_{-1,1,-1,1}^{\prime}, t_{1,-1,-1,1}^{\prime}, \widetilde{\sigma_{105}} t_{1,1,1, \rho}^{\prime}\right\rangle \cong \mathbb{Z}_{2}^{2} \times \mathbb{Z}_{4 m}$, where $\rho \in F^{\times}$is of order $4 m$.

II.5) $\left\langle\left\{\widetilde{\sigma_{105}}, t_{-1,1,-1,1}^{\prime}\right\} \cup\left\{t_{111 u}^{\prime}\right\}_{u \in F^{\times}}\right\rangle \cong F^{\times} \times \mathbb{Z}_{2}^{2}$.

III) $A(405, i d)=\left\langle\widetilde{\sigma_{405}}, t_{-1,1,1,1}^{\prime}, t_{1,-1,1,1}^{\prime}, t_{1,1,-1,1}^{\prime}, t_{1,1,1,-1}^{\prime}\right\rangle \cong \mathbb{Z}_{2}^{5}$. Its nontoral proper subquasitori are conjugated to the ones in II.1) and II.2).

Therefore, the following table gives all the nontoral gradings on $\mathfrak{f}_{4}$ up to equivalence. We have avoided overlapping of cases produced by the infinite families of quasitori arising in the theorem (computed after Propositions 12, 13 and 14). We give the quasitori, the universal grading groups and the types: 


\begin{tabular}{|c|c|c|c|}
\hline Grading & Group & Type & \\
\hline I & $\mathbb{Z}_{3}^{3}$ & $(0,26)$ & Fine \\
II & $\mathbb{Z}_{2}^{3} \times \mathbb{Z}$ & $(31,0,7)$ & Fine \\
II.1 & $\mathbb{Z}_{2}^{3}$ & $(0,0,1,0,0,0,7)$ & \\
II.2 & $\mathbb{Z}_{2}^{4}$ & $(1,8,0,0,7)$ & \\
II.3.1 & $\mathbb{Z}_{2}^{3} \times \mathbb{Z}_{3}$ & $(3,14,7)$ & \\
II.3.2 & $\mathbb{Z}_{2}^{3} \times \mathbb{Z}_{4}$ & $(17,7,7)$ & \\
II.4.1 & $\mathbb{Z}_{2}^{2} \times \mathbb{Z}_{4}$ & $(0,8,2,0,6)$ & \\
II.4.2 & $\mathbb{Z}_{2}^{2} \times \mathbb{Z}_{8}$ & $(19,6,7)$ & \\
III & $\mathbb{Z}_{2}^{5}$ & $(24,0,0,7)$ & Fine \\
\hline
\end{tabular}

Remark 5. We should notice that there are 9 different equivalence classes of nontoral gradings on $\mathfrak{f}_{4}$. But there are only 8 different ones on the Albert algebra $J$. The device for translating gradings from $J$ to $\mathfrak{f}_{4}$ (and conversely) has this deficiency. However it works well when applied to fine gradings, because MAD's are preserved by the adjoint map. This explains the agreement in the number of fine gradings (up to equivalence).

Remark 6. Theorem 4 states that every quasitorus in $\mathfrak{F}_{4}$ is contained in some $A(j, \mathrm{id})$. Thus every nontoral grading is induced by a set of automorphisms formed by one element in the extension of the Weyl group together with several elements in $\mathfrak{T}$. This result is also true in the context of nontoral gradings on $\mathfrak{g}_{2}$. But it is false when applied to other simple Lie algebras, for instance $\mathfrak{d}_{4}=\mathrm{o}(8, F)$ (see [14]). Then, an alternative approach in the study of nontoral gradings is to find "the first steps", that is, the minimal nontoral quasitori instead of the maximal ones. Independently of the simple Lie algebra under study, they are contained in some $A(j$, id $)$ and any nontoral grading can be obtained by refining one of the gradings produced by them. In the case of $\mathfrak{f}_{4}$, we have found three nontoral minimal quasitori, namely, I, II.1 and II.4.1 in Theorem 5 , which provide $\mathbb{Z}_{3}^{3}, \mathbb{Z}_{2}^{3}$ and $\mathbb{Z}_{2}^{2} \times \mathbb{Z}_{4}$-gradings respectively.

\section{Gradings on the Albert algebra revisited}

In this section we prove Theorem 3. We start by considering the quasitorus $Q$ inducing a nontoral grading on the Albert algebra $J$. The key tool here is the previously mentioned fact that the automorphism group $F_{4}=\operatorname{aut}(J)$ and the automorphism group $\mathfrak{F}_{4}=\operatorname{aut}\left(\mathfrak{f}_{4}\right)$ are isomorphic via the map Ad: $F_{4} \rightarrow \mathfrak{F}_{4}$ such that $\operatorname{Ad}(f) d:=f d f^{-1}$ for any $f \in F_{4}$ and $d \in \mathfrak{f}_{4}$ ([27]). Then we can apply Theorem 5 to have an immediate view (up to conjugacy) of the quasitory $Q$. So we study the different possibilities I)-III) provided by Theorem 5 and collect them in the following table 


\begin{tabular}{|c|c|c|c|}
\hline Grading & Group & Type & \\
\hline I & $\mathbb{Z}_{3}^{3}$ & $(27)$ & Fine \\
II & $\mathbb{Z}_{2}^{3} \times \mathbb{Z}$ & $(25,1)$ & Fine \\
II.1 & $\mathbb{Z}_{2}^{3}$ & $(0,0,7,0,0,1)$ & \\
II.2 & $\mathbb{Z}_{2}^{4}$ & $(7,8,0,1)$ & \\
II.3.1 & $\mathbb{Z}_{2}^{3} \times \mathbb{Z}_{3}$ & $(21,3)$ & \\
II.3.2 & $\mathbb{Z}_{2}^{3} \times \mathbb{Z}_{4}$ & $(23,2)$ & \\
II.4 & $\mathbb{Z}_{2}^{2} \times \mathbb{Z}_{4}$ & $(6,9,1)$ & \\
III & $\mathbb{Z}_{2}^{5}$ & $(24,0,1)$ & Fine \\
\hline
\end{tabular}

It is remarkable the fact that all the quasitori in case II. 4 of Theorem 5 induce the same grading on $J$ up to equivalence (by Remark 4). This was not the case in $\mathfrak{f}_{4}$ where these quasitori produced two nonequivalent gradings. This is the reason why we only have eight equivalence classes of nontoral gradings on $J$ while in $\mathfrak{f}_{4}$ we have detected nine.

Now, any nontoral grading on $J$ must be equivalent to any of these, and these are nonequivalent. But the gradings (3.7), (3.8), (3.9), (3.10), (3.11), (3.1), (3.12) and (3.13) described in Theorem 3 are nonequivalent since their types are different:

\begin{tabular}{|c|c|}
\hline Grading & Type \\
\hline$(3.7)$ & $(25,1)$ \\
$(3.8)$ & $(7,8,0,1)$ \\
$(3.9)$ & $(21,3)$ \\
\hline
\end{tabular}$\quad$\begin{tabular}{cc|}
\hline Grading & Type \\
\hline$(3.10)$ & $(23,2)$ \\
$(3.11)$ & $(24,0,1)$ \\
$(3.1)$ & $(0,0,7,0,0,1)$ \\
\hline
\end{tabular}

\begin{tabular}{|c|c|}
\hline Grading & Type \\
\hline$(3.12)$ & $(6,9,1)$ \\
$(3.13)$ & $(27)$ \\
\hline
\end{tabular}

and so they give a complete system of pairwise nonequivalent nontoral gradings on the Albert algebra.

Remark 7. There is only one equivalence class of group gradings on $J$ with every nonzero component spanned by an invertible element, namely the $\mathbb{Z}_{3} \times \mathbb{Z}_{3} \times \mathbb{Z}_{3}$-grading (19). Thus $J$ is a Jordan $\Lambda$-torus, according to [5, Remark 9.2.1]. Although Jordan tori have been classified in [42], Theorem 3 provides an alternative proof of the uniqueness.

\section{Description of the nontoral gradings on $\mathfrak{f}_{4}$}

In this section we would like to give a more detailed description of the fine gradings on $\mathfrak{f}_{4}$. This description is going to be twofold. On the one hand by using any software allowing simultaneous diagonalization we can get the homogeneous spaces of the grading under consideration in terms of the basis introduced in 4.1. We include this description for its possible use in applications requiring explicit computations. For instance, the subject of gradings is closely related to that of graded contractions [32]. In the setting of graded contractions, new Lie algebras are obtained by modifying the commuting relations preserving the grading. 
But on the other hand we would like to highlight the fact that the whole algebraic group stuff used in this work has been needed to prove that we have captured all the gradings. However this is not necessary at all to describe these gradings. This can be made in an independent way and this is why in this section we are giving natural descriptions of all the fine gradings. Of course the term natural is used here in a subjective manner meaning that the mere description of the gradings (which follows) needs no computer methods neither involves the Weyl group or maximal tori. Thus any mathematician could check the gradings ignoring such tools.

\section{1. $\mathbb{Z}_{3}^{3}$-grading on $\mathfrak{f}_{4}$}

This grading has been previously referenced in the literature though mostly in geometry rather than in algebra. Geometers have studied elementary $p$ groups with different purposes other than the study of gradings. The fine $\mathbb{Z}_{3}^{3}$ grading appears for instance in [19, THEOREM 11.13] from the viewpoint of compact Lie groups but $[3,8.1]$ gives results showing how to translate the arguments to the algebraic groups setting. On the other hand this $\mathbb{Z}_{3^{-}}^{3}$ grading has been studied from the viewpoint of Jordan groups (see [34, p. 127]). It was Alekseevskij, in [4, Table 1], who classified Jordan subgroups in the exceptional case.

The description of the gradings in terms of root vectors can be obtained instantaneously by performing a simultaneous diagonalization of $\mathfrak{f}_{4}$ relative to the set of automorphisms $\left\{\widetilde{\sigma_{15}}, t_{\omega, \omega, 1, \omega^{2}}^{\prime}, t_{1, \omega, \omega, 1}^{\prime}\right\}$. Thus we obtain the following fine $\mathbb{Z}_{3}^{3}$-grading of type $(0,26)$ on $L=\mathfrak{f}_{4}$ :

$$
\begin{aligned}
& L_{000}=0 \\
& L_{001}=\left\langle b_{17}+b_{32}+b_{33},-b_{22}+b_{34}+b_{42}\right\rangle \\
& L_{002}=\left\langle b_{8}+b_{9}+b_{41}, b_{10}+b_{18}+b_{46}\right\rangle \\
& L_{010}=\left\langle b_{1}+b_{2}+b_{29}, b_{4}-b_{23}+b_{48}\right\rangle \\
& L_{011}=\left\langle-b_{19}+b_{30}+b_{38},-b_{20}+b_{31}+b_{39}\right\rangle \\
& L_{012}=\left\langle b_{3}+b_{13}+b_{40}, b_{11}-b_{12}+b_{45}\right\rangle \\
& L_{020}=\left\langle b_{5}+b_{25}+b_{26},-b_{24}-b_{28}+b_{47}\right\rangle \\
& L_{021}=\left\langle-b_{21}-b_{35}+b_{36}, b_{16}-b_{27}+b_{37}\right\rangle \\
& L_{022}=\left\langle-b_{6}-b_{14}+b_{43}, b_{7}-b_{15}+b_{44}\right\rangle \\
& L_{100}=\left\langle t_{\alpha_{1}}+(2+\omega) t_{\alpha_{2}}+2 t_{\alpha_{3}},\left((-1-\omega) t_{\alpha_{1}}\right)+(-1-2 \omega) t_{\alpha_{2}}+2 t_{\alpha_{4}}\right\rangle, \\
& L_{101}=\left\langle\omega b_{17}+\omega^{2} b_{32}+b_{33},-\omega^{2} b_{22}+\omega b_{34}+b_{42}\right\rangle \\
& L_{102}=\left\langle\omega b_{8}+\omega^{2} b_{9}+b_{41}, \omega^{2} b_{10}+\omega b_{18}+b_{46}\right\rangle \\
& L_{110}=\left\langle\omega b_{1}+\omega^{2} b_{2}+b_{29}, \omega b_{4}-\omega^{2} b_{23}+b_{48}\right\rangle \\
& L_{111}=\left\langle-\omega^{2} b_{19}+\omega b_{30}+b_{38},-\omega b_{20}+\omega^{2} b_{31}+b_{39}\right\rangle \\
& L_{112}=\left\langle\omega^{2} b_{3}+\omega b_{13}+b_{40}, \omega b_{11}-\omega^{2} b_{12}+b_{45}\right\rangle \\
& L_{120}=\left\langle\omega b_{5}+\omega^{2} b_{25}+b_{26},-\omega b_{24}-\omega^{2} b_{28}+b_{47}\right\rangle
\end{aligned}
$$




$$
\begin{aligned}
& L_{121}=\left\langle-\omega b_{21}-\omega^{2} b_{35}+b_{36}, \omega^{2} b_{16}-\omega b_{27}+b_{37}\right\rangle, \\
& L_{122}=\left\langle-\omega^{2} b_{6}-\omega b_{14}+b_{43}, \omega b_{7}-\omega^{2} b_{15}+b_{44}\right\rangle, \\
& L_{200}=\left\langle t_{\alpha_{1}}+\left(2+\omega^{2}\right) t_{\alpha_{2}}+2 t_{\alpha_{3}},\left(-1-\omega^{2}\right) t_{\alpha_{1}}+\left(-1-2 \omega^{2}\right) t_{\alpha_{2}}+2 t_{\alpha_{4}}\right\rangle, \\
& L_{201}=\left\langle\omega^{2} b_{17}+\omega b_{32}+b_{33},-\omega b_{22}+\omega^{2} b_{34}+b_{42}\right\rangle, \\
& L_{202}=\left\langle\omega^{2} b_{8}+\omega b_{9}+b_{41}, \omega b_{10}+\omega^{2} b_{18}+b_{46}\right\rangle, \\
& L_{210}=\left\langle\omega^{2} b_{1}+\omega b_{2}+b_{29}, \omega^{2} b_{4}-\omega b_{23}+b_{48}\right\rangle \\
& L_{211}=\left\langle-\omega b_{19}+\omega^{2} b_{30}+b_{38},-\omega^{2} b_{20}+\omega b_{31}+b_{39}\right\rangle, \\
& L_{212}=\left\langle\omega b_{3}+\omega^{2} b_{13}+b_{40}, \omega^{2} b_{11}-\omega b_{12}+b_{45}\right\rangle, \\
& L_{220}=\left\langle\omega^{2} b_{5}+\omega b_{25}+b_{26},-\omega^{2} b_{24}-\omega b_{28}+b_{47}\right\rangle, \\
& L_{221}=\left\langle-\omega^{2} b_{21}-\omega b_{35}+b_{36}, \omega b_{16}-\omega^{2} b_{27}+b_{37}\right\rangle, \\
& L_{222}=\left\langle-\omega b_{6}-\omega^{2} b_{14}+b_{43}, \omega^{2} b_{7}-\omega b_{15}+b_{44}\right\rangle .
\end{aligned}
$$

The easiest way to visualize this grading intrinsically, that is, with no reference to a particular basis or computer methods, may be considering Lie algebra models based upon $\mathbb{Z}_{3}$-gradings. Perhaps the most natural place where to look at the automorphisms inducing the grading is the Lie algebra $\mathfrak{e}_{6}$. Adams gave a construction of this algebra from three copies of $\mathfrak{a}_{2}$ $([1$, p. 85]). This model has been widely spread for its nice 3 -symmetry. Once the automorphisms have been given in $\mathfrak{e}_{6}$ we could hopefully restrict them to $\mathfrak{f}_{4}$. Given a three-dimensional $F$-vector space $X$ in which a nonzero alternating trilinear map det: $X \times X \times X \rightarrow F$ has been fixed, we can identify the exterior product with the dual space by means of the map $X \wedge X \stackrel{\approx}{\rightarrow} X^{*}$ such that $x \wedge y \mapsto \operatorname{det}(x, y,-) \in \operatorname{hom}(X, F)$. And in a dual way we can identify $X^{*} \wedge X^{*}$ with $X$ through det $^{*}$, the dual map of det. Consider three tridimensional vector spaces $X_{i}(i=1,2,3)$, and define:

$$
\mathcal{L}=\operatorname{sl}\left(X_{1}\right) \oplus \operatorname{sl}\left(X_{2}\right) \oplus \operatorname{sl}\left(X_{3}\right) \oplus X_{1} \otimes X_{2} \otimes X_{3} \oplus X_{1}^{*} \otimes X_{2}^{*} \otimes X_{3}^{*},
$$

endowed with a Lie algebra structure with the product

$$
\begin{aligned}
{\left[\otimes f_{i}, \otimes x_{i}\right] } & =\frac{1}{2} \sum_{\substack{k=1,2,3 \\
i \neq j \neq k}} f_{i}\left(x_{i}\right) f_{j}\left(x_{j}\right)\left(f_{k}(-) x_{k}-\frac{1}{3} f_{k}\left(x_{k}\right) \operatorname{id}_{X_{k}}\right) \\
{\left[\otimes x_{i}, \otimes y_{i}\right] } & =\otimes\left(x_{i} \wedge y_{i}\right) \\
{\left[\otimes f_{i}, \otimes g_{i}\right] } & =\otimes\left(f_{i} \wedge g_{i}\right)
\end{aligned}
$$

for any $x_{i}, y_{i} \in X_{i}, f_{i}, g_{i} \in X_{i}^{*}$, with the wedge products as above, and where the actions of the Lie subalgebra $\sum \operatorname{sl}\left(X_{i}\right)$ on $X_{1} \otimes X_{2} \otimes X_{3}$ and $X_{1}^{*} \otimes X_{2}^{*} \otimes X_{3}^{*}$ are the natural ones (the $i$-th simple ideal acts on the $i$-th slot). The Lie algebra $\mathcal{L}$ is isomorphic to $\mathfrak{e}_{6}$.

Following Hesselink ([24]), we say that a grading is special if and only if its 0-homogeneous component is zero. We can observe that $\mathfrak{e}_{6}$ admits a special $\mathbb{Z}_{3}^{3}$-grading with the nonzero homogeneous components of the same dimension $(78 / 26=3)$. Since $\mathcal{L}=\mathcal{L}_{\overline{0}} \oplus \mathcal{L}_{\overline{1}} \oplus \mathcal{L}_{\overline{2}}$ is a $\mathbb{Z}_{3}$-grading for $\mathcal{L}_{\overline{0}}=$ 
$\operatorname{sl}\left(X_{1}\right) \oplus \operatorname{sl}\left(X_{2}\right) \oplus \operatorname{sl}\left(X_{3}\right), \mathcal{L}_{\overline{1}}=X_{1} \otimes X_{2} \otimes X_{3}$ and $\mathcal{L}_{\overline{2}}=X_{1}^{*} \otimes X_{2}^{*} \otimes X_{3}^{*}$, take $\phi_{1}$ the automorphism which induces the grading, that is, $\left.\phi_{1}\right|_{\mathcal{L}_{\bar{i}}}=\omega^{i} \operatorname{id}_{\mathcal{L}_{\bar{i}}}$ where $\omega=e^{\frac{2 \pi I}{3}}$ is a primitive cubic root of the unit.

In order to provide the other automorphisms, take into account the following observation. If $\rho_{i}: X_{i} \rightarrow X_{i}, i=1,2,3$, are linear maps preserving det: $X_{i}^{3} \rightarrow F$ (that is, $\operatorname{det}\left(x_{i}, y_{i}, z_{i}\right)=\operatorname{det}\left(\rho_{i}\left(x_{i}\right), \rho_{i}\left(y_{i}\right), \rho_{i}\left(z_{i}\right)\right)$, or equivalently, $\left.\operatorname{det} \rho_{i}=1\right)$, the linear map $\rho_{1} \otimes \rho_{2} \otimes \rho_{3}: \mathcal{L}_{\overline{1}} \rightarrow \mathcal{L}_{\overline{1}}$ can be uniquely extended to an automorphism of $\mathcal{L}$ such that its restriction to $\operatorname{sl}\left(V_{i}\right) \subset \mathcal{L}_{\overline{0}}$ is the conjugation map $g \mapsto \rho_{i} g \rho_{i}^{-1}$.

Next fix basis $\left\{u_{0}, u_{1}, u_{2}\right\}$ of $X_{1},\left\{v_{0}, v_{1}, v_{2}\right\}$ of $X_{2}$, and $\left\{w_{0}, w_{1}, w_{2}\right\}$ of $X_{3}$ with $\operatorname{det}\left(u_{0}, u_{1}, u_{2}\right)=\operatorname{det}\left(v_{0}, v_{1}, v_{2}\right)=\operatorname{det}\left(w_{0}, w_{1}, w_{2}\right)=1$. Consider now $\phi_{2}$ the unique automorphism of $\mathfrak{e}_{6}$ extending the map

$$
u_{i} \otimes v_{j} \otimes w_{k} \mapsto u_{i+1} \otimes v_{j+1} \otimes w_{k+1}
$$

(indices module 3 ). This is of course an order three semisimple automorphism commuting with $\phi_{1}$. Finally let $\phi_{3}$ be the unique automorphism of $\mathfrak{e}_{6}$ extending the map

$$
u_{i} \otimes v_{j} \otimes w_{k} \mapsto \omega^{i} u_{i} \otimes \omega^{j} v_{j} \otimes \omega^{k} w_{k}=\omega^{i+j+k} u_{i} \otimes v_{j} \otimes w_{k} .
$$

This is also semisimple and commutes with $\phi_{1}$ and $\phi_{2}$. Thus the set $\left\{\phi_{i}\right\}_{i=1}^{3}$ induces a $\mathbb{Z}_{3}^{3}$-grading on $\mathfrak{e}_{6}$. A computation of its 0-homogeneous component will suffice to prove that this grading is nontoral. A first calculation reveals that the subalgebra of elements fixed by $\phi_{1}$ and $\phi_{2}$ is the linear span of

$$
\begin{array}{ll}
\left\{u_{1} \otimes u_{2}^{*}+u_{2} \otimes u_{3}^{*}+u_{3} \otimes u_{1}^{*},\right. & u_{1} \otimes u_{3}^{*}+u_{2} \otimes u_{1}^{*}+u_{3} \otimes u_{2}^{*}, \\
v_{1} \otimes v_{2}^{*}+v_{2} \otimes v_{3}^{*}+v_{3} \otimes v_{1}^{*}, & v_{1} \otimes v_{3}^{*}+v_{2} \otimes v_{1}^{*}+v_{3} \otimes v_{2}^{*}, \\
w_{1} \otimes w_{2}^{*}+w_{2} \otimes w_{3}^{*}+w_{3} \otimes w_{1}^{*}, & \left.w_{1} \otimes w_{3}^{*}+w_{2} \otimes w_{1}^{*}+w_{3} \otimes w_{2}^{*}\right\},
\end{array}
$$

where we have taken $u_{i}^{*}=u_{i+1} \wedge u_{i+2}, v_{i}^{*}=v_{i+1} \wedge v_{i+2}$ and $w_{i}^{*}=w_{i+1} \wedge w_{i+2}$ the dual basis of $X_{1}^{*}, X_{2}^{*}$ and $X_{3}^{*}$ respectively. While the corresponding fixed subalgebra for $\phi_{1}$ and $\phi_{3}$ is the linear span of

$$
\begin{array}{ll}
\left\{u_{1} \otimes u_{1}^{*}-u_{2} \otimes u_{2}^{*},\right. & u_{1} \otimes u_{1}^{*}-u_{3} \otimes u_{3}^{*}, \\
v_{1} \otimes v_{1}^{*}-v_{2} \otimes v_{2}^{*}, & v_{1} \otimes v_{1}^{*}-v_{3} \otimes v_{3}^{*}, \\
w_{1} \otimes w_{1}^{*}-w_{2} \otimes w_{2}^{*}, & \left.w_{1} \otimes w_{1}^{*}-w_{3} \otimes w_{3}^{*}\right\},
\end{array}
$$

again a six-dimensional abelian subalgebra. The intersection of both Cartan subalgebras is obviously zero and therefore our grading is special and nontoral. Similar computations prove that the rest of the homogeneous components are three-dimensional. The grading produced by any of the automorphisms $\phi_{i}$ is of type $(24,27)$, since they are in the same conjugacy class. The grading produced by any of the three couples $\left\{\phi_{i}, \phi_{j}\right\}$ has one 
six-dimensional component and eight nine-dimensional ones. The grading induced by the three automorphisms together is of type $(0,0,26)$. Now we must go down to see this grading in $\mathfrak{f}_{4}$.

To a certain extent, the nice 3 -symmetry described in $\mathfrak{e}_{6}$ is inherited by $\mathfrak{f}_{4}$. Indeed graphically speaking, $\mathfrak{f}_{4}$ arises by folding $\mathfrak{e}_{6}$. More precisely, taking $X_{2}=X_{3}$ we can consider on $\mathfrak{e}_{6}$ the unique automorphism $\tau: \mathfrak{e}_{6} \rightarrow \mathfrak{e}_{6}$ extension of $u \otimes v \otimes w \mapsto u \otimes w \otimes v$. This is an order two automorphism commuting with the previous $\phi_{i}$ for $i=1,2,3$. The subalgebra of elements fixed by $\tau$ is

$$
\operatorname{sl}\left(X_{1}\right) \oplus \operatorname{sl}\left(X_{2}\right) \oplus X_{1} \otimes \operatorname{Sym}^{2}\left(X_{2}\right) \oplus X_{1}^{*} \otimes \operatorname{Sym}^{2}\left(X_{2}^{*}\right),
$$

where $\operatorname{Sym}^{n} X_{i}$ denotes the symmetric powers (as in [17, p. 473]). This is a simple Lie algebra of dimension 52 , hence $\mathfrak{f}_{4}$. Furthermore, denoting also by $\phi_{i}: \mathfrak{f}_{4} \rightarrow \mathfrak{f}_{4}$ the restriction of the corresponding automorphisms of $\mathfrak{e}_{6}$ to fix $\tau$, the set $\left\{\phi_{i}\right\}_{i=1}^{3}$ is also a set of commuting semisimple order three automorphisms of $\mathfrak{f}_{4}$ with no fixed points other than 0 . So it induces a special nontoral $\mathbb{Z}_{3}^{3}$-grading on $\mathfrak{f}_{4}$ with all its homogeneous components of the same dimension $(52 / 26=2)$, of type $(0,26)$.

\section{2. $\mathbb{Z}_{2}^{5}$-grading on $\mathfrak{f}_{4}$}

The group of automorphisms inducing this grading is also an elementary p-group so that it is described in Griess work [19, Th. 7.3, p. 277]. Moreover, the grading is pure (that is, there is some homogeneous component which contains a Cartan subalgebra), therefore it also appears in Hesselink's paper [24, Table1, p. 146]. As before an instantaneous computer calculation provides its type, $(24,0,0,7)$, as well as the description of its homogeneous components in terms of the fixed basis:

$$
\begin{aligned}
& L_{0,0,0,0,0}=0, \\
& L_{0,0,0,0,1}=\left\langle b_{2}+b_{26}\right\rangle, \\
& L_{0,0,0,1,0}=\left\langle b_{41}-b_{17}\right\rangle, \\
& L_{0,0,0,1,1}=\left\langle b_{43}-b_{19}\right\rangle, \\
& L_{0,0,1,0,0}=\left\langle b_{38}-b_{14}\right\rangle, \\
& L_{0,0,1,0,1}=\left\langle b_{35}-b_{11}\right\rangle, \\
& L_{0,0,1,1,0}=\left\langle b_{3}+b_{27}, b_{34}-b_{10}, b_{39}-b_{15}, b_{48}-b_{24}\right\rangle, \\
& L_{0,0,1,1,1}=\left\langle b_{30}-b_{6}\right\rangle, \\
& L_{0,1,0,0,0}=\left\langle b_{1}+b_{25}\right\rangle, \\
& L_{0,1,0,0,1}=\left\langle b_{5}+b_{29}\right\rangle, \\
& L_{0,1,0,1,0}=\left\langle b_{28}-b_{4}, b_{40}-b_{16}, b_{44}-b_{20}, b_{22}+b_{46}\right\rangle, \\
& L_{0,1,0,1,1}=\left\langle b_{45}-b_{21}\right\rangle,
\end{aligned}
$$




$$
\begin{aligned}
& L_{0,1,1,0,0}=\left\langle b_{7}+b_{31}, b_{37}-b_{13}, b_{42}-b_{18}, b_{47}-b_{23}\right\rangle, \\
& L_{0,1,1,0,1}=\left\langle b_{32}-b_{8}\right\rangle, \\
& L_{0,1,1,1,0}=\left\langle b_{36}-b_{12}\right\rangle, \\
& L_{0,1,1,1,1}=\left\langle b_{33}-b_{9}\right\rangle, \\
& L_{1,0,0,0,0}=\left\langle t_{\alpha_{1}}, t_{\alpha_{2}}, t_{\alpha_{3}}, t_{\alpha_{4}}\right\rangle, \\
& L_{1,0,0,0,1}=\left\langle b_{26}-b_{2}\right\rangle, \\
& L_{1,0,0,1,0}=\left\langle b_{17}+b_{41}\right\rangle, \\
& L_{1,0,0,1,1}=\left\langle b_{19}+b_{43}\right\rangle, \\
& L_{1,0,1,0,0}=\left\langle b_{14}+b_{38}\right\rangle, \\
& L_{1,0,1,0,1}=\left\langle b_{11}+b_{35}\right\rangle, \\
& L_{1,0,1,1,0}=\left\langle b_{27}-b_{3}, b_{10}+b_{34}, b_{15}+b_{39}, b_{24}+b_{48}\right\rangle, \\
& L_{1,0,1,1,1}=\left\langle b_{6}+b_{30}\right\rangle, \\
& L_{1,1,0,0,0}=\left\langle b_{25}-b_{1}\right\rangle, \\
& L_{1,1,0,0,1}=\left\langle b_{29}-b_{5}\right\rangle, \\
& L_{1,1,0,1,0}=\left\langle b_{4}+b_{28}, b_{16}+b_{40}, b_{20}+b_{44}, b_{46}-b_{22}\right\rangle, \\
& L_{1,1,0,1,1}=\left\langle b_{21}+b_{45}\right\rangle, \\
& L_{1,1,1,0,0}=\left\langle b_{31}-b_{7}, b_{13}+b_{37}, b_{18}+b_{42}, b_{23}+b_{47}\right\rangle, \\
& L_{1,1,1,0,1}=\left\langle b_{8}+b_{32}\right\rangle, \\
& L_{1,1,1,1,0}=\left\langle b_{12}+b_{36}\right\rangle, \\
& L_{1,1,1,1,1}=\left\langle b_{9}+b_{33}\right\rangle .
\end{aligned}
$$

But any of the standard models of $\mathfrak{f}_{4}$ would also do for describing the grading in a basis-free manner. One of the best known models is that of Schafer ([39, p. 112]) as derivations of the Albert algebra. Starting from the nontoral $\mathbb{Z}_{2}^{3}$ grading on the Cayley algebra $C=\oplus_{g \in \mathbb{Z}_{2}^{3}} C_{g}$ we obtained the $\mathbb{Z}_{2}^{5}$-grading on the Albert algebra $J$ given by

$$
\begin{array}{lll}
J_{e, 0,0}=<E_{1}, E_{2}, E_{3}>, & J_{g, 0,0}=0(g \neq e), & \\
J_{g, 0,1}=C_{g}^{(1)}, & J_{g, 1,1}=C_{g}^{(2)}, & J_{g, 1,0}=C_{g}^{(3)},
\end{array}
$$

with $g, e=(0,0,0) \in \mathbb{Z}_{2}^{3}$. Obviously the grading induced in $L=\operatorname{Der}(J)=$ $\left[R_{J}, R_{J}\right]$ has as homogeneous components $L_{a}=\left\{d \in \operatorname{Der}(J) \mid d\left(J_{b}\right) \subset\right.$ $\left.J_{a+b} \forall b \in \mathbb{Z}_{2}^{5}\right\}$, therefore $L_{e, 0,0}=0$ and

$$
\begin{aligned}
& L_{g, 0,1}=\left\{\left[R_{x^{(1)}}, R_{E_{2}-E_{3}}\right] \mid x \in C_{g}\right\} \\
& L_{g, 1,1}=\left\{\left[R_{x^{(2)}}, R_{E_{3}-E_{1}}\right] \mid x \in C_{g}\right\} \\
& L_{g, 1,0}=\left\{\left[R_{x^{(3)}}, R_{E_{1}-E_{2}}\right] \mid x \in C_{g}\right\} \quad \forall g \in \mathbb{Z}_{2}^{3} \\
& L_{g, 0,0}=\left\{D_{U} \mid U \in N_{g}\right\} \oplus\left\{D_{r_{x}} \mid x \in\left(C_{0}\right)_{g}\right\} \oplus\left\{D_{l_{x}} \mid x \in\left(C_{0}\right)_{g}\right\} \quad \forall e \neq g \in \mathbb{Z}_{2}^{3}
\end{aligned}
$$

where

- $N_{g}=\left\{d \in \operatorname{Der}(C) \mid d\left(C_{b}\right) \subset C_{g+b} \forall b \in \mathbb{Z}_{2}^{3}\right\}$ are the components of the grading induced on $\mathfrak{g}_{2}$, all of them two-dimensional and Cartan subalgebras, except for $N_{e}=0$, 
- $r_{x}$ and $l_{x}$ are the right and left multiplication operators on $C$,

- if $U \in \mathrm{o}(C, n), D_{U} \in \operatorname{Der}(J)$ is the derivation given by

$$
E_{i} \mapsto 0, x^{(1)} \mapsto \overline{U(\bar{x})}^{(1)}, x^{(2)} \mapsto U^{\prime}(x)^{(2)}, x^{(3)} \mapsto U^{\prime \prime}(x)^{(3)},
$$

where $U^{\prime}$ and $U^{\prime \prime}$ are the elements in o $(C, n)$ given by the local triality principle [39, p. 88], that is, $U(x y)=U^{\prime}(x) y+x U^{\prime \prime}(y)$ for all $x, y \in C$. Then clearly $h_{4}=7\left(\operatorname{dim} L_{g, 0,0}=2+1+1=4\right)$, also $h_{1}=8 \cdot 3=24$ and the grading is of type $(24,0,0,7)$.

Anyway, we think that there is a more intuitive way of looking at this grading, as well as at the gradings obtained by crossing gradings on the Cayley algebra $C$ with gradings on $H_{3}(F)$. Recall from Subsection 3.3 that if $H \equiv H_{3}(F)=\left\{x \in M_{3}(F) \mid x=x^{t}\right\}$ and $K \equiv K_{3}(F)=\left\{x \in M_{3}(F) \mid\right.$ $\left.x=-x^{t}\right\}$, we could write

$$
J=H \oplus K \otimes C_{0} .
$$

Since $\mathfrak{f}_{4}=\operatorname{Der}(J)$, there must exist some model of $\mathfrak{f}_{4}$ in these terms. In fact we can see $\mathfrak{f}_{4}$ as

$$
L=\operatorname{Der}(C) \oplus K \oplus H_{0} \otimes C_{0}
$$

identifying the Lie algebra $K$ (subalgebra of $M_{3}(F)^{-}$) to $\operatorname{Der}\left(H_{3}(F)\right.$ ) in the known Tits unified construction for the Lie exceptional algebras (for instance, see [39, p. 122]).

Consider a $G_{1}$-grading on the Jordan algebra $H=\oplus_{g \in G_{1}} H_{g}$. This grading will come from a grading on $M_{3}(F)$ so that the Lie algebra $K$ will also have an induced grading. Take now the $\mathbb{Z}_{2}^{3}$-grading on the Cayley algebra $C=\oplus_{g \in G_{2}=\mathbb{Z}_{2}^{3}} C_{g}$ and the induced grading $\operatorname{Der}(C)=\oplus_{g \in G_{2}} N_{g}$. All this material induces a $G_{1} \times G_{2}$-grading on $J$ and also on $L$ given by

$$
\begin{array}{ll}
J_{g_{1}, e}=H_{g_{1}}, & J_{g_{1}, g_{2}}=K_{g_{1}} \otimes\left(C_{0}\right)_{g_{2}}, \\
L_{g_{1}, e}=K_{g_{1}}, & L_{e, g_{2}}=N_{g_{2}} \oplus\left(H_{0}\right)_{e} \otimes\left(C_{0}\right)_{g_{2}}, \quad L_{g_{1}, g_{2}}=\left(H_{0}\right)_{g_{1}} \otimes\left(C_{0}\right)_{g_{2}} .
\end{array}
$$

In the case of the $\mathbb{Z}_{2}^{5}$-grading we have $G_{1}=\mathbb{Z}_{2}^{2}$, with the gradings on $H$ and $K$ given by

$$
\begin{array}{llll}
H_{0,0}=\left\langle E_{1}, E_{2}, E_{3}\right\rangle & H_{0,1}=\left\langle e_{12}+e_{21}\right\rangle & H_{1,1}=\left\langle e_{23}+e_{32}\right\rangle & H_{1,0}=\left\langle e_{13}+e_{31}\right\rangle \\
K_{0,0}=0 & K_{0,1}=\left\langle e_{12}-e_{21}\right\rangle & K_{1,1}=\left\langle e_{23}-e_{32}\right\rangle & K_{1,0}=\left\langle e_{13}-e_{31}\right\rangle
\end{array}
$$

and $\operatorname{dim}\left(C_{0}\right)_{g}=1, \operatorname{dim} N_{g}=2$ for all $g \in \mathbb{Z}_{2}^{3} \backslash\{(0,0,0)\}$. Therefore

$$
\begin{array}{ll}
\operatorname{dim} J_{e, e}=\operatorname{dim} H_{e}=3 & \operatorname{dim} L_{e, e}=0 \\
\operatorname{dim} J_{e, g_{2}}=0 & \operatorname{dim} L_{e, g_{2}}=\operatorname{dim}\left(N_{g_{2}}+\left(H_{0}\right)_{e} \otimes\left(C_{0}\right)_{g_{2}}\right)=4 \\
\operatorname{dim} J_{g_{1}, e}=\operatorname{dim} H_{g_{1}}=1 & \operatorname{dim} L_{g_{1}, e}=\operatorname{dim} K_{g_{1}}=1 \\
\operatorname{dim} J_{g_{1}, g_{2}}=\operatorname{dim} K_{g_{1}} \otimes\left(C_{0}\right)_{g_{2}}=1 & \operatorname{dim} L_{g_{1}, g_{2}}=\operatorname{dim}\left(H_{0}\right)_{g_{1}} \otimes\left(C_{0}\right)_{g_{2}}=1
\end{array}
$$

and so the grading is of type $(24,0,1)$ on $J$, and $(24,0,0,7)$ on $L=\mathfrak{f}_{4}$, as we knew from previous sections. 


\section{3. $\mathbb{Z}_{2}^{3} \times \mathbb{Z}$-grading of $\mathfrak{f}_{4}$}

By contrast with previous cases, as long as we know this grading does not appear in the mathematical literature. Again a simple computer aided calculation reveals that the fine $\mathbb{Z}_{2}^{3} \times \mathbb{Z}$-grading of type $(31,0,7)$ is

$$
\begin{aligned}
& L_{0,0,0,-2}=0, \quad L_{0,0,0,-1}=\left\langle b_{2}+b_{9}\right\rangle, \\
& L_{0,0,0,0}=\left\langle t_{\alpha_{2}}+2 t_{\alpha_{3}}+t_{\alpha_{4}}\right\rangle, \quad L_{0,0,0,1}=\left\langle b_{33}-b_{26}\right\rangle, \\
& L_{0,0,0,2}=0, \quad L_{0,0,1,-2}=\left\langle b_{22}+b_{28}\right\rangle, \\
& L_{0,0,1,-1}=\left\langle b_{35}-b_{21}\right\rangle, \quad L_{0,0,1,1}=\left\langle b_{45}-b_{11}\right\rangle \text {, } \\
& L_{0,0,1,2}=\left\langle b_{46}-b_{4}\right\rangle, \quad L_{0,1,0,-2}=\left\langle b_{15}-b_{10}\right\rangle, \\
& L_{0,1,0,-1}=\left\langle b_{6}-b_{5}\right\rangle, \quad L_{0,1,0,1}=\left\langle b_{29}+b_{30}\right\rangle \text {, } \\
& L_{0,1,0,2}=\left\langle b_{39}-b_{34}\right\rangle, \quad L_{0,1,1,-2}=\left\langle b_{23}+b_{31}\right\rangle, \\
& L_{0,1,1,-1}=\left\langle b_{19}+b_{32}\right\rangle, \quad L_{0,1,1,1}=\left\langle b_{8}+b_{43}\right\rangle \text {, } \\
& L_{0,1,1,2}=\left\langle b_{47}-b_{7}\right\rangle, \quad L_{1,0,0,-2}=\left\langle b_{12}\right\rangle, \\
& L_{1,0,0,-1}=\left\langle b_{9}-b_{2}\right\rangle, \quad L_{1,0,0,1}=\left\langle b_{26}+b_{33}\right\rangle \text {, } \\
& L_{1,0,0,2}=\left\langle b_{36}\right\rangle, \quad L_{1,0,1,-2}=\left\langle b_{28}-b_{22}\right\rangle, \\
& L_{1,0,1,-1}=\left\langle b_{21}+b_{35}\right\rangle, \quad L_{1,0,1,1}=\left\langle b_{11}+b_{45}\right\rangle, \\
& L_{1,0,1,2}=\left\langle b_{4}+b_{46}\right\rangle, \quad L_{1,1,0,-2}=\left\langle b_{10}+b_{15}\right\rangle, \\
& L_{1,1,0,-1}=\left\langle b_{5}+b_{6}\right\rangle, \quad L_{1,1,0,1}=\left\langle b_{30}-b_{29}\right\rangle, \\
& L_{1,1,0,2}=\left\langle b_{34}+b_{39}\right\rangle, \quad L_{1,1,1,-2}=\left\langle b_{31}-b_{23}\right\rangle, \\
& L_{1,1,1,-1}=\left\langle b_{32}-b_{19}\right\rangle, \quad L_{1,1,1,1}=\left\langle b_{43}-b_{8}\right\rangle \text {, } \\
& L_{1,1,1,2}=\left\langle b_{7}+b_{47}\right\rangle, \quad L_{1,0,0,0}=\left\langle t_{\alpha_{2}}, \frac{t_{\alpha_{1}}}{2}+t_{\alpha_{3}}, t_{\alpha_{4}}\right\rangle \text {, } \\
& L_{0,0,1,0}=\left\langle b_{38}-b_{14}, b_{40}-b_{16}, b_{44}-b_{20}\right\rangle, \\
& L_{0,1,0,0}=\left\langle b_{1}+b_{25}, b_{3}+b_{27}, b_{48}-b_{24}\right\rangle \text {, } \\
& L_{0,1,1,0}=\left\langle b_{37}-b_{13}, b_{41}-b_{17}, b_{42}-b_{18}\right\rangle \text {, } \\
& L_{1,0,1,0}=\left\langle b_{14}+b_{38}, b_{16}+b_{40}, b_{20}+b_{44}\right\rangle \text {, } \\
& L_{1,1,0,0}=\left\langle b_{25}-b_{1}, b_{27}-b_{3}, b_{24}+b_{48}\right\rangle, \\
& L_{1,1,1,0}=\left\langle b_{13}+b_{37}, b_{17}+b_{41}, b_{18}+b_{42}\right\rangle \text {. }
\end{aligned}
$$

But we can detect this grading without reference to explicit computations using again the model $\mathfrak{f}_{4}=L=\operatorname{Der}(C) \oplus K \oplus H_{0} \otimes C_{0}$. We can write the $\mathbb{Z}$-grading on $H$ given in (3.2), jointly with the induced one in $K$ by its extension to $M_{3}(F)$, in the following equivalent form (using a suitable involution $x \mapsto p x^{t} p^{-1}$ instead of the transposition):

$$
\begin{array}{ll}
H_{2}=\left\langle e_{23}\right\rangle, & K_{2}=0, \\
H_{1}=\left\langle e_{13}+e_{21}\right\rangle, & K_{1}=\left\langle e_{13}-e_{21}\right\rangle, \\
H_{e}=\left\langle E_{1}, E_{2}+E_{3}\right\rangle, & K_{e}=\left\langle E_{2}-E_{3}\right\rangle, \\
H_{-1}=\left\langle e_{12}+e_{31}\right\rangle, & K_{-1}=\left\langle e_{12}-e_{31}\right\rangle, \\
H_{-2}=\left\langle e_{32}\right\rangle, & K_{-2}=0 .
\end{array}
$$


Thus, by crossing it with the $\mathbb{Z}_{2}^{3}$-grading on $C$ we obtain

$$
\begin{array}{ll}
\operatorname{dim} J_{2, e}=\operatorname{dim} H_{2}=1 & \operatorname{dim} L_{2, e}=0 \\
\operatorname{dim} J_{1, e}=\operatorname{dim} H_{1}=1 & \operatorname{dim} L_{1, e}=\operatorname{dim} K_{1}=1 \\
\operatorname{dim} J_{e, e}=\operatorname{dim} H_{e}=2 & \operatorname{dim} L_{e, e}=\operatorname{dim} K_{e}=1 \\
\operatorname{dim} J_{2, g}=0 & \operatorname{dim} L_{2, g}=\operatorname{dim} H_{2} \otimes\left(C_{0}\right)_{g}=1 \\
\operatorname{dim} J_{1, g}=\operatorname{dim} K_{1} \otimes\left(C_{0}\right)_{g}=1 & \operatorname{dim} L_{1, g}=\operatorname{dim} H_{1} \otimes\left(C_{0}\right)_{g}=1 \\
\operatorname{dim} J_{e, g}=\operatorname{dim} K_{e} \otimes\left(C_{0}\right)_{g}=1 & \operatorname{dim} L_{e, g}=\operatorname{dim} N_{g} \oplus\left(H_{0}\right)_{e} \otimes\left(C_{0}\right)_{g}=3
\end{array}
$$

and the same dimensions of the homogeneous components with opposite indices. So the grading is of type $(25,1)$ on $J$ and $(31,0,7)$ on $\mathfrak{f}_{4}$.

\subsection{The remaining nontoral gradings}

Any of the detected gradings on $\mathfrak{f}_{4}$ can be obtained by projections given by epimorphisms from the universal grading groups corresponding to the fine gradings. However, it is worth to point out that all of them can also be described by using different models of the algebra. More precisely, consider a $G$-grading on a simple Lie algebra $L$ without outer automorphisms (equivalently, without automorphisms of the Dynkin diagram). Then $G$ is a product of cyclic groups $G_{i}$, each of which produces certain cyclic $G_{i}$-grading. The zero component of most of these $G_{i}$-gradings is a direct sum of Lie subalgebras of type either $\operatorname{sl}(V)$ or $\operatorname{so}(V)$. As modules for the zero component, the remaining components are isomorphic to either tensor of natural modules or spin ones, respectively (see [12] for more details). In the first case, it is possible to describe the $G$-grading in a similar way that just illustrated with the $\mathbb{Z}_{3}^{3}$-grading on $\mathfrak{f}_{4}$. We now give some sketches of how this basis-free method works for the other gradings found in $\mathfrak{f}_{4}$, for instance the $\mathbb{Z}_{2}^{2} \times \mathbb{Z}_{4}$ and $\mathbb{Z}_{2}^{2} \times \mathbb{Z}_{8}$-gradings.

Let $V$ and $W$ be $F$-vector spaces of dimensions 2 and 4 respectively. According to [12], $\mathfrak{f}_{4}$ can be seen in the way

$$
\mathcal{L}=\operatorname{sl}(V) \oplus \operatorname{sl}(W) \oplus V \otimes W \oplus \operatorname{Sym}^{2} V \otimes \bigwedge^{2} W \oplus V \otimes \bigwedge^{3} W
$$

and its product given in a way similar to (7.1).

One of the advantages of this model is that given linear maps $\varphi: V \rightarrow V$ and $\tilde{\varphi}: W \rightarrow W$ such that the first one preserves det: $\bigwedge^{2} V \rightarrow F$ and the second preserves det: $\bigwedge^{4} W \rightarrow F$, the map $\varphi \otimes \tilde{\varphi}: V \otimes W \rightarrow V \otimes W$ can be uniquely extended to an automorphism of the algebra $\mathcal{L} \cong \mathfrak{f}_{4}$, in such a way that the restriction of this automorphism to $\operatorname{sl}(V)$ is the map $g \mapsto \varphi g \varphi^{-1}$, and to $\operatorname{sl}(W)$ is the map $g \mapsto \tilde{\varphi} g \tilde{\varphi}^{-1}$. Now, denote by

$$
\mathcal{L}_{\overline{0}}=\operatorname{sl}(V) \oplus \operatorname{sl}(W), \quad \mathcal{L}_{\overline{1}}=V \otimes W, \quad \mathcal{L}_{\overline{2}}=\operatorname{Sym}^{2} V \otimes \bigwedge^{2} W, \quad \mathcal{L}_{\overline{3}}=V \otimes \bigwedge^{3} W .
$$


As $\mathcal{L}=\mathcal{L}_{\overline{0}} \oplus \mathcal{L}_{\overline{1}} \oplus \mathcal{L}_{\overline{2}} \oplus \mathcal{L}_{\overline{3}}$ is a $\mathbb{Z}_{4}$-grading, take $\phi_{1}$ the automorphism which gives the grading, that is, $\left.\phi_{1}\right|_{\mathcal{L}_{\bar{i}}}=I^{i} \mathrm{id}_{\mathcal{L}_{\bar{i}}}$ where $I$ is a primitive fourth root of the unit. Let us fix $\left\{u_{0}, u_{1}\right\}$ a basis of $V$ with $\operatorname{det}\left(u_{0}, u_{1}\right)=1$, and $\left\{w_{0}, w_{1}, w_{2}, w_{3}\right\}$ a basis of $W$ with $\operatorname{det}\left(w_{0}, w_{1}, w_{2}, w_{3}\right)=1$. Take $\varphi_{2}: V \rightarrow$ $V, u_{0} \mapsto I u_{0}, u_{1} \mapsto-I u_{1}$ and $\tilde{\varphi}_{2}: W \rightarrow W, w_{0} \mapsto w_{0}, w_{1} \mapsto w_{1}, w_{2} \mapsto$ $-w_{2}, w_{3} \mapsto-w_{3}$. Define $\phi_{2} \in \mathfrak{F}_{4}$ as the extension of $\varphi_{2} \otimes \tilde{\varphi}_{2}$. Take now $\varphi_{3}: V \rightarrow V, u_{0} \mapsto u_{1}, u_{1} \mapsto-u_{0}$ and $\tilde{\varphi}_{3}: W \rightarrow W, w_{0} \mapsto w_{2}, w_{1} \mapsto w_{3}, w_{2} \mapsto$ $w_{0}, w_{3} \mapsto w_{1}$. Define then $\phi_{3} \in \mathfrak{F}_{4}$ as the extension of $\varphi_{3} \otimes \tilde{\varphi}_{3}$. Take finally $E$ a primitive eighth root of the unit, and define $\tilde{\varphi}_{4}: W \rightarrow W$ by $\tilde{\varphi}_{4}\left(w_{0}\right)=E^{5} w_{2}, \tilde{\varphi}_{4}\left(w_{1}\right)=E^{3} w_{3}, \tilde{\varphi}_{4}\left(w_{2}\right)=E^{5} w_{0}, \tilde{\varphi}_{4}\left(w_{3}\right)=E^{3} w_{1}$. Consider $\phi_{4} \in \mathfrak{F}_{4}$ the extension of $\varphi_{3} \otimes \tilde{\varphi}_{4}$. The set $\left\{\phi_{i}\right\}_{i=1}^{4}$ is a commutative set of semisimple automorphisms and one can see with some easy though boring hand computations that the grading induced by $\left\{\phi_{1}, \phi_{2}, \phi_{3}\right\}$ is a $\mathbb{Z}_{2}^{2} \times \mathbb{Z}_{4^{-}}$ grading of type $(0,8,2,0,6)$, the grading produced by $\left\{\phi_{1}, \phi_{2}, \phi_{4}\right\}$ is a $\mathbb{Z}_{2}^{2} \times$ $\mathbb{Z}_{8}$-grading of type $(19,6,7)$ and the grading produced by $\left\{\phi_{1}, \phi_{2}, \phi_{3}, \phi_{4}\right\}$ is a $\mathbb{Z}_{2}^{3} \times \mathbb{Z}_{8}$-grading of type $(31,0,7)$, equivalent to the fine $\mathbb{Z}_{2}^{3} \times \mathbb{Z}$-grading. We display their homogeneous components.

The $\mathbb{Z}_{2}^{2} \times \mathbb{Z}_{4}$-grading induced by $\left\{\phi_{1}, \phi_{2}, \phi_{3}\right\}$ is:

$$
\mathcal{L}_{\overline{0}}=\left\{\begin{array}{lll}
L_{1,1,1} & =\operatorname{diag}(a, a)_{W} & \operatorname{dim} 3 \\
L_{1,1,-1}=\operatorname{diag}(1,-1)_{V} \oplus \operatorname{diag}(b,-b)_{W} & \operatorname{dim} 5 \\
L_{1,-1,1}=\operatorname{antidiag}(1,-1)_{V} \oplus \operatorname{antidiag}(b, b)_{W} & \operatorname{dim} 5 \\
L_{1,-1,-1}=\operatorname{antidiag}(1,1)_{V} \oplus \operatorname{antidiag}(b,-b)_{W} & \operatorname{dim} 5
\end{array}\right.
$$

with $a \in \operatorname{sl}(2)$ and $b \in \operatorname{gl}(2)$,

$$
\begin{aligned}
\mathcal{L}_{\overline{1}}=\left\{\begin{array}{rlr}
L_{I, I, I}=\left\langle u_{0} \otimes w_{0}-I u_{1} \otimes w_{2}, u_{0} \otimes w_{1}-I u_{1} \otimes w_{3}\right\rangle & \operatorname{dim} 2 \\
L_{I, I,-I}=\left\langle u_{0} \otimes w_{0}+I u_{1} \otimes w_{2}, u_{0} \otimes w_{1}+I u_{1} \otimes w_{3}\right\rangle & \operatorname{dim} 2 \\
L_{I,-I, I}=\left\langle u_{0} \otimes w_{2}-I u_{1} \otimes w_{0}, u_{0} \otimes w_{3}-I u_{1} \otimes w_{1}\right\rangle & \operatorname{dim} 2 \\
L_{I,-I,-I}=\left\langle u_{0} \otimes w_{2}+I u_{1} \otimes w_{0}, u_{0} \otimes w_{3}+I u_{1} \otimes w_{1}\right\rangle & \operatorname{dim} 2
\end{array}\right. \\
\mathcal{L}_{\overline{2}}=\left\{\begin{array}{rlr}
L_{-1,1,1}= & \left\langle\left(u_{0} \cdot u_{0}+u_{1} \cdot u_{1}\right) \otimes\left(w_{0} \wedge w_{3}-w_{1} \wedge w_{2}\right),\right. & \\
& \left(u_{0} \cdot u_{0}-u_{1} \cdot u_{1}\right) \otimes\left\langle w_{0} \wedge w_{3}+w_{1} \wedge w_{2}, w_{0} \wedge w_{2}, w_{1} \wedge w_{3}\right\rangle, \\
& \left.u_{0} \cdot u_{1} \otimes\left(w_{0} \wedge w_{1}-w_{2} \wedge w_{3}\right)\right\rangle & \operatorname{dim} 5 \\
L_{-1,1,-1}= & \left\langle\left(u_{0} \cdot u_{0}+u_{1} \cdot u_{1}\right) \otimes\left(w_{0} \wedge w_{3}+w_{1} \wedge w_{2}, w_{0} \wedge w_{2}, w_{1} \wedge w_{3}\right),\right. & \\
& \left(u_{0} \cdot u_{0}-u_{1} \cdot u_{1}\right) \otimes\left(w_{0} \wedge w_{3}-w_{1} \wedge w_{2}\right), & \operatorname{dim} 5 \\
& \left.u_{0} \cdot u_{1} \otimes\left(w_{0} \wedge w_{1}+w_{2} \wedge w_{3}\right)\right\rangle & \\
L_{-1,-1,1}= & \left\langle\left(u_{0} \cdot u_{0}+u_{1} \cdot u_{1}\right) \otimes\left(w_{0} \wedge w_{1}+w_{2} \wedge w_{3}\right),\right. & \\
& \left(u_{0} \cdot u_{0}-u_{1} \cdot u_{1}\right) \otimes\left(w_{0} \wedge w_{1}-w_{2} \wedge w_{3}\right), & \\
& \left.u_{0} \cdot u_{1} \otimes\left\langle w_{0} \wedge w_{3}+w_{1} \wedge w_{2},, w_{0} \wedge w_{2}, w_{1} \wedge w_{3}\right\rangle\right\rangle & \operatorname{dim} 5 \\
L_{-1,-1,-1}= & \left\langle\left(u_{0} \cdot u_{0}+u_{1} \cdot u_{1}\right) \otimes\left(w_{0} \wedge w_{1}-w_{2} \wedge w_{3}\right),\right. & \\
& \left(u_{0} \cdot u_{0}-u_{1} \cdot u_{1}\right) \otimes\left(w_{0} \wedge w_{1}+w_{2} \wedge w_{3}\right), & \operatorname{dim} 3 \\
& \left.u_{0} \cdot u_{1} \otimes\left(w_{0} \wedge w_{3}-w_{1} \wedge w_{2}\right)\right\rangle &
\end{array}\right.
\end{aligned}
$$


and $\mathcal{L}_{\overline{3}}$ dual to $\mathcal{L}_{\overline{1}}$, hence $h_{5}=6, h_{3}=2$ and $h_{6}=8$, as we wondered. The notation antidiag $\left(x_{1}, \ldots, x_{n}\right)$ stands for the $n \times n$ matrix $\left(a_{i j}\right)$ with all entries zero except for $a_{i, n-i+1}=x_{i}$. The subindices in $L_{i j k}$ indicate that $\phi_{1}$, $\phi_{2}, \phi_{3}$ act with eigenvalues $i, j, k$ respectively. Notice that, although $\phi_{i}$ has order 4 for $i=1,2,3, \phi_{1} \phi_{2}$ and $\phi_{1} \phi_{3}$ have order 2 .

The $\mathbb{Z}_{2}^{2} \times \mathbb{Z}_{8}$-grading induced by $\left\{\phi_{1}, \phi_{4}, \phi_{2}\right\}$ is:

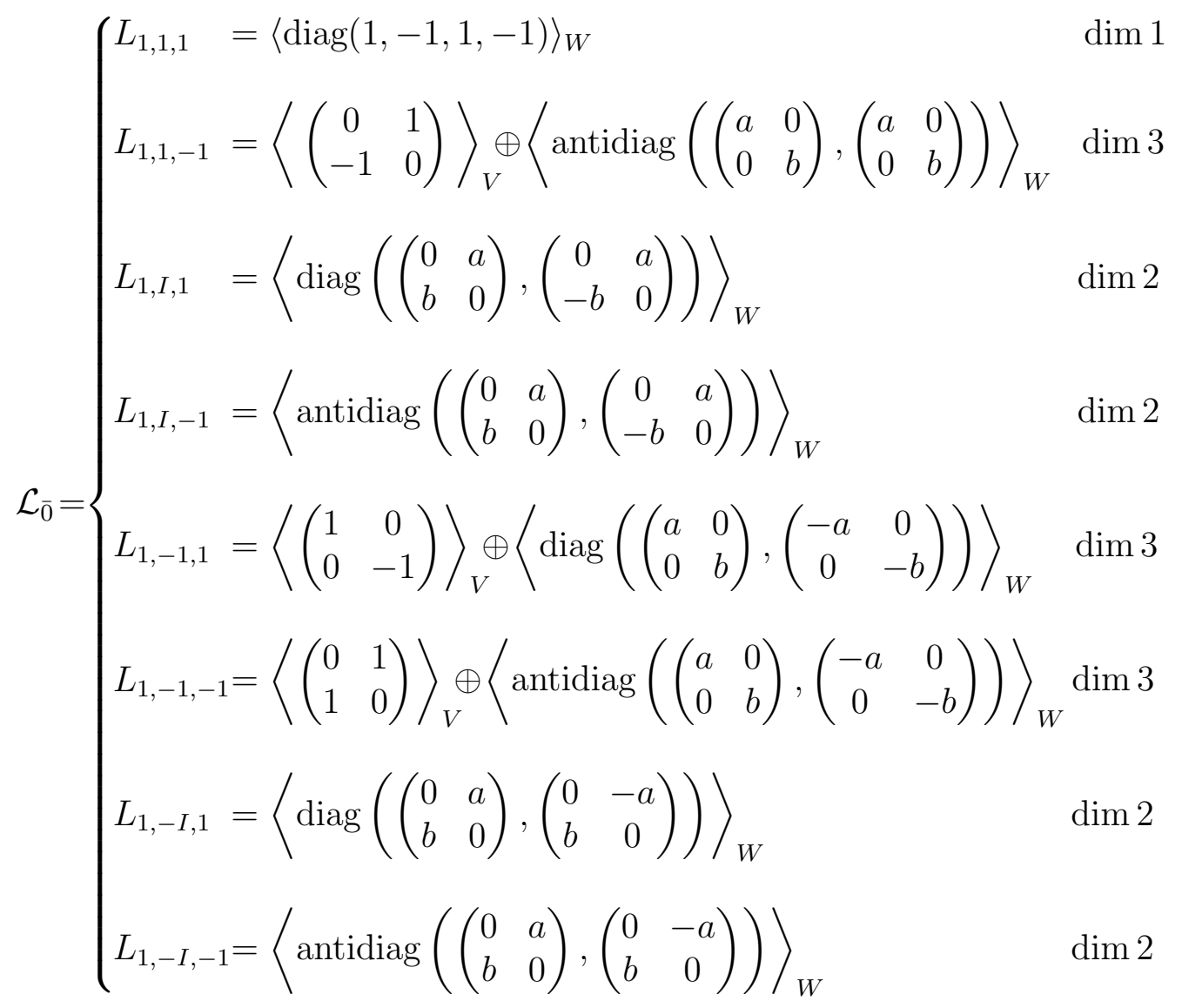

with $a, b \in F$,

$$
\mathcal{L}_{\overline{1}}=\left\{\begin{array}{llr}
L_{I, E^{7}, I}=\left\langle u_{0} \otimes w_{0}-I u_{1} \otimes w_{2}\right\rangle & \operatorname{dim} 1 \\
L_{I, E^{7},-I}=\left\langle u_{0} \otimes w_{2}-I u_{1} \otimes w_{0}\right\rangle & \operatorname{dim} 1 \\
L_{I, E^{3}, I}=\left\langle u_{0} \otimes w_{0}+I u_{1} \otimes w_{2}\right\rangle & \operatorname{dim} 1 \\
L_{I, E^{3},-I}=\left\langle u_{0} \otimes w_{2}+I u_{1} \otimes w_{0}\right\rangle & \operatorname{dim} 1 \\
L_{I, E^{5}, I}=\left\langle u_{0} \otimes w_{1}-I u_{1} \otimes w_{3}\right\rangle & \operatorname{dim} 1 \\
L_{I, E^{5},-I}=\left\langle u_{0} \otimes w_{3}-I u_{1} \otimes w_{1}\right\rangle & \operatorname{dim} 1 \\
L_{I, E, I}=\left\langle u_{0} \otimes w_{1}+I u_{1} \otimes w_{3}\right\rangle & \operatorname{dim} 1 \\
L_{I, E,-I}=\left\langle u_{0} \otimes w_{3}+I u_{1} \otimes w_{1}\right\rangle & \operatorname{dim} 1
\end{array}\right.
$$




$$
\mathcal{L}_{\overline{2}}=\left\{\begin{array}{rlrl}
L_{-1,1,1}= & \left\langle\left(u_{0} \cdot u_{0}+u_{1} \cdot u_{1}\right) \otimes\left(w_{0} \wedge w_{3}-w_{1} \wedge w_{2}\right),\right. & & \\
& u_{0} \cdot u_{1} \otimes\left(w_{0} \wedge w_{1}-w_{2} \wedge w_{3}\right), & & \\
& \left.\left(u_{0} \cdot u_{0}-u_{1} \cdot u_{1}\right) \otimes\left(w_{0} \wedge w_{3}+w_{1} \wedge w_{2}\right)\right\rangle & \operatorname{dim} 3 \\
L_{-1,1,-1}= & \left\langle\left(u_{0} \cdot u_{0}+u_{1} \cdot u_{1}\right) \otimes\left(w_{0} \wedge w_{1}+w_{2} \wedge w_{3}\right),\right. & \\
& u_{0} \cdot u_{1} \otimes\left(w_{0} \wedge w_{3}+w_{1} \wedge w_{2}\right), & \\
& \left.\left(u_{0} \cdot u_{0}-u_{1} \cdot u_{1}\right) \otimes\left(w_{0} \wedge w_{1}-w_{2} \wedge w_{3}\right)\right\rangle & \operatorname{dim} 3 \\
= & \left\langle\left(u_{0} \cdot u_{0}+u_{1} \cdot u_{1}\right) \otimes\left(w_{1} \wedge w_{3}\right),\right. & \\
& \left.\left(u_{0} \cdot u_{0}-u_{1} \cdot u_{1}\right) \otimes\left(w_{0} \wedge w_{2}\right)\right\rangle & \operatorname{dim} 2 \\
L_{-1, I, 1} & & \operatorname{dim} 1 \\
L_{-1, I,-1}= & \left\langle u_{0} \cdot u_{1} \otimes w_{0} \wedge w_{2}\right\rangle & \\
L_{-1,-1,1}= & \left\langle\left(u_{0} \cdot u_{0}+u_{1} \cdot u_{1}\right) \otimes\left(w_{0} \wedge w_{3}+w_{1} \wedge w_{2}\right),\right. & \\
& u_{0} \cdot u_{1} \otimes\left(w_{0} \wedge w_{1}+w_{2} \wedge w_{3}\right), & \\
& \left.\left(u_{0} \cdot u_{0}-u_{1} \cdot u_{1}\right) \otimes\left(w_{0} \wedge w_{3}-w_{1} \wedge w_{2}\right)\right\rangle & \operatorname{dim} 3 \\
L_{-1,-1,-1}= & \left\langle\left(u_{0} \cdot u_{0}+u_{1} \cdot u_{1}\right) \otimes\left(w_{0} \wedge w_{1}-w_{2} \wedge w_{3}\right),\right. & \\
& u_{0} \cdot u_{1} \otimes\left(w_{0} \wedge w_{3}-w_{1} \wedge w_{2}\right), & \\
& \left.\left(u_{0} \cdot u_{0}-u_{1} \cdot u_{1}\right) \otimes\left(w_{0} \wedge w_{1}+w_{2} \wedge w_{3}\right)\right\rangle & \operatorname{dim} 3 \\
= & \left\langle\left(u_{0} \cdot u_{0}+u_{1} \cdot u_{1}\right) \otimes\left(w_{0} \wedge w_{2}\right),\right. & \operatorname{dim} 2 \\
& \left.\left(u_{0} \cdot u_{0}-u_{1} \cdot u_{1}\right) \otimes\left(w_{1} \wedge w_{3}\right)\right\rangle & \operatorname{dim} 1
\end{array}\right.
$$

and $\mathcal{L}_{\overline{3}}$ dual to $\mathcal{L}_{\overline{1}}$, hence $h_{3}=7, h_{2}=6$ and $h_{1}=19$, and so this is the grading we are looking for.

Along these lines all the gradings can be located by using models of $\mathfrak{f}_{4}$.

\section{Appendix}

Some results which play a fundamental role in our work are included here. Their references may not be so easily accessible and so we state the results (without proof) for the seek of selfcontainedness. So for instance the well known Borel-Serre theorem for Lie groups has a version for algebraic groups which is owed to V. P. Platonov in the following terms:

Theorem 6. ([38, Theorem 3.15, p. 92]) A supersoluble subgroup of semisimple elements of an algebraic group $G$ is contained in the normalizer of a maximal torus.

Here we must recall that a group is called supersolvable (or supersoluble) if it has an invariant normal series whose factors are all cyclic. Any finitely generated abelian group is supersolvable.

Another result that we are applying from the beginning is related to the number of generators of the quasitorus inducing a grading on $\mathfrak{f}_{4}$. By abuse of notation we speak of the number of generators of a quasitorus $Q$ instead of the number of generators of the related finitely generated abelian 
group $\mathfrak{X}(Q)$. When this number is at most 2 we can say for sure that the grading is toral. A first approach to this is the fact that every cyclic grading on $\mathfrak{f}_{4}$ is toral. Indeed, a grading is cyclic if it is induced by a diagonalizable automorphism $f$ of $\mathfrak{F}_{4}$. This is a semisimple element and since $\mathfrak{F}_{4}$ is a connected algebraic group, $f$ is in a maximal torus of $\mathfrak{F}_{4}[10$, Theorem 11.10, p.151]. It is possible to strengthen the previous result to the case in which the grading group has two generators (stated, for instance, in $[2$, Lemma 1.1.3, p. 5]).

Lemma 2. Every subquasitorus $Q$ of $\mathfrak{F}_{4}$ such that $\mathfrak{X}(Q)$ has two generators is toral.

Proof. It is known ([18, Th 3.5.6, p. 93]) that if $G$ is a connected reductive group whose derived subgroup is simply connected, then the centralizer of every semisimple element in $G$ is connected. Let $Q=\overline{\left\langle f_{1}, f_{2}\right\rangle}$ be a (closed) abelian subgroup of semisimple elements in $\mathfrak{F}_{4}$. As any semisimple element belongs to a torus, we replace $f_{1}$ by $t_{1} \in \mathfrak{T}$ by conjugation. Now take $Z=\mathfrak{C}_{\mathfrak{F}_{4}}\left(t_{1}\right)$, which is a connected group. Applying [13, Theorem 1, p. 94] for $n=1$ we finish the proof. But without using this fact, we can go on considering that the element $f_{2} \in Z$ must be in some maximal torus of $Z$, say $T$. But $t_{1}$ is in the center of $Z$ and hence in all the maximal tori of $Z$. We have finished since $\left\langle t_{1}, f_{2}\right\rangle \subset T$, implying $Q \subset T$.

Next we describe the action by conjugation of some elements in $\mathfrak{F}_{4}$ on the automorphisms $g_{1}:=t_{-1,1,-1,1}^{\prime}, g_{2}:=t_{1,-1,-1,1}^{\prime}, g_{3}:=\widetilde{\sigma_{105}}$ and $g_{4}:=t_{1,1,1,-1}^{\prime}$.

\begin{tabular}{|c|c|c|c|}
\hline Element $f$ & $f g_{1} f^{-1}$ & $f g_{2} f^{-1}$ & $f g_{3} f^{-1}$ \\
\hline$\widetilde{\sigma_{94}}$ & $g_{1} g_{2}$ & $g_{2} g_{4}$ & $g_{3}$ \\
$\widetilde{\sigma_{103}}$ & $g_{1} g_{4}$ & $g_{2}$ & $g_{3}$ \\
$\widetilde{\sigma_{468}}$ & $g_{2}$ & $g_{1}$ & $g_{3}$ \\
$\widetilde{\sigma_{485}}$ & $g_{2}$ & $g_{1}$ & $g_{3} g_{4}$ \\
$\widetilde{\sigma_{491}}$ & $g_{1} g_{2}$ & $g_{1} g_{4}$ & $g_{3} g_{4}$ \\
$\psi$ & $g_{1}$ & $g_{3}$ & $g_{2}$ \\
\hline
\end{tabular}

Moreover $f t_{1,1,1, u}^{\prime} f^{-1}=t_{1,1,1, u}^{\prime}$ for any $u \in F^{\times}$and $f$ in the first column on the left of the table. We have used this information to find the nontoral subquasitori of $A(105$, id) in Subsection 5.2.

Note that the indexing of the elements in the Weyl group that we have used from Proposition 3, makes it difficult to see which are these elements for a reader without a computer. In order to help to understand what is going on, we try to describe the representatives as product of generators. Some representatives of the seven order-two orbits are

$$
\left\{ \pm \sigma_{\alpha_{1}}, \pm \sigma_{\alpha_{4}},-1, \sigma_{\alpha_{1}} \sigma_{\alpha_{4}},\left(\sigma_{\alpha_{2}} \sigma_{\alpha_{3}}\right)^{2}\right\}
$$


To be more precise, the reflections related to the 12 long positive roots form exactly the orbit of $\sigma_{55}$ (and $\sigma_{\alpha_{1}}$ could be chosen as a representative), the reflections related to the 12 short positive roots form exactly the orbit of $\sigma_{142}$ (being $\sigma_{\alpha_{4}}$ a representative). By composing two simple reflections, we get a new orbit of order-two elements, that of $\sigma_{28}$, a representative for instance is $\sigma_{\alpha_{1}} \sigma_{\alpha_{4}}$ (or the product of any pair of commuting simple reflections). No new order-two orbits appear when considering products of three simple reflections, but the elements in the $\sigma_{103}$-orbit are compositions of four generators (a representative is $\left.\left(\sigma_{\alpha_{2}} \sigma_{\alpha_{3}}\right)^{2}\right)$. It is known that $-1=\sigma_{405}$ belongs to the Weyl group, it could be realized as $-1=\left(\sigma_{\alpha_{1}} \sigma_{\alpha_{2}} \sigma_{\alpha_{3}} \sigma_{\alpha_{4}}\right)^{6}$. Again all the $-\sigma_{\alpha}$, for a long root $\alpha$, are just the members of the orbit of $\sigma_{42}$, and all the $-\sigma_{\alpha}$, for a short root $\alpha$, exhaust the orbit of $\sigma_{105}$.

And regarding the representatives of the remaining orbits, we will deal only with those providing non-toral gradings. The order-six element $\sigma_{106}$ is in the orbit of $-\sigma_{\alpha_{3}} \sigma_{\alpha_{4}}$. The order-four element $\sigma_{3}$ is in the orbit of $-\sigma_{\alpha_{2}} \sigma_{\alpha_{3}}$. Finally the order-three element $\sigma_{15}$ is in the orbit of $\left(\sigma_{\alpha_{1}} \sigma_{\alpha_{2}} \sigma_{\alpha_{3}} \sigma_{\alpha_{4}}\right)^{4}$.

We described in Subsection 4.2 the way in which any $\sigma \in \mathcal{W}$ could be extended to $\tilde{\sigma} \in \operatorname{aut}\left(\mathfrak{f}_{4}\right)$. However our choice of extensions is not exactly that, for computational reasons. This collection can be downloaded from http://agt2.cie.uma.es/f4bis.zip. In fact, such extension of $\sigma \in \mathcal{W}$ differs from $\tilde{\sigma}$ by an order two element in $\mathfrak{T}$, which is not relevant for our proof.

\section{Thanks.}

We would like to thank Professors Alberto Elduque Palomo for his suggestions on models of exceptional Lie algebras, and Antonio Viruel Arbáizar for his help with the geometric references and also with some results on Lie groups whose transcription to our setting has been relevant for the work. We would like also to thank the referee for accepting the job of refereeing this work.

\section{References}

[1] Adams, J. F.: Lectures on Exceptional Lie Groups. Chicago Lectures in Mathematics. University of Chicago Press, Chicago, IL, 1996.

[2] Andersen, K. K. S.: Some computations for the exceptional groups relevant to the classification of $p$-compact groups. Ph.D. Thesis. Institute for Mathematical Sciences. University of Copenhagen, 2001.

[3] Andersen, K. K. S., Grodal, J., Meeller, J. M. and Viruel, A.: The classification of $p$-compact groups for $p$ odd. Ann. of Math. (2) 167 (2008), no. $1,95-210$. 
[4] Alekseevski, A. V.: Finite commutative Jordan subgroups of simple complex Lie groups. Functional Analysis Appl. 8 (1974), 277-279.

[5] Allison, B., Berman, S., Faulkner, J. and Pianzola, A.: Realization of graded-simple algebras as loop algebras. Forum Math. 20 (2008), no. 3, 395-432.

[6] Bahturin, Y.A. and Shestakov, I. P.: Gradings of simple Jordan algebras and their relation to the gradings of simple associative algebras. Comm. Algebra 29 (2001), no. 9, 4095-4102.

[7] Bahturin, Y. A., Shestakov, I. P. and Zaicev, M. V.: Gradings on simple Jordan and Lie algebras. J. Algebra 283 (2005), no. 2, 849-868.

[8] Bahturin, Y.A. and Tvalavadze, M.: Group gradings on $G_{2}$. Comm. Algebra 37 (2009), no. 3, 885-893.

[9] Bahturin, Y. A. And Zaicev, M. V.: Group gradings on matrix algebras. Canad. Math. Bull. 45 (2002), no. 4, 499-508.

[10] Borel, A.: Linear algebraic Groups (second enlarged edition). Graduate Texts in Mathematics 126. Springer-Verlag, New-York, 1991.

[11] Conway, J. H. And Smith, D. A.: On quaternions and octonions: their geometry, arithmetic, and symmetry. A. K. Peters, Natick, MA, 2003.

[12] Draper, C.: Models of the Lie algebra $F_{4}$. Linear Algebra Appl. 428 (2008), no. 11-12, 2813-2839.

[13] Draper, C. And Martín, C.: Gradings on $\mathfrak{g}_{2}$. Linear Algebra Appl. 418 (2006), no. 1, 85-111.

[14] Draper, C., Martín, C. and Viruel, A.: Fine gradings on the exceptional Lie algebra $\mathfrak{d}_{4}$. To appear in Forum Math.

[15] Elduque, A.: Gradings on octonions. J. Algebra 207 (1998), no. 1, $342-354$.

[16] Elduque, A.: A Lie grading which is not a semigroup grading. Linear Algebra Appl. 418 (2006), no. 1, 312-314.

[17] Fulton, W. And Harris, J.: Representation Theory. A first course. Graduate Texts in Mathematics 129. Springer-Verlag, New York, 1991.

[18] Carter, R. W.: Finite groups of Lie type. Conjugacy classes and complex characters. Pure and Applied Mathematics. A Wiley-Interscience Publication. John Wiley \& Sons., New York, 1985.

[19] GRIESS, R. L.: Elementary abelian p-subgroups of algebraic groups. Geom. Dedicata 39 (1991), no. 3, 253-305.

[20] HavlïC̆ek, M., Patera, J., Pelantová, E. And Tolar, J.: Automorphisms of fine gradings of $\operatorname{sl}(n, \mathbb{C})$ associated with the generalized Pauli matrices. J. Math. Phys. 43 (2002), no. 2, 1083-1094.

[21] Havlï̌̆ek, M., Patera, J. and Pelantová, E.: On Lie gradings II. Linear Algebra Appl. 277 (1998), no. 1-3, 97-125. 
[22] Havlïc̆ek, M., Patera, J. and Pelantová, E.: On Lie gradings III. Gradings of the real forms of classical Lie algebras. Linear Algebra Appl. 314 (2000), no. 1-3, 1-47.

[23] Havlïc̆ek, M., Patera, J., Pelantová, E. and Tolar, J.: On fine gradings and their symmetries. Czechoslovak J. Phys. 51 (2001), no. 4, 383-391.

[24] Hesselink, W. H.: Special and pure gradings of Lie algebras. Math. Z. 179 (1982), no. 1, 135-149.

[25] Humphreys, J. E.: Introduction to Lie algebras and representation theory. Graduate Texts in Mathematics 9. Springer-Verlag, New York-Berlin, 1972.

[26] Humphreys, J. E.: Linear Algebraic Groups. Graduate Texts in Mathematics 21. Springer-Verlag, New York-Heidelberg, 1975.

[27] Jacobson, N.: Lie algebras. Dover Publications, New York, 1979.

[28] Jacobson, N.: Structure and representations of Jordan algebras. Amer. Math. Soc. Colloq. Publ. 39. Amer. Math. Soc., Providence, RI, 1968.

[29] KAC, V. G.: Infinite dimensional Lie algebras. Cambridge University Press, Cambridge, 1990.

[30] Knus, M. A., Merkurjev, A., Rost, M. and Tignol, J. P.: The book of involutions. Amer. Math. Soc. Colloq. Publ. 44. Amer. Math. Soc., Providence, RI, 1998.

[31] Milne, J.S.: Algebraic geometry. Taiaroa Publishing Erehwon, Version 5.00, 2005 (available in http://www.jmilne.org/math/).

[32] De Montigny, M. and Patera, J.: Discrete and continuous graded contractions of Lie algebras and superalgebras. J. Phys. A 24 (1991), no. 3, $525-547$.

[33] Neumann, F.: A theorem of Tits, normalizers of maximal tori and fibrewise Bousfield-Kan completions. Publ. Res. Inst. Math. Sci. 35 (1999), no. $5,711-723$.

[34] Onishchnik, A. L. And Vinberg, E. B.: Lie groups and Lie algebras III Encyclopaedia of Mathematical Sciences 41. Springer-Verlag, Berlin, 1991.

[35] Patera, J., Pelantová, E. And Svobodová, M.: The eight fine gradings of $\operatorname{sl}(4, \mathbb{C})$ and o(6,C). J. Math. Phys. 43 (2002), no. 12, 6353-6378.

[36] Patera, J., Pelantová, E. And Svobodová, M.: Fine gradings of o(4, C). J. Math. Phys. 45 (2004), no. 6, 2188-2198.

[37] Patera, J. and Zassenhaus, H.: On Lie gradings. I. Linear Algebra Appl. 112 (1989), 87-159.

[38] Platonov, V.P.: The theory of algebraic linear groups and periodic groups. American Society Translations (2) 69 (1968), 61-110.

[39] Schafer, R. D.: An introduction to nonassociative algebras. Pure and Applied Mathematics 22. Academic Press, New York-London, 1966. 
[40] Vavpetič, A. And Viruel, A.: On the homotopy type of the classifying space of the exceptional Lie group $F_{4}$. Manuscripta Math. 107 (2002), no. 4, $521-540$.

[41] Vinberg, È. B.: Classification of homogeneous nilpotent elements of a semisimple graded Lie algebra. Trudy Sem. Vektor. Tenzor. Anal. (1979), no. 19, 155-177.

[42] Yoshit, Y.: Coordinate algebras of extended affine Lie algebras of type $A_{1}$. J. Algebra 234 (2000), no. 1, 128-168.

Recibido: 10 de abril de 2007

Revisado: 17 de junio de 2008

Cristina Draper Fontanals Departamento de Matemática Aplicada

ETSI

Campus de Teatinos, s/n 29071, Málaga, Spain cdf@uma.es

Cándido Martín González Departamento de Álgebra, Geometría y Topología Facultad de Ciencias Campus de Teatinos, $\mathrm{s} / \mathrm{n}$ Apdo. 59, 29080 Málaga, Spain. candido@apncs.cie.uma.es

Supported by the Spanish MCYT projects MTM2007-60333 and by the Junta de Andalucía PAI projects FQM-336, FQM-1215 and FQM-2467. 